\title{
Near-BPS baby Skyrmions
}

\author{
Sven Bjarke Gudnason, ${ }^{a}$ Marco Barsanti ${ }^{b, c}$ and Stefano Bolognesi ${ }^{b, c}$ \\ ${ }^{a}$ Institute of Contemporary Mathematics, School of Mathematics and Statistics, Henan University, \\ Kaifeng, Henan 475004, P.R. China \\ ${ }^{b}$ Department of Physics "E. Fermi", University of Pisa, \\ Largo Pontecorvo 3, Ed. C, 56127 Pisa, Italy \\ ${ }^{c}$ INFN, Sezione di Pisa, \\ Largo Pontecorvo 3, Ed. C, 56127 Pisa, Italy \\ E-mail: gudnason@henu.edu.cn, marco.barsanti@phd.unipi.it, \\ stefanobolo@gmail.com
}

ABSTRACT: We consider the baby-Skyrme model in the regime close to the so-called restricted baby-Skyrme model, which is a BPS model with area-preserving diffeomorphism invariance. The perturbation takes the form of the standard kinetic Dirichlet term with a small coefficient $\epsilon$. Classical solutions of this model, to leading order in $\epsilon$, are called restricted harmonic maps. In the BPS limit $(\epsilon \rightarrow 0)$ of the model with the potential being the standard pion-mass term, the solution with unit topological charge is a compacton. Using analytical and numerical arguments we obtain solutions to the problem for topological sectors greater than one. We develop a perturbative scheme in $\epsilon$ with which we can calculate the corrections to the BPS mass. The leading order $\left(\mathcal{O}\left(\epsilon^{1}\right)\right)$ corrections show that the baby Skyrmion with topological charge two is energetically preferred. The binding energy requires us to go to the third order in $\epsilon$ to capture the relevant terms in perturbation theory, however, the binding energy contributes to the total energy at order $\epsilon^{2}$. We find that the baby Skyrmions - in the near-BPS regime - are compactons of topological charge two, that touch each other on their periphery at a single point and with orientations in the attractive channel.

KEywords: Solitons Monopoles and Instantons, Field Theories in Lower Dimensions

ArXiv ePrint: 2006.01726 


\section{Contents}

1 Introduction 1

2 The model 4

2.1 BPS solutions 5

$\begin{array}{lll}2.2 & \text { Energy bound } & 7\end{array}$

3 Perturbation in $\epsilon \quad 8$

3.1 Leading-order correction 8

3.2 Restricted harmonic maps 9

$\begin{array}{lll}3.3 & \mathrm{NLO} \text { and } \mathrm{N}^{2} \mathrm{LO} \text { corrections } & 15\end{array}$

3.4 Axially symmetric solutions 22

4 Numerical calculations $\quad 25$

5 Binding energies $\quad 33$

6 Composite solutions $\quad 46$

$\begin{array}{lll}7 & \text { Conclusion and discussion } & 48\end{array}$

A The leading order energy for various potentials $\quad \mathbf{5 0}$

A.1 $\left(1-\phi^{3}\right)^{s} \quad 50$

A.2 $\left(1-\left(\phi^{3}\right)^{2}\right)^{s} \quad 53$

\section{Introduction}

Nuclear binding energies are relatively small compared to the total nuclear mass, i.e. roughly of the order $1 / 100$ and slightly less than that for the light nuclei. A challenge for solitonic nuclear models, such as the Skyrme model, is to reproduce this result since they usually overestimate the binding energies. This sparked the interest, which has grown in the recent years, in looking for BPS solitonic models and small perturbations around them. In BPS models the energy is proportional to the topological charge, in this case the baryon number, so there is no binding energy between the nuclei. A small perturbation of the BPS model would give, presumably, a small binding energy. These "near-BPS" models have small binding energies already at the classical level. One model of this kind is the so-called BPS Skyrme model which consists of a Lagrangian with a potential plus a sextic term for the pion fields, which is also the squared topological charge density [1-3]. The ordinary Dirichlet (two-derivative kinetic) term and the Skyrme (four-derivative) term are considered as small perturbations. The BPS Skyrme model has the nice phenomenological 
feature of having an infinite-dimensional moduli space for static solutions consisting of all volume-preserving diffeomorphism maps. This resonates well with the liquid drop model of an incompressible fluid so successful in describing nuclear matter. Various theoretical and phenomenological studies have been done regarding the near-BPS Skyrme model [4-8] but a fundamental question still remains unanswered: when the non-BPS perturbations are gradually switched off, the solution in an arbitrary baryonic sector flows to a particular BPS solution, one of many in the infinite dimensional moduli space, but which one? Clearly, it is the infinite number of zero modes - the very same feature which makes the model attractive - that makes the question hard to answer, both analytically and numerically. Attempts to answer this question by direct numerical study have not given a clear definite answer [9]. It was suggested, in modeling the nuclear stars, that the answer could be the axially symmetric multi-Skyrmion [6], but this, for reasons that we will explain, cannot be the true minimum of the energy. It is like searching for a needle in a haystack; clearly we first need a good and well-motivated analytical guess and then we can embark on the numerical study. In this paper we will provide, with the use of a toy model, an analytical guess, at least for a certain class of potentials.

The baby-Skyrme model, as the name suggests, is a toy model for the Skyrme model in one spacetime dimension less, i.e. $2+1$ instead of $3+1$, and with one target-space dimension less, $S^{2}$ instead of $S^{3}$ [10-13]. In this sense it has been used in the past as a test bed for various ideas and conjectures about the more difficult model. Among the various similarities between the two models, both of them possess a BPS restricted model. ${ }^{1}$ For the baby-Skyrme model, the restricted version contains only a four-derivative term (the square of the topological charge) and a potential. This BPS model has area-preserving diffeomorphism invariance for the static solutions and it was actually discovered much earlier than the related model in $3+1$ dimensions [14]. The soliton structure depends very much on the type of potential that is chosen, and in particular on the potential behavior close to the minimum. In this paper we will concentrate on the type of potentials that are quadratic at the minimum and for which the soliton solution of the BPS model is a "compacton" [15-17]. A compacton owe its name to the fact that all nontrivial behavior is contained in a compact region of space, outside of which the field is exactly at the minimum of the potential. The topic of baby Skyrmions is interesting in its own right; particularly, much attention has been paid recently to the applications in magnetic materials, see ref. [18] for a review and refs. [19-21] for some recent theoretical work. So what we describe in this work may hopefully be applied also in these cases, with opportune modifications.

The BPS property of a soliton model is often related to the existence of a supersymmetric extension, but this is not the case for the BPS-Skyrme model in 3+1 dimensions, since its target space is not Kähler [22]. In fact, the first attempt at supersymmetrizing the Skyrme model yielded a supersymmetric model which was in fact more similar to the baby Skyrme model $[23,24]$ in that the target space was effectively $\mathbb{C} P^{1} \sim S^{2}$, although these extensions contain extra terms in the Lagrangian - in particular four time derivatives,

\footnotetext{
${ }^{1}$ We will use the names BPS baby-Skyrme model and restricted baby-Skyrme model indistinguishably in this paper, as often done in the literature.
} 
which are absent in the Skyrme model and the baby Skyrme model. The supersymmetric extension of exactly the baby Skyrme model was constructed later as an $\mathcal{N}=1$ supersymmetric model (having two supercharges) [25, 26]. This model, however, suffered from the fact that the limit in which it would become the restricted baby-Skyrme model does not exist, which was otherwise expected from the BPS property of the purely bosonic version of the baby-Skyrme model. It turns out that extended supersymmetry, namely $\mathcal{N}=2$ supersymmetry (with four supercharges), is necessary for the construction of the BPS baby Skyrme model; a further perhaps surprising fact is that supersymmetry automatically eliminates the kinetic term, indeed yielding the restricted baby-Skyrme model as its bosonic sector [26, 27]. The baby Skyrmion solutions (compactons) turn out to preserve only a quarter of supersymmetry (namely one supercharge) [28, 29]. A successful attempt at constructing a supersymmetric version of the normal Skyrme term in $3+1$ dimensions was made in ref. [30], by enlarging the target space from $\mathrm{SU}(2)$ to $\mathrm{SL}(2, \mathbb{C})$ and this model, like its lower-dimensional cousin, also automatically eliminates the kinetic term. This supersymmetric version of the normal Skyrme model (without the kinetic term) does contain soliton solutions, but they are not BPS [31].

Deformations of BPS models by small perturbations have been discussed in various cases, see for example refs. $[26,32]$. The general feature is that the moduli space of solutions is lifted by some effective potential and the solutions, when the perturbation is small, lie close to the minima of said potential. In the case of BPS models with volume preserving diffeomorphism symmetry, the moduli space is infinite dimensional and thus the problem becomes considerably more difficult. A rigorous mathematical definition of the problem has been put forward in ref. [33]. In particular, if the perturbation is the kinetic Dirichlet term, solutions have been coined "restricted harmonic". Harmonic because they minimize the Dirichlet term, restricted because the minimization is constrained to a sub-space of the functional space, namely the infinite-dimensional moduli space of solutions of the BPS equations. In this paper, we will show that the concept of restricted harmonicity is still not enough to solve the problem in the case of compactons. In fact, for compactons, the restricted harmonic maps are still an infinite-dimensional subspace of the moduli space. Finding the correct solution thus requires to go to even higher order in the perturbation. This in particular affects the way the binding energy is going to zero as the perturbation is switched off.

In this paper, we focus on the baby-Skyrme model with a standard pion mass term and the perturbation is only the Dirichlet (kinetic) term with coefficient $\epsilon$. When $\epsilon$ is switched off, the restricted baby-Skyrme model is recovered. We address the near BPS behavior of the solutions in the model with topological charges $Q=1,2$ and 4 by large brute-force numerical simulations and by developing a framework for perturbation theory around the BPS solution. We find that the correction to the energy (mass) of the axially symmetric compactons, to leading order in $\epsilon$, comes from the kinetic (Dirichlet) term and remains inside the compacton domain. This is specific to the case of the compactons. This leadingorder correction thus cannot teach us anything about the binding energy of the solutions in the near-BPS regime. A main result of this paper, in the part of the perturbation theory, is that the binding energy does not appear at any particular order, if we strictly truncate 
the correction to a specific polynomial order. Nevertheless, we can calculate the binding energy using a linearized perturbation and it shows that the contribution to the binding energy is in some sense all-order, which we could choose to interpret as a "nonperturbative effect". This happens because the perturbation that gives rise to the binding energy takes the form $\exp (-r / \sqrt{\epsilon})$ and thus does not have any nonvanishing Taylor coefficients upon expansion. For the choice of potential we work with in this paper, we find that the nearBPS solutions take the form of charge-2 baby Skyrmions placed very close to each other so that their periphery just touch.

The paper is organized as follows. In section 2 we give a brief review of the baby-Skyrme model and of the solutions in the BPS limit. In section 3 we develop a systematic framework for calculating the perturbations to the BPS soliton, which describes the solutions in the regime near the restricted BPS model with the Dirichlet term being a small perturbation. In order to calculate the binding energy, we find that it is necessary to go to the third order or the next-to-next-to-leading order in perturbation theory. We test the perturbative scheme on axially symmetric compactons, for which we have a comparison with the exact numerical solutions. In section 4 we present the results of the full numerical PDE computations for topological charges $Q=1,2,4$ and calculate the total energy to be used for comparison with the results in section 5, where we calculate the binding energies in perturbation theory, by solving a linear PDE. In section 6 we contemplate what the solutions with large topological charges look like. Finally we conclude with a discussion in section 7 . This paper studies primarily the standard pion mass term, but the cases of other potentials are relegated to appendix A.

\section{The model}

The model is based on the BPS baby-Skyrme model with a non-BPS deformation which is manifested as the kinetic term with coefficient $\epsilon$

$$
\mathcal{L}[\phi]=\epsilon \mathcal{L}_{2}[\phi]+\mathcal{L}_{4}[\phi]-V(\phi)+\frac{1}{2} \lambda(\phi \cdot \phi-1),
$$

where the kinetic term and the Skyrme terms, respectively, are given by

$$
\begin{aligned}
& \mathcal{L}_{2}[\boldsymbol{\phi}]=-\frac{1}{2}\left(\partial_{\mu} \boldsymbol{\phi} \cdot \partial^{\mu} \boldsymbol{\phi}\right), \\
& \mathcal{L}_{4}[\boldsymbol{\phi}]=-\frac{1}{4}\left(\partial_{\mu} \boldsymbol{\phi} \cdot \partial^{\mu} \boldsymbol{\phi}\right)\left(\partial_{\nu} \boldsymbol{\phi} \cdot \partial^{\nu} \boldsymbol{\phi}\right)+\frac{1}{4}\left(\partial_{\mu} \boldsymbol{\phi} \cdot \partial_{\nu} \boldsymbol{\phi}\right)\left(\partial^{\mu} \boldsymbol{\phi} \cdot \partial^{\nu} \boldsymbol{\phi}\right),
\end{aligned}
$$

and $\phi=\left(\phi^{1}, \phi^{2}, \phi^{3}\right)$ is a real 3 -vector on which the nonlinear sigma model constraint $\phi \cdot \phi=1$ is imposed by the Lagrange multiplier, $\lambda$, and the metric signature is here taken to be the mostly positive one. The symmetry of the Lagrangian for $V=0$ is $\tilde{G}=\mathrm{O}(3)$. In the vacuum, this symmetry is spontaneously broken to $\tilde{H} \simeq \mathrm{O}(2)$, which gives rise to the target space $\tilde{G} / \tilde{H} \simeq \mathrm{O}(3) / \mathrm{O}(2) \simeq S^{2}$.

If we choose the potential of the form

$$
V(\phi)=\frac{1}{p} m^{2}\left(1-\phi^{3}\right)^{p}
$$


with $\frac{1}{2} \leq p<2$, then the soliton solution, called a baby Skyrmion, has support on a compact domain in the limit of $\epsilon \rightarrow 0$ and is then dubbed a compacton [16]. In this limit the model is then called the BPS baby-Skyrme model or restricted baby-Skyrme model.

In this paper, we will use the pion mass term

$$
V(\phi)=m^{2}\left(1-\phi^{3}\right)
$$

which turns the baby Skyrmion into a compacton in the limit $\epsilon \rightarrow 0$.

The topological degree of the baby Skyrmion or compacton is given by

$$
Q=\frac{1}{4 \pi} \int \mathrm{d}^{2} x \mathcal{Q}_{12}=-\frac{1}{4 \pi} \int \mathrm{d}^{2} x \boldsymbol{\phi} \cdot \partial_{1} \phi \times \partial_{2} \phi
$$

which also counts the number of baby Skyrmions in $\mathbb{R}^{2}$.

For the analytic calculations, it will prove convenient to use the following parametrization

$$
\phi=\frac{1}{1+|\omega|^{2}}\left(\begin{array}{c}
\omega+\bar{\omega} \\
-i(\omega-\bar{\omega}) \\
1-|\omega|^{2}
\end{array}\right)
$$

in terms of which the nonlinear sigma model constraint $\phi \cdot \phi=1$ is manifest and we can thus write the Lagrangian components as

$$
\begin{aligned}
& \mathcal{L}_{2}[\omega, \bar{\omega}]=-2 \frac{\partial_{\mu} \omega \partial^{\mu} \bar{\omega}}{\left(1+|\omega|^{2}\right)^{2}} \\
& \mathcal{L}_{4}[\omega, \bar{\omega}]=-2 \frac{\left(\partial_{\mu} \omega \partial^{\mu} \bar{\omega}\right)\left(\partial_{\nu} \omega \partial^{\nu} \bar{\omega}\right)-\left(\partial_{\mu} \omega \partial_{\nu} \bar{\omega}\right)\left(\partial^{\mu} \omega \partial^{\nu} \bar{\omega}\right)}{\left(1+|\omega|^{2}\right)^{4}}
\end{aligned}
$$

and the potential as

$$
V(\omega, \bar{\omega})=2 m^{2} \frac{|\omega|^{2}}{1+|\omega|^{2}} .
$$

In this parametrization, the topological charge reads

$$
Q=-\frac{\mathrm{i}}{2 \pi} \int \mathrm{d}^{2} x \frac{\epsilon^{i j} \partial_{i} \omega \partial_{j} \bar{\omega}}{\left(1+|\omega|^{2}\right)^{2}}
$$

We will keep both formalisms in this paper, as $\omega$ is useful for analytical calculations and $\phi$ is useful for the numerical analysis.

\section{$2.1 \quad$ BPS solutions}

The model contains a BPS submodel, which is given by setting $\epsilon=0$ in the Lagrangian (2.1):

$$
\mathcal{L}^{\mathrm{BPS}}[\phi]=\mathcal{L}_{4}[\phi]-V(\phi)+\lambda(\phi \cdot \phi-1) .
$$

In order to find the BPS equation and corresponding solutions for each topological sector, $Q$, it proves convenient to rewrite the static energy density (ignoring the Lagrange 
multiplier term)

$$
\begin{aligned}
\mathcal{E}^{\mathrm{BPS}}[\boldsymbol{\phi}] & =\frac{1}{4}\left(\partial_{i} \boldsymbol{\phi} \cdot \partial_{i} \phi\right)\left(\partial_{j} \phi \cdot \partial_{j} \phi\right)-\frac{1}{4}\left(\partial_{i} \phi \cdot \partial_{j} \phi\right)\left(\partial_{i} \phi \cdot \partial_{j} \phi\right)+m^{2}\left(1-\phi^{3}\right) \\
& =\frac{1}{4}\left(\partial_{i} \phi \times \partial_{j} \phi\right) \cdot\left(\partial_{i} \phi \times \partial_{j} \phi\right)+m^{2}\left(1-\phi^{3}\right) \\
& =\frac{1}{4}\left(\phi \cdot \partial_{i} \phi \times \partial_{j} \phi\right)\left(\phi \cdot \partial_{i} \phi \times \partial_{j} \phi\right)+m^{2}\left(1-\phi^{3}\right) \\
& =\frac{1}{2}\left(\phi \cdot \partial_{1} \phi \times \partial_{2} \phi\right)\left(\phi \cdot \partial_{1} \phi \times \partial_{2} \phi\right)+m^{2}\left(1-\phi^{3}\right) \\
& =\frac{1}{2}\left[\left(\phi \cdot \partial_{1} \phi \times \partial_{2} \phi\right) \mp m \sqrt{2\left(1-\phi^{3}\right)}\right]^{2} \pm m \sqrt{2\left(1-\phi^{3}\right)}\left(\phi \cdot \partial_{1} \phi \times \partial_{2} \phi\right),
\end{aligned}
$$

where we have performed a Bogomol'nyi completion in the last step. The last line of the above equation contains the square of a real expression (the BPS equation) and a boundary term, which we will show shortly is proportional to the topological charge. Setting the expression in the square equal to zero yields the BPS equation, which thus reads:

$$
\mathcal{Q}_{12}=-\phi \cdot \partial_{1} \phi \times \partial_{2} \phi=\mp m \sqrt{2\left(1-\phi^{3}\right)},
$$

and when satisfied, the total energy is proportional to the topological degree of the baby Skyrmion, $Q$.

Changing parametrization of $\phi$ to stereographic coordinates (2.7), the BPS equation now reads

$$
\frac{\partial_{1} \omega \partial_{2} \bar{\omega}-\partial_{2} \omega \partial_{1} \bar{\omega}}{\left(1+|\omega|^{2}\right)^{2}}=\frac{\partial_{r} \omega \partial_{\theta} \bar{\omega}-\partial_{\theta} \omega \partial_{r} \bar{\omega}}{r\left(1+|\omega|^{2}\right)^{2}}=\mp \mathrm{i} m \sqrt{\frac{|\omega|^{2}}{1+|\omega|^{2}}} .
$$

Inserting the Ansatz $\omega=e^{\mathrm{i} N \theta} \zeta(r)$, we get

$$
\frac{\partial_{r} \zeta}{r}= \pm \frac{m}{2 N}\left(1+\zeta^{2}\right)^{\frac{3}{2}}
$$

which we can write as

$$
\int \frac{\mathrm{d} \zeta}{\left(1+\zeta^{2}\right)^{\frac{3}{2}}}=\frac{\zeta}{\sqrt{1+\zeta^{2}}}= \pm \frac{m}{2 N} \int \mathrm{d} r r= \pm \frac{m}{4 N} r^{2} \pm \kappa= \pm\left(\xi^{2}+\kappa\right) .
$$

Choosing the lower sign, we get

$$
\frac{\zeta}{\sqrt{1+\zeta^{2}}}=-\xi^{2}-\kappa
$$

which with $\kappa=-\xi_{0}^{2}$ can be written as

$$
\zeta=\frac{\xi_{0}^{2}-\xi^{2}}{\sqrt{\left(1-\xi_{0}^{2}+\xi^{2}\right)\left(1+\xi_{0}^{2}-\xi^{2}\right)}} .
$$

If we set the integration constant $\xi_{0}=1$, we can move the coordinate singularity to $\xi=0$, obtaining the solution

$$
\zeta=\frac{1-\xi^{2}}{\xi \sqrt{2-\xi^{2}}}, \quad \xi \in[0,1]
$$


where $\xi \equiv \frac{r}{R}$ and the compacton radius is

$$
R=\sqrt{\frac{4 N}{m}} .
$$

It will prove useful to calculate the BPS mass which is simply the boundary term of eq. (2.13):

$$
\begin{aligned}
M^{\mathrm{BPS}} & =\int \mathrm{d}^{2} x \mathcal{E}^{\mathrm{BPS}}[\boldsymbol{\phi}] \\
& = \pm \mathrm{i} 4 m \int \mathrm{d}^{2} x \frac{|\omega|}{\left(1+|\omega|^{2}\right)^{\frac{5}{2}}} \epsilon^{i j} \partial_{i} \omega \partial_{j} \bar{\omega} \\
& = \pm 16 \pi m N \int \mathrm{d} r \frac{\zeta^{2}}{\left(1+\zeta^{2}\right)^{\frac{5}{2}}} \partial_{r} \zeta \\
& =-16 \pi m N \int_{\infty}^{0} \mathrm{~d} \zeta \frac{\zeta^{2}}{\left(1+\zeta^{2}\right)^{\frac{5}{2}}} \\
& =\frac{16 \pi m N}{3} .
\end{aligned}
$$

On the third line above, we see that the entire expression is indeed a boundary term, as promised, and it can be written as an integral over the target space coordinate $\zeta$ (fourth line). In the fourth line we have chosen the lower sign, corresponding to the boundary conditions $\zeta(0) \rightarrow \infty$ and $\zeta(R)=0$.

Note that the topological charge of this axially symmetric configuration is

$$
Q=-2 N \int \mathrm{d} r \frac{\zeta \partial_{r} \zeta}{\left(1+\zeta^{2}\right)^{2}}=N
$$

For axially symmetric baby Skyrmions, we will use $N$ (which is equal to $Q$ ) to denote the topological charge, whereas for more complicated configurations, $Q$ is the total topological charge. For instance, later it will be useful to consider a $Q=4$ baby Skyrmion that is composed by two $N=2$ axially symmetric solutions.

\section{$2.2 \quad$ Energy bound}

In the previous section, we have shown that the energy is bounded from below by the Bogomol'nyi type bound for the BPS sector $\epsilon=0$,

$$
E_{4+0} \geq M^{\mathrm{BPS}}=\frac{16 \pi m|Q|}{3} .
$$


In addition, it can also be shown that there is a bound in the energy for the first term in the Lagrangian (2.1),

$$
\begin{aligned}
E_{2} & =2 \epsilon \int \mathrm{d}^{2} x \frac{\partial_{i} \omega \partial_{i} \bar{\omega}}{\left(1+|\omega|^{2}\right)^{2}} \\
& =4 \epsilon \int \mathrm{d}^{2} x \frac{|\partial \omega|^{2}+|\bar{\partial} \omega|^{2}}{\left(1+|\omega|^{2}\right)^{2}} \\
& \geq 4 \epsilon\left|\int \mathrm{d}^{2} x \frac{|\partial \omega|^{2}-|\bar{\partial} \omega|^{2}}{\left(1+|\omega|^{2}\right)^{2}}\right| \\
& \geq 2 \epsilon\left|-\mathrm{i} \int \mathrm{d}^{2} x \frac{\epsilon^{i j} \partial_{i} \omega \partial_{j} \bar{\omega}}{\left(1+|\omega|^{2}\right)^{2}}\right| \\
& \geq 4 \pi \epsilon|Q|=M^{\text {lump }} .
\end{aligned}
$$

The total energy in the model (2.1) is thus bounded from below by

$$
\begin{aligned}
E_{2+4+0} & \geq M^{\mathrm{BPS}}+M^{\mathrm{lump}} \\
& \geq 16 \pi|Q|\left(\frac{\epsilon}{4}+\frac{m}{3}\right) .
\end{aligned}
$$

This bound is, however, only satisfied in the two limits: $\epsilon \rightarrow 0$ (BPS baby-Skyrmion limit) and $\epsilon \rightarrow \infty$ (BPS lump limit) [26].

\section{Perturbation in $\epsilon$}

We will now consider making a perturbation in $\epsilon$ around a background solution. That is, the background, $\boldsymbol{\varphi}$, is a BPS compacton, which minimizes the BPS submodel (2.12):

$$
\delta \mathcal{L}^{\mathrm{BPS}}[\varphi]=0 .
$$

For reference, it will be useful to write the explicit form of the charge- $N$ axially symmetric compacton solution (2.20) in the vector coordinate $\boldsymbol{\varphi}$ :

$$
\boldsymbol{\varphi}=\left(\begin{array}{c}
\sin f(r) \cos (N \theta-\alpha) \\
\sin f(r) \sin (N \theta-\alpha) \\
\cos f(r)
\end{array}\right), \quad f(r)=\arccos \left(-1+\frac{4 r^{2}}{R^{2}}-\frac{2 r^{4}}{R^{4}}\right)
$$

with $R$ the compacton radius $(2.21)$ and $x+\mathrm{i} y=r e^{\mathrm{i} \theta}$ the standard polar coordinates in $\mathbb{R}^{2}$.

We will consider the corrections to the energy order by order in the following sections.

\subsection{Leading-order correction}

The leading order correction, proportional to $\epsilon$, comes from inserting the background solution into the kinetic term. However, since $\varphi$ depends on the moduli parameters of the BPS sector, not all the possible background solutions are equivalent choices at this order. Recently, it has been conjectured that the right choice among all the possible maps is given 
by the minimizer of $E_{2}$ on the moduli space of the BPS solutions [26, 33]. A map respecting this request is said to be restricted harmonic and, in the case of a single compacton, it is identified by the axially symmetric solution (2.20). The complete proof of this statement is given in the next section.

The first order correction in terms of $\epsilon$ is therefore

$$
\begin{aligned}
\epsilon M^{\mathrm{LO}}(N) & =-\epsilon \int \mathrm{d}^{2} x \mathcal{L}_{2}[\boldsymbol{\varphi}] \\
& =4 \pi \epsilon \int \mathrm{d} r \frac{r \zeta_{r}^{2}+\frac{N^{2}}{r} \zeta^{2}}{\left(1+\zeta^{2}\right)^{2}} \\
& =4 \pi \epsilon \int \mathrm{d} \xi \frac{\xi \zeta_{\xi}^{2}+\frac{N^{2}}{\xi} \zeta^{2}}{\left(1+\zeta^{2}\right)^{2}} \\
& =4 \pi \epsilon\left(2 \log 2+\frac{7 N^{2}}{24}\right) .
\end{aligned}
$$

The parenthesis on the last line takes the value 1.678 for $N=1$, which is about $5 / 3$ of the energy bound for the kinetic term.

To this order, the energy reads

$$
\begin{aligned}
E(\epsilon, N) & =M^{\mathrm{BPS}}(N)+\epsilon M^{\mathrm{LO}}(N) \\
& =\frac{16 \pi m N}{3}+8 \pi \epsilon \log 2+\frac{7 \pi \epsilon N^{2}}{6} .
\end{aligned}
$$

We can now consider the energy per $N$ as a function of $N$. In particular, this function has a minimum

$$
\frac{\mathrm{d}}{\mathrm{d} N}\left(\frac{E(\epsilon, N)}{N}\right)=-\frac{8 \pi \epsilon \log 2}{N^{2}}+\frac{7 \pi \epsilon}{6}=0,
$$

which is

$$
N_{\star}=4 \sqrt{\frac{3 \log 2}{7}} \simeq 2.180 .
$$

This means that for an axially symmetric configuration with topological charge $N=1,2$, the leading-order energy (i.e. to $\mathcal{O}(\epsilon)$ ) per charge, $Q=N$, decreases as a function of $N$. This implies that an axially symmetric 2-Skyrmion has lower energy than 2 well separated 1-Skyrmions. ${ }^{2}$

This, in particular, means that for parametrically small $\epsilon$, the lowest energy configuration with topological charge $Q=2 M$ will consist of $M$ 2-Skyrmions at a separation distance that is not determined at this order in the calculation.

\subsection{Restricted harmonic maps}

In the previous section, the notion of restricted harmonic maps [33] was briefly discussed. In what follows, we will formally define such a map that is used as the zeroth-order background

\footnotetext{
${ }^{2}$ This happens also for large values of $\epsilon=\mathcal{O}(1)$, where the charge $Q$ solutions are composed by two $N=2$ solutions as end caps, sandwiching $(Q-4) / 2$ stretched $N=2$ solutions for even $Q$ and $(Q-5) / 2$ stretched $N=2$ solutions with an extra squashed $N=1$ solution [34]. In ref. [34] they have been coined baby-Skyrmion chains.
} 
of a field expansion in a near-BPS model. After the general definition, we focus on the nearBPS baby-Skyrme model, giving a review of the criterion obtained in ref. [33] to identify the correct restricted harmonic map. The axially symmetric solution used in eq. (3.3) is verified to respect this criterium. In the last part of this section, we further verify that even two axially symmetric compactons sitting side-by-side with a random orientation are restricted harmonic. The last result will be useful in section 5 in which we discuss the interaction between two near-BPS baby Skyrmions and calculate the binding energy.

Given a set of scalar fields $\phi^{a}$ mapping $d+1$ dimensional Minkowski spacetime $(\mathcal{M}, \eta)$ with flat metric $\eta$ to the target space $(\mathcal{N}, h)$ with $\mathcal{N}=S^{2}$ being the 2 -sphere with metric $h$, a near-BPS Lagrangian $\mathcal{L}$ can be written as

$$
\mathcal{L}=\mathcal{L}^{\mathrm{BPS}}+\epsilon \mathcal{L}_{2}
$$

where $\mathcal{L}^{\text {BPS }}$ describes the pure BPS sector and $\epsilon$ is a small parameter multiplying the Dirichlet term

$$
\mathcal{L}_{2}=-\frac{1}{2} h_{a b} \partial_{\mu} \phi^{a} \partial^{\mu} \phi^{b},
$$

with $h_{a b}$ representing the metric of the target manifold $\mathcal{N}$.

Taking into account only the BPS sector, we denote by $\varphi^{a}(x, \lambda)$ the generic static solution of the model that depends on the moduli parameters $\lambda$. Explicitly, we consider a BPS system for which $\lambda$ consists of all the possible orbits in the group of volume-preserving diffeomorphisms.

In the limit of $\epsilon \rightarrow 0$, the total static energy $E$ of the system (3.7), at the lowest order in $\epsilon$, takes the form

$$
E=E^{\mathrm{BPS}}\left[\varphi^{a}\left(x, \lambda^{*}\right)\right]+\epsilon E_{2}\left[\varphi^{a}\left(x, \lambda^{*}\right)\right],
$$

where $\lambda^{*} \subset \lambda$ is the subset of $\lambda$ that minimizes the Dirichlet energy $E_{2}$

$$
E_{2}=\frac{1}{2} \int_{\mathcal{M}} \mathrm{d}^{d} x h_{a b} \partial_{i} \phi^{a} \partial_{i} \phi^{b} .
$$

The map $\varphi^{a}\left(x, \lambda^{*}\right)$ that locally minimizes $E_{2}$ within the domain of the group of volumepreserving diffeomorphisms is said to be restricted harmonic.

A possible proof for the expression (3.9) can be performed assuming a Taylor expansion for the field $\phi$ in terms of $\epsilon$,

$$
\phi=\phi^{(0)}+\epsilon \phi^{(1)}+\epsilon^{2} \phi^{(2)}+\cdots
$$

All orders of $\phi$ can be solved by minimizing the total action $S$ order by order. It is important to note that a perturbative expansion in terms of $\epsilon$ is not always possible for the exact static solution $\phi$, since the dependence on such a parameter could be nonanalytic. Although the leading order energy (i.e. to order $\mathcal{O}(\epsilon)$ ) does take the form of eq. (3.9), it turns out that higher-order contributions (of order $\mathcal{O}\left(\epsilon^{2}\right)$ and beyond) include a nonanalytic contribution to the energy which captures the binding energy, see the next section. We therefore assume this hypothesis here only to give a simple justification of the expression (3.9); for a more complete treatment of the topic, see refs. [26, 33]. 
Since we are dealing only with static configurations, we consider the static energy $E$ to be minimized instead of the action $S$ and we analogously write a Taylor series for the energy

$$
E=E^{(0)}+\epsilon E^{(1)}+\epsilon^{2} E^{(2)}+\cdots
$$

Hence, the zeroth-order of the static energy is given by

$$
E^{(0)}=-\int_{\mathcal{M}} \mathrm{d}^{d} x \mathcal{L}^{\mathrm{BPS}}\left(\phi^{(0)}\right),
$$

whose static solution is by definition

$$
\phi^{(0)}=\varphi^{a}(x, \lambda) .
$$

The first-order in the expansion reads

$$
\begin{aligned}
\epsilon E^{(1)} & =-\int_{\mathcal{M}} \mathrm{d}^{d} x\left[\epsilon \phi^{(1)}\left(\left.\frac{\partial \mathcal{L}^{\mathrm{BPS}}}{\partial \phi}\right|_{\phi^{(0)}}-\left.\partial_{i}\left(\frac{\partial \mathcal{L}^{\mathrm{BPS}}}{\partial \partial_{i} \phi}\right)\right|_{\phi^{(0)}}\right)+\epsilon \mathcal{L}_{2}\left(\phi^{(0)}\right)\right] \\
& =-\int_{\mathcal{M}} \mathrm{d}^{d} x \epsilon \mathcal{L}_{2}\left(\phi^{(0)}\right),
\end{aligned}
$$

where the first term in the parenthesis on the first line vanishes due to the equations of motion. Since the functional $E^{(1)}$ contains only the background solution, the minimum of $E^{(1)}$ must be sought not among all the field configurations but only within the moduli space of the BPS solution (3.14). Therefore, following the principle of least energy (action)

$$
\delta E^{(1)}=0 \Rightarrow \frac{\partial E_{2}\left[\varphi^{a}(x, \lambda)\right]}{\partial \lambda}=0,
$$

whose solution $\varphi^{a}\left(x, \lambda^{*}\right)$ is by definition the restricted harmonic map discussed above. We clarify that for an infinite moduli space, such as the group of volume-preserving diffeomorphism, the derivative with respect to $\lambda$ does not have a mathematically well-defined meaning and we use it here only to simplify the notation. Summing the zeroth-order (3.13) and the first order (3.15) of the energy calculated with the solution $\varphi^{a}\left(x, \lambda^{*}\right)$ we obtain the expression (3.9), as we wanted to prove.

After the formal definition, we need some practical tools to calculate the restricted harmonic maps of a given system. A mathematical criterion that is able to identify whether a map is restricted harmonic (or not) has been proposed in ref. [33]. In the following, we review the theorem given there, which we use for the near-BPS baby-Skyrme model in the case of a single and two compactons.

Given a smooth map $\phi$ from the manifold $\mathcal{M}$ with metric $g=g_{i j} \mathrm{~d} x^{i} \otimes \mathrm{d} x^{j}$ to the manifold $\mathcal{N}$ with metric $h=h_{a b} \mathrm{~d} \phi^{a} \otimes \mathrm{d} \phi^{b}$, the Dirichlet energy is defined as

$$
E_{2}=\int_{\mathcal{M}} \frac{1}{2} \mathrm{~d}^{d} x h_{a b} g^{i j} \partial_{i} \phi^{a} \partial_{j} \phi^{b},
$$

where $d$ is the number of dimensions of the space $\mathcal{M}$. Using the map $\phi$ we can construct the pull-back $\phi^{*} h$ of the metric $h$ to $\mathcal{M}$

$$
\phi^{*} h=h_{a b} \frac{\partial \phi^{a}}{\partial x^{i}} \frac{\partial \phi^{b}}{\partial x^{j}} \mathrm{~d} x^{i} \otimes \mathrm{d} x^{j}
$$


which is necessary for what follows. Among all the maps $\phi$ with finite Dirichlet energy connected by a volume-preserving diffeomorphisms, a map $\tilde{\phi}$ is restricted harmonic if and only if the one-form $\operatorname{div} \tilde{\phi}^{*} h$ on $\mathcal{M}$ is exact [33]. It is useful to recall that the divergence of a symmetric $(0,2)$ tensor $\omega=\omega_{i j} \mathrm{~d} x^{i} \otimes \mathrm{d} x^{j}$ on $\mathcal{M}$ it is nothing but

$$
\operatorname{div} \omega=D^{i} \omega_{i j} \mathrm{~d} x^{j}=g^{i k}\left(\partial_{k} \omega_{i j}-\Gamma_{k i}^{l} \omega_{l j}-\Gamma_{k j}^{l} \omega_{i l}\right) \mathrm{d} x^{j},
$$

with the connection $\Gamma$ (Christoffel symbols) defined as

$$
\Gamma_{j k}^{i}=\frac{1}{2} g^{i l}\left(\frac{\partial g_{l j}}{\partial x^{k}}+\frac{\partial g_{l k}}{\partial x^{j}}-\frac{\partial g_{j k}}{\partial x^{l}}\right)
$$

Using this theorem we verify that the axially symmetric solution used in eq. (3.3) is restricted harmonic. Using polar coordinates on $\mathcal{M}=\mathbb{R}^{2}$ and the vector notation $\phi^{a}$ for the field, with the constraint $\phi^{a} \phi^{a}=1$, we write a generic $Q=N$ axially symmetric compacton in the form

$$
\phi^{a}=\left(\begin{array}{c}
\sin f(r) \cos (N \theta-\alpha) \\
\sin f(r) \sin (N \theta-\alpha) \\
\cos f(r)
\end{array}\right),
$$

where the function $f$ depends only on the radial coordinate and $\alpha$ is the orientation phase [14]. With this choice, the metric $h$ reduces to the standard euclidean metric and the pull-back $\phi^{*} h$ of $h$ can be written as

$$
\phi^{*} h=\frac{\partial \phi^{a}}{\partial \tilde{x}^{i}} \frac{\partial \phi^{a}}{\partial \tilde{x}^{j}} \mathrm{~d} \tilde{x}^{i} \otimes \mathrm{d} \tilde{x}^{j}=\left(f^{\prime}\right)^{2} \mathrm{~d} r^{2}+N^{2}(\sin f)^{2} \mathrm{~d} \theta^{2},
$$

with $\mathrm{d} \tilde{x}^{i}=(\mathrm{d} r, \mathrm{~d} \theta)$. Note that there is no longer any dependence on the orientation phase $\alpha$. Taking the divergence of this tensor yields

$$
\begin{aligned}
\operatorname{div} \phi^{*} h & =\tilde{D}^{i} \tilde{\partial}_{i} \phi^{a} \tilde{\partial}_{j} \phi^{a} \mathrm{~d} \tilde{x}^{j} \\
& =\tilde{g}^{i k}\left(\tilde{\partial}_{k}\left(\tilde{\partial}_{i} \phi^{a} \tilde{\partial}_{j} \phi^{a}\right)-\tilde{\Gamma}_{k i}^{l} \tilde{\partial}_{l} \phi^{a} \tilde{\partial}_{j} \phi^{a}-\tilde{\Gamma}_{k j}^{l} \tilde{\partial}_{i} \phi^{a} \tilde{\partial}_{l} \phi^{a}\right) \mathrm{d} \tilde{x}^{j} \\
& =\left(2 f^{\prime} f^{\prime \prime}+\frac{\left(f^{\prime}\right)^{2}}{r}-\frac{N^{2} \sin ^{2} f}{r^{3}}\right) \mathrm{d} r \\
& \equiv \omega_{i} \mathrm{~d} \tilde{x}^{i},
\end{aligned}
$$

resulting in a one-form whose exactness we must prove. Here, it is useful to recall that according to Poincaré's lemma all closed forms on a contractible manifold are exact. Hence, to complete the proof we must verify that $\mathrm{d}\left(\operatorname{div} \phi^{*} h\right)=0$, with $\mathrm{d}$ being the exterior derivative. Explicitly,

$$
\begin{aligned}
\mathrm{d}\left(\operatorname{div} \phi^{*} h\right)=\mathrm{d} \omega & =\left(\tilde{\partial}_{i} \omega_{j}-\tilde{\partial}_{j} \omega_{i}\right) \mathrm{d} \tilde{x}^{i} \wedge \mathrm{d} \tilde{x}^{j} \\
& =2\left(\partial_{r} \omega_{\theta}-\partial_{\theta} \omega_{r}\right) \mathrm{d} r \wedge \mathrm{d} \theta \\
& =0
\end{aligned}
$$

which gives the necessary and sufficient condition for eq. (3.21) to be restricted harmonic. 
The same proof can be extended to the case of two axially symmetric compactons sitting side-by-side without overlap. A solution for two separated compactons with $Q=$ $N+N$ and random orientations can be written as

$$
\phi^{a}= \begin{cases}\phi_{1}^{a}\left(x+x_{0}, y\right) & x<0 \\ \phi_{2}^{a}\left(x-x_{0}, y\right) & x \geq 0\end{cases}
$$

where $\phi_{1}^{a}, \phi_{2}^{a}$ are axially symmetric with respect to the points $\left(-x_{0}, 0\right)$ and $\left(x_{0}, 0\right)$ respectively, i.e.,

$$
\phi_{1}^{a}=\left(\begin{array}{c}
\sin f\left(r_{+}\right) \cos \left(N \theta_{+}-\alpha\right) \\
\sin f\left(r_{+}\right) \sin \left(N \theta_{+}-\alpha\right) \\
\cos f\left(r_{+}\right)
\end{array}\right), \quad \phi_{2}^{a}=\left(\begin{array}{c}
\sin f\left(r_{-}\right) \cos \left(N \theta_{-}-\beta\right) \\
\sin f\left(r_{-}\right) \sin \left(N \theta_{-}-\beta\right) \\
\cos f\left(r_{-}\right)
\end{array}\right),
$$

with

$$
\begin{array}{ll}
r_{+}=\sqrt{\left(x+x_{0}\right)^{2}+y^{2}}, & r_{-}=\sqrt{\left(x-x_{0}\right)^{2}+y^{2}}, \\
\theta_{+}=\arctan \left(\frac{y}{x+x_{0}}\right), & \theta_{-}=\arctan \left(\frac{y}{x-x_{0}}\right),
\end{array}
$$

and $\alpha, \beta$ are two independent phases. Note that in order to have two separated compactons we require $\left|x_{0}\right|>R$, where $R$ is the compacton radius (2.21).

Following the same procedure as adopted above, we calculate the pull-back of the metric $h$ induced by $\phi$ :

$$
\phi^{*} h=\frac{\partial \phi^{a}}{\partial x^{i}} \frac{\partial \phi^{a}}{\partial x^{j}} \mathrm{~d} x^{i} \otimes \mathrm{d} x^{j}=\frac{\partial \phi_{1}^{a}}{\partial x^{i}} \frac{\partial \phi_{1}^{a}}{\partial x^{j}} \mathrm{~d} x^{i} \otimes \mathrm{d} x^{j}+\frac{\partial \phi_{2}^{a}}{\partial x^{i}} \frac{\partial \phi_{2}^{a}}{\partial x^{j}} \mathrm{~d} x^{i} \otimes \mathrm{d} x^{j},
$$

where $\mathrm{d} x^{i}=(\mathrm{d} x, \mathrm{~d} y)$ are the Cartesian coordinates in $\mathcal{M}=\mathbb{R}^{2}$. In the last equality of eq. (3.28), we used the propriety of the compacton that the fields are constant outside of its radius so that

$$
\begin{aligned}
& \partial_{i} \phi^{a}(x, y)=\partial_{i} \phi_{1}^{a}\left(x+x_{0}, y\right)+\partial_{i} \phi_{2}^{a}\left(x-x_{0}, y\right) \\
& \partial_{i} \phi_{1}^{a}\left(x+x_{0}, y\right) \partial_{j} \phi_{2}^{a}\left(x-x_{0}, y\right)=0, \quad \forall \quad i, j .
\end{aligned}
$$

At this point it is useful to manipulate the expression (3.28) by a series of change of variables in order to write $\phi^{*} h$ as a sum of symmetric $(0,2)$ tensors of the form $(3.22)$. Hence,

$$
\begin{aligned}
\phi^{*} h & =\frac{\partial \phi_{1}^{a}}{\partial x^{i}} \frac{\partial \phi_{1}^{a}}{\partial x^{j}} \mathrm{~d} x^{i} \otimes \mathrm{d} x^{j}+\frac{\partial \phi_{2}^{a}}{\partial x^{i}} \frac{\partial \phi_{2}^{a}}{\partial x^{j}} \mathrm{~d} x^{i} \otimes \mathrm{d} x^{j} \\
& =\frac{\partial \phi_{1}^{a}}{\partial x_{+}^{i}} \frac{\partial \phi_{1}^{a}}{\partial x_{+}^{j}} \mathrm{~d} x_{+}^{i} \otimes \mathrm{d} x_{+}^{j}+\frac{\partial \phi_{2}^{a}}{\partial x_{-}^{i}} \frac{\partial \phi_{2}^{a}}{\partial x_{-}^{j}} \mathrm{~d} x_{-}^{i} \otimes \mathrm{d} x_{-}^{j} \\
& =\frac{\partial \phi_{1}^{a}}{\partial \tilde{x}_{+}^{i}} \frac{\partial \phi_{1}^{a}}{\partial \tilde{x}_{+}^{j}} \mathrm{~d} \tilde{x}_{+}^{i} \otimes \mathrm{d} \tilde{x}_{+}^{j}+\frac{\partial \phi_{2}^{a}}{\partial \tilde{x}_{-}^{i}} \frac{\partial \phi_{2}^{a}}{\partial \tilde{x}_{-}^{j}} \mathrm{~d} \tilde{x}_{-}^{i} \otimes \mathrm{d} \tilde{x}_{-}^{j},
\end{aligned}
$$

where $x_{ \pm}^{i}=\left(x_{ \pm}, y_{ \pm}\right)$are defined by

$$
x_{ \pm}=x \pm x_{0}, \quad y_{ \pm}=y,
$$


while $\tilde{x}_{ \pm}^{i}=\left(r_{ \pm}, \theta_{ \pm}\right)$are the polar coordinates

$$
r_{ \pm}=\sqrt{x_{ \pm}^{2}+y_{ \pm}^{2}}, \quad \theta_{ \pm}=\arctan \left(\frac{y_{ \pm}}{x_{ \pm}}\right) .
$$

Defining for simplicity

$$
\phi^{*} h \equiv a_{i j}^{+} \mathrm{d} \tilde{x}_{+}^{i} \otimes \mathrm{d} \tilde{x}_{+}^{j}+a_{i j}^{-} \mathrm{d} \tilde{x}_{-}^{i} \otimes \mathrm{d} \tilde{x}_{-}^{j},
$$

the final result for the two tensors is

$$
a_{i j}^{ \pm} \mathrm{d} \tilde{x}_{ \pm}^{i} \otimes \mathrm{d} \tilde{x}_{ \pm}^{j}=\left(f^{\prime}\left(r_{ \pm}\right)\right)^{2} \mathrm{~d} r_{ \pm}^{2}+N^{2} \sin ^{2} f\left(r_{ \pm}\right) \mathrm{d} \theta_{ \pm}^{2},
$$

which takes the same form as that of eq. (3.22), but with translated coordinates.

Applying the divergence to eq. (3.33) and using the linearity

$$
\begin{aligned}
\operatorname{div} \phi^{*} h=\operatorname{div}\left(a^{+}+a^{-}\right)=\operatorname{div} a^{+}+\operatorname{div} a^{-} & =\tilde{D}^{i} a_{i j}^{+} \mathrm{d} \tilde{x}_{+}^{j}+\tilde{D}^{i} a_{i j}^{-} \mathrm{d} \tilde{x}_{-}^{j} \\
& \equiv \omega_{j}^{+} \mathrm{d} \tilde{x}_{+}^{j}+\omega_{j}^{-} \mathrm{d} \tilde{x}_{-}^{j},
\end{aligned}
$$

with

$$
\omega_{j}^{ \pm} \mathrm{d} \tilde{x}_{ \pm}^{j}=\left(2 f^{\prime}\left(r_{ \pm}\right) f^{\prime \prime}\left(r_{ \pm}\right)+\frac{\left(f^{\prime}\left(r_{ \pm}\right)\right)^{2}}{r_{ \pm}}-N^{2} \frac{\sin ^{2} f\left(r_{ \pm}\right)}{r_{ \pm}^{3}}\right) \mathrm{d} r_{ \pm},
$$

we obtain a one-form whose exactness we must verify. Using Poincaré's lemma again as well as the linearity of the exterior derivative, we get

$$
\begin{aligned}
\mathrm{d}\left(\operatorname{div} \phi^{*} h\right) & =\mathrm{d}\left(\omega_{j}^{+} \mathrm{d} \tilde{x}_{+}^{j}+\omega_{j}^{-} \mathrm{d} \tilde{x}_{-}^{j}\right)=\sum_{ \pm} \mathrm{d}\left(\omega_{j}^{+} d \tilde{x}_{+}^{j}\right) \\
& =\sum_{ \pm}\left(\tilde{\partial}_{i} \omega_{j}^{ \pm}-\tilde{\partial}_{j} \omega_{i}^{ \pm}\right) \mathrm{d} \tilde{x}_{ \pm}^{i} \wedge \mathrm{d} \tilde{x}_{ \pm}^{j} \\
& =2 \sum_{ \pm}\left(\partial_{r_{ \pm}} \omega_{\theta}^{ \pm}-\partial_{\theta_{ \pm}} \omega_{r}^{ \pm}\right) \mathrm{d} r_{ \pm} \wedge \mathrm{d} \theta_{ \pm}=0,
\end{aligned}
$$

which finally proves that the map (3.25) is restricted harmonic.

The results of this section show that both an axially symmetric compacton with random orientation and two nonoverlapping axially-symmetric compactons, with random orientations are restricted harmonic maps. In this paper, we therefore use the first solution as the zeroth-order background for a single near-BPS baby Skyrmion, as already anticipated in section 3.1, and the second solution for two near-BPS baby Skyrmions.

With this last result, we are now able to calculate the energy of two near-BPS baby Skyrmions with topological charge $Q=N+N$ at the leading order in $\epsilon$, as already performed for the single case $Q=N$ in eq. (3.4). Using the restricted harmonic map (3.25) and the properties (3.29), we get

$$
\begin{aligned}
E^{\text {side-by-side }}(\epsilon, N \oplus N) & =M^{\mathrm{BPS}}(N \oplus N)+\epsilon M^{\mathrm{LO}}(N \oplus N) \\
& =2 M^{\mathrm{BPS}}(N)+2 \epsilon M^{\mathrm{LO}}(N),
\end{aligned}
$$

where $N \oplus N$ means an axially symmetric charge- $N$ compacton side-by-side of another axially symmetric charge- $N$ compacton. At this order, the energy of the $Q=N+N$ configuration is therefore simply the sum of the energies of the two components with no information about the interaction between two near-BPS baby Skyrmions. An investigation of the nextto-leading order (NLO) is required to calculate the binding energy of such a configuration. 


\subsection{NLO and $\mathrm{N}^{2} \mathrm{LO}$ corrections}

We will now consider the next-to-leading order (NLO) and next-to-next-to-leading order $\left(\mathrm{N}^{2} \mathrm{LO}\right)$ corrections to the energy, which corresponds to taking into account the corrections of order $\mathcal{O}\left(\epsilon^{2}\right)$ and $\mathcal{O}\left(\epsilon^{3}\right)$, respectively. To this end, we will perform a linear perturbation of the model

$$
\phi=\varphi+\delta \phi
$$

where $\delta \phi=\left(\delta \phi^{1}, \delta \phi^{2}, \delta \phi^{3}\right)$ is a small perturbation about the background solution $\boldsymbol{\varphi}=$ $\left(\varphi^{1}, \varphi^{2}, \varphi^{3}\right)$.

A brief spoiler of the results in this section, is that we need to go to the third order in $\epsilon$ to include the terms that will give rise to a nontrivial behavior of the fluctuations outside of the compacton domain(s). It will turn out to give, however, a nonanalytic contribution in $\epsilon$. Therefore, we will write down all the terms up to order $\mathcal{O}\left(\epsilon^{3}\right)$, but we will solve the equation of motion for the fluctuation to linearized order, which does indeed capture this nonanalytic contribution to the energy. A key step will be to find the correct form of the fluctuations that does not take the fields out of the $\mathrm{O}(3)$ group. For the NLO and $\mathrm{N}^{2} \mathrm{LO}$ corrections, we need to calculate the variation up to third order (in the fields) of the Lagrangian (2.1):

$$
\begin{aligned}
\mathcal{L}^{\text {perturb }}[\boldsymbol{\varphi}, \boldsymbol{\delta} \phi]= & \frac{\partial \mathcal{L}}{\partial \phi^{a}}\left|\delta \phi^{a}+\frac{1}{2} \frac{\partial^{2} \mathcal{L}}{\partial \phi^{a} \partial \phi^{b}}\right| \delta \phi^{a} \delta \phi^{b}+\frac{1}{6} \frac{\partial^{3} \mathcal{L}}{\partial \phi^{a} \partial \phi^{b} \partial \phi^{c}} \mid \delta \phi^{a} \delta \phi^{b} \delta \phi^{c} \\
& +\frac{\partial \mathcal{L}}{\partial \partial_{\mu} \phi^{a}}\left|\partial_{\mu} \delta \phi^{a}+\frac{1}{2} \frac{\partial^{2} \mathcal{L}}{\partial \partial_{\mu} \phi^{a} \partial \partial_{\nu} \phi^{b}}\right| \partial_{\mu} \delta \phi^{a} \partial_{\nu} \delta \phi^{b} \\
& +\frac{1}{6} \frac{\partial^{3} \mathcal{L}}{\partial \partial_{\mu} \phi^{a} \partial \partial_{\nu} \phi^{b} \partial \partial_{\rho} \phi^{c}} \mid \partial_{\mu} \delta \phi^{a} \partial_{\nu} \delta \phi^{b} \partial_{\rho} \delta \phi^{c} \\
= & \frac{\lambda_{0}+\delta \lambda}{2} \delta \phi^{2}+\delta \lambda \varphi \cdot \delta \phi+\epsilon \frac{\partial \mathcal{L}_{2}}{\partial \partial_{\mu} \phi^{a}} \mid \partial_{\mu} \delta \phi^{a} \\
& +\frac{1}{2} \frac{\partial^{2} \mathcal{L}}{\partial \partial_{\mu} \phi^{a} \partial \partial_{\nu} \phi^{b}}\left|\partial_{\mu} \delta \phi^{a} \partial_{\nu} \delta \phi^{b}+\frac{1}{6} \frac{\partial^{3} \mathcal{L}}{\partial \partial_{\mu} \phi^{a} \partial \partial_{\nu} \phi^{b} \partial \partial_{\rho} \phi^{c}}\right| \partial_{\mu} \delta \phi^{a} \partial_{\nu} \delta \phi^{b} \partial_{\rho} \delta \phi^{c} \\
= & \frac{\lambda_{0}+\delta \lambda}{2} \delta \phi^{2}+\delta \lambda \varphi \cdot \delta \phi-\epsilon J_{a}^{\mu} \partial_{\mu} \delta \phi^{a}-\frac{1}{2} V_{a b}^{\mu \nu} \partial_{\mu} \delta \phi^{a} \partial_{\nu} \delta \phi^{b} \\
& -\frac{1}{6} \Gamma_{a b c}^{\mu \nu \rho} \partial_{\mu} \delta \phi^{a} \partial_{\nu} \delta \phi^{b} \partial_{\rho} \delta \phi^{c},
\end{aligned}
$$

where the $\mid$ denotes that the expression (to the left of the bar) is evaluated on the background by setting $\phi=\varphi$ and we have defined

$$
J_{a}^{\mu} \equiv-\frac{\partial \mathcal{L}_{2}}{\partial \partial_{\mu} \phi^{a}}\left|=\partial^{\mu} \varphi^{a}, \quad V_{a b}^{\mu \nu} \equiv-\frac{\partial^{2} \mathcal{L}}{\partial \partial_{\mu} \phi^{a} \partial \partial_{\nu} \phi^{b}}\right|, \quad \Gamma_{a b c}^{\mu \nu \rho} \equiv-\frac{\partial^{3} \mathcal{L}}{\partial \partial_{\mu} \phi^{a} \partial \partial_{\nu} \phi^{b} \partial \partial_{\rho} \phi^{c}} \mid .
$$

We have furthermore replaced the Lagrange multiplier constant $\lambda$ with an expansion $\lambda \rightarrow \lambda_{0}+\delta \lambda$, where it is understood that $\lambda_{0}$ is the Lagrange multiplier that solves the sigma-model constraint for the background BPS solution. $\delta \lambda$ is then a Lagrange multiplier that ensures that the perturbation does not bring the total field $\phi=\varphi+\delta \phi$ out of the 
$\mathrm{O}(3)$ group, i.e. it should still preserve the unit length constraint $\phi \cdot \phi=1+\mathcal{O}\left(\epsilon^{4}\right)$. More precisely, if we vary the above Lagrangian density with respect to $\delta \lambda$ we obtain

$$
\left(\frac{1}{2} \boldsymbol{\delta} \phi^{2}+\varphi \cdot \delta \phi\right)=0
$$

which is solved by [12]

$$
\delta \phi=\Delta \times \varphi+\frac{1}{2} \Delta \times(\Delta \times \varphi),
$$

which satisfies the constraint (3.42) up to $\mathcal{O}\left(\boldsymbol{\Delta}^{4}\right)$, which we shall verify is of order $\mathcal{O}\left(\epsilon^{4}\right)$ and hence will give a contribution to the energy of order $\mathcal{O}\left(\epsilon^{5}\right)$. Notice that the above form automatically restricts $\boldsymbol{\Delta}$ to be orthogonal to the background solution $\boldsymbol{\varphi}$ to leading order.

The Hessian of the Lagrangian density with respect to the derivatives of the fields, $V_{a b}^{\mu \nu}$, is given by

$$
\begin{aligned}
V_{a b}^{\mu \nu} & \equiv V_{0 a b}^{\mu \nu}+\epsilon V_{1 a b}^{\mu \nu}, \\
V_{0 a b}^{\mu \nu} & \equiv\left(\partial_{\rho} \varphi \cdot \partial^{\rho} \varphi\right) \eta^{\mu \nu} \delta^{a b}+2 \partial^{\mu} \varphi^{a} \partial^{\nu} \varphi^{b}-\partial^{\mu} \varphi \cdot \partial^{\nu} \varphi \delta^{a b}-\partial_{\rho} \varphi^{a} \partial^{\rho} \varphi^{b} \eta^{\mu \nu}-\partial^{\mu} \varphi^{b} \partial^{\nu} \varphi^{a}, \\
V_{1 a b}^{\mu \nu} & \equiv \eta^{\mu \nu} \delta^{a b}
\end{aligned}
$$

the derivative of the Hessian is

$$
\begin{aligned}
\Gamma_{a b c}^{\mu \nu \rho}= & \eta^{\mu \nu}\left(2 \partial^{\rho} \varphi^{c} \delta^{a b}-\partial^{\rho} \varphi^{a} \delta^{b c}-\partial^{\rho} \varphi^{b} \delta^{c a}\right)+\eta^{\nu \rho}\left(2 \partial^{\mu} \varphi^{a} \delta^{b c}-\partial^{\mu} \varphi^{b} \delta^{c a}-\partial^{\mu} \varphi^{c} \delta^{a b}\right) \\
& +\eta^{\mu \rho}\left(2 \partial^{\nu} \varphi^{b} \delta^{c a}-\partial^{\nu} \varphi^{c} \delta^{a b}-\partial^{\nu} \varphi^{a} \delta^{b c}\right),
\end{aligned}
$$

and the Lagrange multiplier of the background, $\lambda_{0}$, reads

$$
\lambda_{0}=-\left(\boldsymbol{\varphi} \cdot \partial^{2} \boldsymbol{\varphi}\right)\left(\partial_{\mu} \boldsymbol{\varphi} \cdot \partial^{\mu} \boldsymbol{\varphi}\right)+\left(\boldsymbol{\varphi} \cdot \partial_{\mu} \partial_{\nu} \boldsymbol{\varphi}\right)\left(\partial^{\mu} \boldsymbol{\varphi} \cdot \partial^{\nu} \boldsymbol{\varphi}\right)-m^{2} \varphi^{3} .
$$

Substituting the form of the variation (3.43) into the perturbation Lagrangian density (3.40) yields

$$
\begin{aligned}
\mathcal{L}^{\text {perturb }}[\boldsymbol{\varphi}, \boldsymbol{\Delta}]= & \mathcal{L}_{2}^{\text {perturb }}[\boldsymbol{\varphi}, \boldsymbol{\Delta}]+\mathcal{L}_{3}^{\text {perturb }}[\boldsymbol{\varphi}, \boldsymbol{\Delta}]+\mathcal{O}\left(\epsilon^{4}\right) \\
\mathcal{L}_{2}^{\text {perturb }}[\boldsymbol{\varphi}, \boldsymbol{\Delta}]= & \frac{\lambda_{0}}{2}\left(\boldsymbol{\Delta}^{2}-(\boldsymbol{\Delta} \cdot \boldsymbol{\varphi})^{2}\right)-\epsilon \mathbf{J}^{\mu} \cdot \partial_{\mu} \boldsymbol{\Delta} \times \boldsymbol{\varphi}-\epsilon \mathbf{J}^{\mu} \cdot \boldsymbol{\Delta} \times \partial_{\mu} \boldsymbol{\varphi} \\
& -\frac{1}{2} V_{0 a b}^{\mu \nu} \varepsilon^{a c d} \partial_{\mu}\left(\Delta^{c} \varphi^{d}\right) \varepsilon^{b e f} \partial_{\nu}\left(\Delta^{e} \varphi^{f}\right) \\
\mathcal{L}_{3}^{\text {perturb }}[\boldsymbol{\varphi}, \boldsymbol{\Delta}]= & -\frac{\epsilon}{2}(\boldsymbol{\varphi} \cdot \boldsymbol{\Delta})\left(\mathbf{J}_{\mu} \cdot \partial^{\mu} \boldsymbol{\Delta}\right)-\frac{\epsilon}{2}\left(\mathbf{J}_{\mu} \cdot \boldsymbol{\Delta}\right)\left(\partial^{\mu} \boldsymbol{\varphi} \cdot \boldsymbol{\Delta}\right)-\frac{\epsilon}{2}\left(\mathbf{J}_{\mu} \cdot \boldsymbol{\Delta}\right)\left(\boldsymbol{\varphi} \cdot \partial^{\mu} \boldsymbol{\Delta}\right) \\
& +\frac{\epsilon}{2}\left(\mathbf{J}_{\mu} \cdot \partial^{\mu} \boldsymbol{\varphi}\right) \boldsymbol{\Delta}^{2}+\epsilon\left(\mathbf{J}_{\mu} \cdot \boldsymbol{\varphi}\right)\left(\boldsymbol{\Delta} \cdot \partial^{\mu} \boldsymbol{\Delta}\right) \\
& -\frac{1}{2} V_{0 a b}^{\mu \nu} \varepsilon^{a c d} \partial_{\mu}\left(\Delta^{c} \varphi^{d}\right) \partial_{\nu}\left(\Delta^{b}(\boldsymbol{\Delta} \cdot \boldsymbol{\varphi})-\boldsymbol{\Delta}^{2} \varphi^{b}\right) \\
& -\frac{1}{6} \Gamma_{a b c}^{\mu \nu \rho} \varepsilon^{a d e} \partial_{\mu}\left(\Delta^{d} \varphi^{e}\right) \varepsilon^{b f g} \partial_{\nu}\left(\Delta^{f} \varphi^{g}\right) \varepsilon^{c h i} \partial_{\rho}\left(\Delta^{h} \varphi^{i}\right) \\
& -\frac{\epsilon}{2} V_{1 a b}^{\mu \nu} \varepsilon^{a c d} \partial_{\mu}\left(\Delta^{c} \varphi^{d}\right) \varepsilon^{b e f} \partial_{\nu}\left(\Delta^{e} \varphi^{f}\right),
\end{aligned}
$$

which is the complete Lagrangian up to third order in $\epsilon$, (i.e. discarding terms of $\mathcal{O}\left(\epsilon^{4}\right)$ ). Notice that the term with $\delta \lambda$ is first needed at the fifth order in $\epsilon$ and can thus safely be ignored - its job was to produce the form (3.43). 
The reason for keeping terms up to third order in $\epsilon$, as we shall see, is to retain the last term in eq. (3.49), which will enable the fluctuation to propagate outside of the compacton and hence give rise to binding energy. In order to calculate the energy consistently, we have thus kept all other terms up to third order in $\epsilon$. However, for calculating $\boldsymbol{\Delta}$ we will linearize its equation of motion

$$
\begin{aligned}
& -\partial_{\mu}\left[V_{c b}^{\mu \nu} \varepsilon^{a c d} \varepsilon^{b e f} \partial_{\nu}\left(\Delta^{e} \varphi^{f}\right)\right] \varphi^{d}+\lambda_{0} \Delta^{a}-\lambda_{0}(\boldsymbol{\varphi} \cdot \boldsymbol{\Delta}) \varphi^{a}+\frac{\epsilon}{2}(\boldsymbol{\varphi} \cdot \boldsymbol{\Delta}) \partial_{\mu} J_{a}^{\mu}+\frac{\epsilon}{2}\left(\partial_{\mu} \mathbf{J}^{\mu} \cdot \boldsymbol{\Delta}\right) \varphi^{a} \\
& -\epsilon\left(\partial_{\mu} \mathbf{J}^{\mu} \cdot \boldsymbol{\varphi}\right) \Delta^{a}=-\epsilon \varepsilon^{a b c} \varphi^{b} \partial_{\mu} J_{c}^{\mu},
\end{aligned}
$$

where we have used that $V_{c b}^{\mu \nu}$ is symmetric under the simultaneous exchange of $\mu \leftrightarrow \nu$ and $c \leftrightarrow b$.

Let us now consider what happens outside of the compacton. Since $\varphi^{a} \rightarrow \delta^{a 3}$ is at its vacuum and all derivatives of the background field vanish identically, most terms including the source term (right-hand side of eq. (3.50)) switch off. In fact, the only remaining terms outside of the compacton background are $\epsilon V_{1 a b}^{\mu \nu} \subset V_{a b}^{\mu \nu}$ and the $\lambda_{0}$ terms in eq. (3.50). Thus the linearized equation of motion of the perturbation, $\Delta$, reduces outside of the compacton background to

$$
\epsilon\left(\partial^{2} \Delta^{a}-\partial^{2} \Delta^{3} \delta^{3 a}\right)-m^{2}\left(\Delta^{a}-\Delta^{3} \delta^{3 a}\right)=0 .
$$

It is interesting to see that the kinetic term here (outside the compacton) is given by $\epsilon V_{1 a b}^{\mu \nu}$ which is third order in $\epsilon$ (i.e. $\mathcal{O}\left(\epsilon^{3}\right)$ ). Thus, had we only kept terms up to second order in $\epsilon$ in the Lagrangian (3.40), the equation of motion would have been a constraint setting $\boldsymbol{\Delta}=0$. If $\boldsymbol{\Delta}=0$ outside the compacton, then there is no information propagating between two compactons and hence there is no binding energy.

A natural question then arises: at which order in $\epsilon$ is the binding energy of baby Skyrmions captured? If we do not restrict to the linearized equation of motion for the fluctuation, it is clear that the above conclusion about the equation outside of the compacton does not change (although we do not prove this). It is instructive to look at the solution for the fluctuation outside of the compacton as the solution to eq. (3.51), which reads

$$
\boldsymbol{\Delta}=\left(\begin{array}{c}
c_{1} \\
c_{2} \\
0
\end{array}\right) \exp \left(-\frac{m r}{\sqrt{\epsilon}}\right),
$$

where $c_{1,2}$ are constants. This solution illustrates perfectly the problem of describing the binding energy at a specific order. In fact, although the tail does not vanish for a nonvanishing $\epsilon$, any order in $\epsilon$ vanishes due to the exponential. In some sense, this solution is all-order in $\epsilon$ or "nonperturbative". Importantly, this shows that the expansion around the BPS compacton background contains contributions nonanalytic in $\epsilon$. A word of caution is that although we include this "nonperturbative" effect of the all-order tail for the linear perturbation, we solve a linearized equation for the perturbation which only captures every effect to second order in $\epsilon$ and some effects - like the crucial tail (3.52) at third order in $\epsilon$. Nevertheless, we calculate the full energy to third order in $\epsilon$ using this solution to the linearized equation of motion. 
Writing out eq. (3.50) explicitly in the static case, we get

$$
X \Delta_{i i}^{a}+X^{a b} \Delta_{i i}^{b}+X_{i j}^{a b} \Delta_{i j}^{b}+X_{i}^{a b} \Delta_{i}^{b}+\Lambda \Delta^{a}+\Lambda^{a b} \Delta^{b}=-\epsilon \varepsilon^{a b c} \varphi^{b} \varphi_{i i}^{c},
$$

where we have defined

$$
\begin{aligned}
X \equiv & \epsilon \\
X^{a b} \equiv & -\epsilon \varphi^{a} \varphi^{b}+\varphi_{j}^{a} \varphi_{j}^{b}, \\
X_{i j}^{a b} \equiv & -\varphi_{i}^{a} \varphi_{j}^{b}, \\
X_{i}^{a b} \equiv & -2 \epsilon \varphi_{i}^{a} \varphi^{b}-\varphi_{i j}^{a} \varphi_{j}^{b}+\varphi_{j j}^{a} \varphi_{i}^{b}-2 \varphi_{i}^{a} \varphi_{j j}^{b}+2 \varphi_{j}^{a} \varphi_{i j}^{b}+\left(\boldsymbol{\varphi}_{j} \cdot \boldsymbol{\varphi}_{j}\right)\left(\varphi^{a} \varphi_{i}^{b}-\varphi_{i}^{a} \varphi^{b}\right) \\
& -\left(\boldsymbol{\varphi}_{i} \cdot \boldsymbol{\varphi}_{j}\right)\left(\varphi^{a} \varphi_{j}^{b}-\varphi_{j}^{a} \varphi^{b}\right), \\
\Lambda \equiv & -m^{2} \varphi^{3}, \\
\Lambda^{a b} \equiv & -\frac{\epsilon}{2} \varphi_{i i}^{a} \varphi^{b}+\frac{\epsilon}{2} \varphi^{a} \varphi_{i i}^{b}+m^{2} \varphi^{3} \varphi^{a} \varphi^{b}-\left(\boldsymbol{\varphi}_{i j} \cdot \boldsymbol{\varphi}_{j}\right) \varphi_{i}^{a} \varphi^{b}+\left(\boldsymbol{\varphi}_{i i} \cdot \boldsymbol{\varphi}_{j}\right) \varphi_{j}^{a} \varphi^{b}+\left(\boldsymbol{\varphi}_{i} \cdot \boldsymbol{\varphi}_{j}\right) \varphi_{i j}^{a} \varphi^{b} \\
& -\left(\boldsymbol{\varphi}_{j} \cdot \boldsymbol{\varphi}_{j}\right) \varphi_{i i}^{a} \varphi^{b}+\left(\boldsymbol{\varphi}_{i} \cdot \boldsymbol{\varphi}_{j}\right)^{2} \varphi^{a} \varphi^{b}-\left(\boldsymbol{\varphi}_{i} \cdot \boldsymbol{\varphi}_{i}\right)^{2} \varphi^{a} \varphi^{b} .
\end{aligned}
$$

The energy density of the perturbation can be written as

$$
\mathcal{E}^{\text {perturb }}[\boldsymbol{\varphi}, \boldsymbol{\Delta}]=\mathcal{E}_{2}^{\text {perturb }}[\boldsymbol{\varphi}, \boldsymbol{\Delta}]+\mathcal{E}_{3}^{\text {perturb }}[\boldsymbol{\varphi}, \boldsymbol{\Delta}],
$$

with the second order (NLO) in $\epsilon$ :

$$
\begin{aligned}
\mathcal{E}_{2}^{\text {perturb }}[\boldsymbol{\varphi}, \boldsymbol{\Delta}]= & \epsilon \boldsymbol{\varphi}_{i} \cdot \boldsymbol{\Delta}_{i} \times \boldsymbol{\varphi}+\frac{m^{2}}{2} \boldsymbol{\varphi}^{3}\left(\boldsymbol{\Delta}^{2}-(\boldsymbol{\Delta} \cdot \boldsymbol{\varphi})^{2}\right) \\
& +\frac{1}{2}\left(\boldsymbol{\varphi}_{j} \cdot \boldsymbol{\varphi}_{j}\right)\left[\left(\boldsymbol{\varphi}_{i} \cdot \boldsymbol{\varphi}_{i}\right)(\boldsymbol{\varphi} \cdot \boldsymbol{\Delta})^{2}-\left(\boldsymbol{\varphi}_{i} \cdot \boldsymbol{\Delta}\right)^{2}-2(\boldsymbol{\varphi} \cdot \boldsymbol{\Delta})\left(\boldsymbol{\varphi}_{i} \cdot \boldsymbol{\Delta}_{i}\right)\right] \\
& -\frac{1}{2}\left(\boldsymbol{\varphi}_{i} \cdot \boldsymbol{\varphi}_{j}\right)\left[\left(\boldsymbol{\varphi}_{i} \cdot \boldsymbol{\varphi}_{j}\right)(\boldsymbol{\varphi} \cdot \boldsymbol{\Delta})^{2}-\left(\boldsymbol{\varphi}_{i} \cdot \boldsymbol{\Delta}\right)\left(\boldsymbol{\varphi}_{j} \cdot \boldsymbol{\Delta}\right)-2(\boldsymbol{\varphi} \cdot \boldsymbol{\Delta})\left(\boldsymbol{\varphi}_{i} \cdot \boldsymbol{\Delta}_{j}\right)\right] \\
& +\frac{1}{2}\left(\boldsymbol{\varphi}_{i} \cdot \boldsymbol{\Delta}_{i}\right)^{2}+\frac{1}{2}\left(\boldsymbol{\varphi}_{i} \cdot \boldsymbol{\Delta}_{j}\right)^{2}-\left(\boldsymbol{\varphi}_{i} \cdot \boldsymbol{\Delta}_{j}\right)\left(\boldsymbol{\varphi}_{j} \cdot \boldsymbol{\Delta}_{i}\right),
\end{aligned}
$$

the third order (NNLO) in $\epsilon$

$$
\begin{aligned}
\mathcal{E}_{3}^{\text {perturb }}[\boldsymbol{\varphi}, \boldsymbol{\Delta}]= & \mathcal{E}_{3, \text { quad }}^{\text {perturb }}[\boldsymbol{\varphi}, \boldsymbol{\Delta}]+\mathcal{E}_{3, \text { cubic }}^{\text {perturb }}[\boldsymbol{\varphi}, \boldsymbol{\Delta}] \\
\mathcal{E}_{3, \text { quad }}^{\text {perturb }}[\boldsymbol{\varphi}, \boldsymbol{\Delta}]= & \frac{\epsilon}{2} \boldsymbol{\Delta}_{i} \cdot \boldsymbol{\Delta}_{i}-\frac{\epsilon}{2}\left(\boldsymbol{\varphi} \cdot \boldsymbol{\Delta}_{i}\right)^{2}-\frac{\epsilon}{2}(\boldsymbol{\varphi} \cdot \boldsymbol{\Delta})\left(\boldsymbol{\varphi}_{i} \cdot \boldsymbol{\Delta}_{i}\right)+\frac{\epsilon}{2}\left(\boldsymbol{\varphi}_{i} \cdot \boldsymbol{\Delta}\right)\left(\boldsymbol{\varphi} \cdot \boldsymbol{\Delta}_{i}\right), \\
\mathcal{E}_{3, \text { cubic }}^{\text {perturb }}[\boldsymbol{\varphi}, \boldsymbol{\Delta}]= & -\frac{1}{3}\left(\boldsymbol{\varphi}_{i} \cdot \boldsymbol{\Delta}_{i}\right) \boldsymbol{\varphi}_{j} \cdot \boldsymbol{\Delta} \times \boldsymbol{\Delta}_{j}-\frac{1}{3}\left(\boldsymbol{\varphi}_{i} \cdot \boldsymbol{\Delta}_{j}\right) \boldsymbol{\varphi}_{i} \cdot \boldsymbol{\Delta} \times \boldsymbol{\Delta}_{j} \\
& +\frac{2}{3}\left(\boldsymbol{\varphi}_{i} \cdot \boldsymbol{\Delta}_{j}\right) \boldsymbol{\varphi}_{j} \cdot \boldsymbol{\Delta} \times \boldsymbol{\Delta}_{i}-\left(\boldsymbol{\varphi}_{i} \cdot \boldsymbol{\Delta}_{j}\right) \boldsymbol{\varphi} \cdot \boldsymbol{\Delta}_{i} \times \boldsymbol{\Delta}_{j} \\
& +\frac{1}{3}\left(\boldsymbol{\varphi}_{i} \cdot \boldsymbol{\varphi}_{i}\right)(\boldsymbol{\varphi} \cdot \boldsymbol{\Delta}) \boldsymbol{\varphi}_{j} \cdot \boldsymbol{\Delta} \times \boldsymbol{\Delta}_{j}-\frac{1}{3}\left(\boldsymbol{\varphi}_{i} \cdot \boldsymbol{\varphi}_{j}\right)(\boldsymbol{\varphi} \cdot \boldsymbol{\Delta}) \boldsymbol{\varphi}_{i} \cdot \boldsymbol{\Delta} \times \boldsymbol{\Delta}_{j} \\
& -\frac{1}{3}\left(\boldsymbol{\varphi}_{i} \cdot \boldsymbol{\Delta}\right)\left(\boldsymbol{\varphi}_{i} \cdot \boldsymbol{\Delta}\right) \boldsymbol{\varphi} \cdot \boldsymbol{\varphi}_{j} \times \boldsymbol{\Delta}_{j}+\frac{1}{3}\left(\boldsymbol{\varphi}_{i} \cdot \boldsymbol{\Delta}\right)\left(\boldsymbol{\varphi}_{j} \cdot \boldsymbol{\Delta}\right) \boldsymbol{\varphi} \cdot \boldsymbol{\varphi}_{i} \times \boldsymbol{\Delta}_{j} \\
& +\frac{5}{6}\left(\boldsymbol{\varphi}_{i} \cdot \boldsymbol{\varphi}_{i}\right)(\boldsymbol{\Delta} \cdot \boldsymbol{\Delta}) \boldsymbol{\varphi} \cdot \boldsymbol{\varphi}_{j} \times \boldsymbol{\Delta}_{j}-\frac{5}{6}\left(\boldsymbol{\varphi}_{i} \cdot \boldsymbol{\varphi}_{j}\right)(\boldsymbol{\Delta} \cdot \boldsymbol{\Delta}) \boldsymbol{\varphi} \cdot \boldsymbol{\varphi}_{i} \times \boldsymbol{\Delta}_{j} \\
& -\frac{1}{6}\left(\boldsymbol{\varphi}_{i} \cdot \boldsymbol{\varphi}_{i}\right)\left(\boldsymbol{\Delta} \cdot \boldsymbol{\Delta}_{j}\right) \boldsymbol{\varphi} \cdot \boldsymbol{\Delta} \times \boldsymbol{\varphi}_{j}+\frac{1}{6}\left(\boldsymbol{\varphi}_{i} \cdot \boldsymbol{\varphi}_{j}\right)(\boldsymbol{\Delta} \cdot \boldsymbol{\Delta}) \boldsymbol{\varphi} \cdot \boldsymbol{\Delta} \times \boldsymbol{\varphi}_{i}
\end{aligned}
$$




$$
\begin{aligned}
& +\frac{1}{2}\left(\boldsymbol{\varphi}_{i} \cdot \boldsymbol{\Delta}_{j}\right)(\boldsymbol{\varphi} \cdot \boldsymbol{\Delta}) \boldsymbol{\Delta} \cdot \boldsymbol{\varphi}_{i} \times \boldsymbol{\varphi}_{j}+\frac{1}{6}\left(\boldsymbol{\varphi}_{i} \cdot \boldsymbol{\Delta}_{j}\right)(\boldsymbol{\varphi} \cdot \boldsymbol{\Delta}) \boldsymbol{\varphi} \cdot \boldsymbol{\varphi}_{i} \times \boldsymbol{\Delta}_{j} \\
& -\frac{5}{6}\left(\boldsymbol{\varphi}_{i} \cdot \boldsymbol{\Delta}_{i}\right)(\boldsymbol{\varphi} \cdot \boldsymbol{\Delta}) \boldsymbol{\varphi} \cdot \boldsymbol{\varphi}_{j} \times \boldsymbol{\Delta}_{j}+\frac{2}{3}\left(\boldsymbol{\varphi}_{i} \cdot \boldsymbol{\Delta}_{j}\right)(\boldsymbol{\varphi} \cdot \boldsymbol{\Delta}) \boldsymbol{\varphi} \cdot \boldsymbol{\varphi}_{j} \times \boldsymbol{\Delta}_{i} \\
& +\frac{1}{6}\left(\boldsymbol{\varphi}_{i} \cdot \boldsymbol{\Delta}_{j}\right)\left(\boldsymbol{\varphi} \cdot \boldsymbol{\Delta}_{j}\right) \boldsymbol{\varphi} \cdot \boldsymbol{\Delta} \times \boldsymbol{\varphi}_{i}+\frac{1}{6}\left(\boldsymbol{\varphi}_{i} \cdot \boldsymbol{\Delta}_{i}\right)\left(\boldsymbol{\varphi} \cdot \boldsymbol{\Delta}_{j}\right) \boldsymbol{\varphi} \cdot \boldsymbol{\Delta} \times \boldsymbol{\varphi}_{j} \\
& -\frac{1}{3}\left(\boldsymbol{\varphi}_{i} \cdot \boldsymbol{\Delta}_{j}\right)\left(\boldsymbol{\varphi} \cdot \boldsymbol{\Delta}_{i}\right) \boldsymbol{\varphi} \cdot \boldsymbol{\Delta} \times \boldsymbol{\varphi}_{j}-\frac{1}{2}\left(\boldsymbol{\varphi}_{i} \cdot \boldsymbol{\varphi}_{i}\right)\left(\boldsymbol{\varphi}_{j} \cdot \boldsymbol{\Delta}\right) \boldsymbol{\varphi} \cdot \boldsymbol{\Delta} \times \boldsymbol{\Delta}_{j} \\
& +\frac{1}{2}\left(\boldsymbol{\varphi}_{i} \cdot \boldsymbol{\varphi}_{j}\right)\left(\boldsymbol{\varphi}_{i} \cdot \boldsymbol{\Delta}\right) \boldsymbol{\varphi} \cdot \boldsymbol{\Delta} \times \boldsymbol{\Delta}_{j}+\frac{1}{6}\left(\boldsymbol{\varphi}_{i} \cdot \boldsymbol{\Delta}\right)\left(\boldsymbol{\varphi}_{i} \cdot \boldsymbol{\Delta}_{j}\right) \boldsymbol{\varphi} \cdot \boldsymbol{\Delta} \times \boldsymbol{\varphi}_{j} \\
& +\frac{1}{6}\left(\boldsymbol{\varphi}_{i} \cdot \boldsymbol{\Delta}\right)\left(\boldsymbol{\varphi}_{j} \cdot \boldsymbol{\Delta}_{j}\right) \boldsymbol{\varphi} \cdot \boldsymbol{\Delta} \times \boldsymbol{\varphi}_{i}-\frac{1}{3}\left(\boldsymbol{\varphi}_{i} \cdot \boldsymbol{\Delta}\right)\left(\boldsymbol{\varphi}_{j} \cdot \boldsymbol{\Delta}_{i}\right) \boldsymbol{\varphi} \cdot \boldsymbol{\Delta} \times \boldsymbol{\varphi}_{j}
\end{aligned}
$$

where the former expression contains only quadratic terms in $\boldsymbol{\Delta}$ which contribute to the linear equation of motion and the latter gathers the cubic terms.

Notice that the entire perturbation energy vanishes manifestly for $\Delta \propto \boldsymbol{\varphi}$. Due to the nonlinearity in the form of $\boldsymbol{\delta} \phi$ in eq. (3.43), a mixing can occur if both a transverse $\boldsymbol{\Delta}_{\perp}$ and a longitudinal perturbation $\delta c(x) \boldsymbol{\varphi}$ is turned on at the same time. Inserting $\boldsymbol{\Delta}=\delta c \boldsymbol{\varphi}+\boldsymbol{\Delta}_{\perp}$ into quadratic part of the perturbation Lagrangian yields

$$
\mathcal{L}_{2}^{\text {perturb }}+\mathcal{L}_{3, \text { quad }}^{\text {perturb }} \supset \frac{\epsilon}{2}\left[\delta c \partial_{\mu} \boldsymbol{\varphi} \cdot \partial^{\mu} \boldsymbol{\Delta}_{\perp}+\partial_{\mu} \delta c \partial^{\mu} \boldsymbol{\varphi} \cdot \boldsymbol{\Delta}_{\perp}\right]
$$

The solution for $\delta c$ - which is sourceless - with vanishing boundary condition at $r=0$ and $r \rightarrow \infty$ (as measured from the compacton origin) yields $\delta c=0$. Because of the absence of a quadratic term for $\delta c$, variation neither gives an auxiliary equation (algebraic) nor a dynamic equation for $\delta c$. We checked that the quadratic terms in $\delta c$ do not appear in the cubic part of the Lagrangian either and hence can first appear at order $\mathcal{O}\left(\epsilon^{4}\right)$, which we shall not consider in this paper. ${ }^{3}$ We shall thus only consider $\boldsymbol{\Delta}=\boldsymbol{\Delta}_{\perp}$ which is transverse to the background solution $\boldsymbol{\varphi}$, i.e. for which it holds $\boldsymbol{\varphi} \cdot \boldsymbol{\Delta}_{\perp}=0$.

At this point, it will prove useful to specialize to the case of the background BPS solution for $\boldsymbol{\varphi}$, the transverse perturbations for $\boldsymbol{\Delta}=\boldsymbol{\Delta}_{\perp}$ and switch to polar coordinates in $\mathbb{R}^{2}$, for which the perturbation energy reads

$$
\mathcal{E}^{\text {perturb }}[f, \delta f, \delta \theta]=\mathcal{E}_{2}^{\text {perturb }}[f, \delta f, \delta \theta]+\mathcal{E}_{3, \text { quad }}^{\text {perturb }}[f, \delta f, \delta \theta]+\mathcal{E}_{3, \text { cubic }}^{\text {perturb }}[f, \delta f, \delta \theta],
$$

with

$$
\begin{aligned}
\mathcal{E}_{2}^{\text {perturb }}[f, \delta f, \delta \theta]=\frac{\epsilon N^{2}}{2 r^{2}} \sin (2 f) \delta f+\epsilon f_{r} \delta f_{r}+\frac{N^{2}}{2 r^{2}} \sin ^{2}(f) \delta f_{r}^{2}+\frac{f_{r}^{2}}{2 r^{2}} \delta \theta_{\theta}^{2} \\
+\frac{1}{2}\left(m^{2} \cos f+\frac{N^{2}}{r^{2}} \cos (2 f) f_{r}^{2}\right) \delta f^{2}+\frac{1}{2}\left(m^{2} \cos f-\frac{N^{2}}{r^{2}} \sin ^{2}(f) f_{r}^{2}\right) \delta \theta^{2} \\
+\frac{N}{r^{2}} \sin (f) f_{r}\left[2 \delta f_{r} \delta \theta_{\theta}-\delta f_{\theta} \delta \theta_{r}\right]+\frac{N^{2}}{2 r^{2}} \sin (2 f) f_{r}\left[2 \delta f_{r} \delta f+\delta \theta_{r} \delta \theta\right]+\frac{N}{r^{2}} \cos (f) f_{r}^{2} \delta f \delta \theta_{\theta}
\end{aligned}
$$

\footnotetext{
${ }^{3}$ We are working with the perturbation of the form (3.43) for which $\phi \cdot \phi=1+\mathcal{O}\left(\epsilon^{4}\right)$, so this relation should be improved to higher order before making conclusions about the terms present at order $\mathcal{O}\left(\epsilon^{4}\right)$. Then we expect in such case the quadratic terms of $\delta c$ would not even appear at order $\mathcal{O}\left(\epsilon^{4}\right)$, but that is far beyond the scope of this paper.
} 
for the NLO terms,

$$
\begin{aligned}
\mathcal{E}_{3, \text { quad }}^{\text {perturb }}[f, \delta f, \delta \theta]= & \frac{\epsilon}{2}\left(\delta f_{r}^{2}+\frac{\delta f_{\theta}^{2}}{r^{2}}\right)+\frac{\epsilon N^{2}}{2 r^{2}} \cos (2 f) \delta f^{2}+\frac{\epsilon}{2}\left(\delta \theta_{r}^{2}+\frac{\delta \theta_{\theta}^{2}}{r^{2}}\right) \\
& -\frac{\epsilon}{2}\left(f_{r}^{2}-\frac{N^{2}}{r^{2}} \cos ^{2} f\right) \delta \theta^{2}-\frac{\epsilon N}{r^{2}} \cos (f)\left(\delta f_{\theta} \delta \theta-\delta f \delta \theta_{\theta}\right),
\end{aligned}
$$

for the NNLO terms quadratic in $\boldsymbol{\Delta}$ and

$$
\begin{aligned}
& \mathcal{E}_{3, \text { cubic }}^{\text {perturb }}[f, \delta f, \delta \theta]=\frac{1}{2 r^{2}}\left(N \sin (f) \delta f_{r}+f_{r} \delta \theta_{\theta}+N \cos (f) f_{r} \delta f\right) \\
& \times\left[2 N \cos (f) \delta f_{r} \delta f+2 \delta f_{r} \delta \theta_{\theta}+2 N \cos (f) \delta \theta_{r} \delta \theta-2 \delta f_{\theta} \delta \theta_{r}-N \sin (f) f_{r} \delta f^{2}-N \sin (f) f_{r} \delta \theta^{2}\right]
\end{aligned}
$$

for the NNLO terms cubic in $\boldsymbol{\Delta}$, and we have defined the transverse $\boldsymbol{\Delta}$ perturbations

$$
\boldsymbol{\Delta}_{\perp} \equiv\left(\begin{array}{c}
-\sin N \theta \\
\cos N \theta \\
0
\end{array}\right) \delta f-\left(\begin{array}{c}
\cos f \cos N \theta \\
\cos f \sin N \theta \\
-\sin f
\end{array}\right) \delta \theta
$$

and the background BPS compacton solution is described by the radial profile

$$
f=f^{\mathrm{BPS}}=\arccos \left(-1+\frac{4 r^{2}}{R^{2}}-\frac{2 r^{4}}{R^{4}}\right),
$$

with $R$ being the compacton radius of eq. (2.21).

The corresponding equations of motion can be written as

$$
\begin{aligned}
X_{r r}\left(\begin{array}{c}
\delta f_{r r} \\
\delta \theta_{r r}
\end{array}\right)+\frac{1}{r} X_{r}\left(\begin{array}{c}
\delta f_{r} \\
\delta \theta_{r}
\end{array}\right)+ & \frac{1}{r^{2}} X_{\theta \theta}\left(\begin{array}{c}
\delta f_{\theta \theta} \\
\delta \theta_{\theta \theta}
\end{array}\right)+\frac{1}{r} X_{\theta}\left(\begin{array}{c}
\delta f_{\theta} \\
\delta \theta_{\theta}
\end{array}\right)+\frac{1}{r} X_{r \theta}\left(\begin{array}{c}
\delta f_{r \theta} \\
\delta \theta_{r \theta}
\end{array}\right) \\
+\Lambda\left(\begin{array}{c}
\delta f \\
\delta \theta
\end{array}\right) & =\left(\begin{array}{c}
-\epsilon\left(f_{r r}+\frac{1}{r} f_{r}-\frac{N^{2}}{2 r^{2}} \sin (2 f)\right) \\
0
\end{array}\right),
\end{aligned}
$$


where we have defined the matrices

$$
\begin{aligned}
X_{r r} & =\left(\begin{array}{cc}
\epsilon+\frac{N^{2}}{r^{2}} \sin ^{2} f & 0 \\
0 & \epsilon
\end{array}\right), \\
X_{r} & =\left(\begin{array}{cc}
\epsilon-\frac{N^{2}}{r^{2}} \sin ^{2} f+\frac{N^{2}}{r} \sin (2 f) f_{r} & 0 \\
0 & \epsilon
\end{array}\right), \\
X_{\theta \theta} & =\left(\begin{array}{cc}
\epsilon & 0 \\
0 & \epsilon+f_{r}^{2}
\end{array}\right) \\
X_{\theta} & =\left(\begin{array}{cc}
0 & X_{\theta}^{\delta \theta} \\
X_{\theta}^{\delta f} & 0
\end{array}\right), \\
X_{\theta}^{\delta f} & =\frac{2 \epsilon N}{r} \cos f-\frac{N}{r} \sin (f) f_{r r}+\frac{N}{r^{2}} \sin (f) f_{r}, \\
X_{\theta}^{\delta \theta} & =-\frac{2 \epsilon N}{r} \cos f+\frac{2 N}{r} \sin (f) f_{r r}+\frac{N}{r} \cos (f) f_{r}^{2}-\frac{2 N}{r^{2}} \sin (f) f_{r}, \\
X_{r \theta} & =\frac{N}{r} \sin (f) f_{r}\left(\begin{array}{ll}
0 & 1 \\
1 & 0
\end{array}\right), \\
\Lambda & =\left(\begin{array}{cc}
\Lambda^{\delta f} & 0 \\
0 & \Lambda^{\delta \theta}
\end{array}\right), \\
\Lambda^{\delta f} & =-\frac{\epsilon N^{2}}{r^{2}} \cos (2 f)+\frac{N^{2}}{r^{2}} \sin (2 f) f_{r r}+\frac{N^{2}}{r^{2}} \cos (2 f) f_{r}^{2}-\frac{N^{2}}{r^{3}} \sin (2 f) f_{r}-m^{2} \cos f, \\
\Lambda^{\delta \theta} & =\epsilon f_{r}^{2}-\frac{\epsilon N^{2}}{r^{2}} \cos ^{2}(f)+\frac{N^{2}}{2 r^{2}} \sin (2 f) f_{r r}+\frac{N^{2}}{r^{2}} \cos ^{2}(f) f_{r}^{2}-\frac{N^{2}}{2 r^{3}} \sin (2 f) f_{r}-m^{2} \cos f .
\end{aligned}
$$

First notice that there is only a source term (right-hand side of eq. (3.72)) for the upper equation, viz. for $\delta f$. Notice then that the off-diagonal components reside only in the matrices $X_{\theta}, X_{r \theta}$ and hence couple the second equation to $\delta f_{\theta}$ and $\delta f_{r \theta}$. The source for the $\delta \theta$ perturbation is thus the coupling to the nontrivial $\theta$ behavior in $\delta f$. It is now clear that, if we restrict to axially symmetric perturbations such that $\delta f=\delta f(r)$, the second equation decouples and is sourceless; hence it is satisfied by the trivial solution $\delta \theta=0$, everywhere.

The perturbation governing axially symmetric baby Skyrmions is thus simply described by the equation

$$
\begin{aligned}
\left(\epsilon+\frac{N^{2}}{r^{2}} \sin ^{2} f\right) \delta f_{r r}+\frac{1}{r}\left(\epsilon-\frac{N^{2}}{r^{2}} \sin ^{2} f\right. & \left.+\frac{N^{2}}{r} \sin (2 f) f_{r}\right) \delta f_{r}+\Lambda^{\delta f} \delta f \\
& =-\epsilon\left(f_{r r}+\frac{1}{r} f_{r}-\frac{N^{2}}{2 r^{2}} \sin 2 f\right)
\end{aligned}
$$

and the corresponding energy for the axially symmetric perturbation is

$$
\begin{aligned}
\mathcal{E}_{2}^{\text {perturb }}[f, \delta f]= & \frac{\epsilon N^{2}}{2 r^{2}} \sin (2 f) \delta f+\epsilon f_{r} \delta f_{r}+\frac{N^{2}}{2 r^{2}} \sin ^{2}(f) \delta f_{r}^{2} \\
& +\frac{1}{2}\left(m^{2} \cos f+\frac{N^{2}}{r^{2}} \cos (2 f) f_{r}^{2}\right) \delta f^{2}+\frac{N^{2}}{r^{2}} \sin (2 f) f_{r} \delta f_{r} \delta f,
\end{aligned}
$$


for the NLO terms,

$$
\mathcal{E}_{3, \text { quad }}^{\text {perturb }}[f, \delta f]=\frac{\epsilon}{2} \delta f_{r}^{2}+\frac{\epsilon N^{2}}{2 r^{2}} \cos (2 f) \delta f^{2},
$$

for the NNLO terms quadratic in $\delta f$ and

$$
\mathcal{E}_{3, \text { cubic }}^{\text {perturb }}[f, \delta f]=\frac{N^{2}}{2 r^{2}} \sin (2 f) \delta f_{r}^{2} \delta f+\frac{N^{2}}{r^{2}}\left(1-\frac{3}{2} \sin ^{2} f\right) f_{r} \delta f_{r} \delta f^{2}-\frac{N^{2}}{4 r^{2}} \sin (2 f) f_{r}^{2} \delta f^{3},
$$

for the NNLO terms cubic in $\delta f$.

\subsection{Axially symmetric solutions}

In order to verify our perturbative scheme, we start with axially symmetric baby Skyrmions. It will prove useful to interpret the perturbation $\delta f$ as follows

$$
\begin{aligned}
\phi & =\boldsymbol{\varphi}+\boldsymbol{\delta} \phi \\
& =\boldsymbol{\varphi}+\boldsymbol{\Delta}_{\perp} \times \boldsymbol{\varphi}+\frac{1}{2} \boldsymbol{\Delta}_{\perp} \times\left(\boldsymbol{\Delta}_{\perp} \times \boldsymbol{\varphi}\right) \\
& =\left(\begin{array}{c}
\sin f \cos N \theta \\
\sin f \sin N \theta \\
\cos f
\end{array}\right)+\left(\begin{array}{c}
\cos f \cos N \theta \\
\cos f \sin N \theta \\
-\sin f
\end{array}\right) \delta f+\frac{1}{2}\left(\begin{array}{c}
-\sin f \cos N \theta \\
-\sin f \sin N \theta \\
-\cos f
\end{array}\right) \delta f^{2} \\
& \simeq\left(\begin{array}{c}
\sin (f+\delta f) \cos N \theta \\
\sin (f+\delta f) \sin N \theta \\
\cos (f+\delta f)
\end{array}\right)+\mathcal{O}\left(\delta f^{3}\right)
\end{aligned}
$$

where we have used eq. (3.70) and set $\delta \theta=0$. It is now clear that $\delta f$ indeed is an additive correction to the BPS background profile function $f$.

The naive attempt is to solve eq. (3.83) with boundary conditions

$$
\delta f(0)=0, \quad \delta f(\infty)=0 .
$$

Had the soliton background solution been smooth, it would probably have worked out. However, the first derivative of the background solution is discontinuous at $r=R$ with the compacton radius $R$ given by eq. (2.21). Since the full soliton solution is smooth, the perturbation should counteract this cusp (jump in the first derivative), which we can write as a new condition at $r=R$ :

$$
\lim _{r \rightarrow R^{-}} \partial_{r} \phi^{3}-\lim _{r \rightarrow R^{+}} \partial_{r} \phi^{3}=0,
$$

which with the perturbation $\delta f$ of eq. (3.70) yields

$$
\partial_{r} \delta f\left(R^{-}\right)-\partial_{r} \delta f\left(R^{+}\right)=\frac{4}{R} .
$$

We will refer to this extra condition in addition to the conditions (3.88) as the "new boundary conditions." 

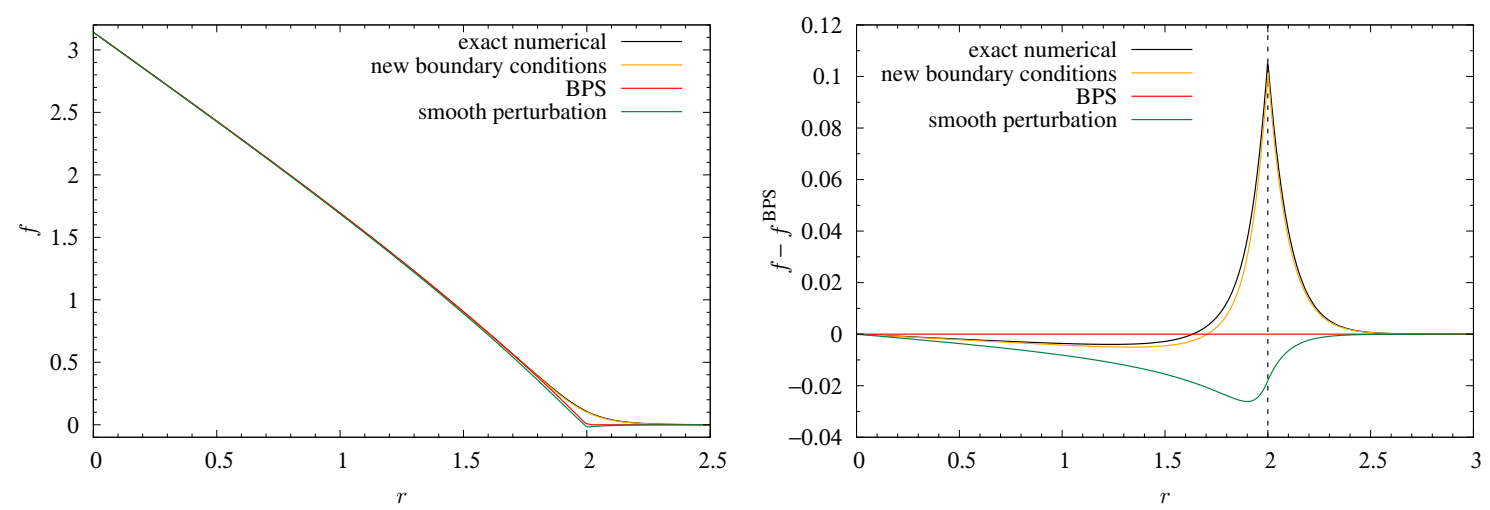

Figure 1. The profile function $f=\arccos \left(\phi^{3}\right)$ for $N=1$ and $\epsilon=0.01$ with $m=1$. The BPS background profile function is shown as a red solid curve. The smooth perturbation (dark-green curve) does not capture the kink at the compacton radius $r=R=2$. The perturbation subject to the new boundary condition (3.90) (orange) on the other hand lies very close to the exact numerical solution (black).

In figure 1 is shown the profile function $f=\arccos \left(\phi^{3}\right)$ for the $N=1$ axially symmetric baby Skyrmion with $\epsilon=0.01$ obtained by different methods. Since the BPS solution is a good starting point, we subtract off the BPS profile to better see the differences in the panel on the right-hand side. The naive implementation with a smooth perturbation is shown as the dark-green curve and does not resemble an improvement with respect to the BPS background solution compared to the exact solution (numerical). The perturbation subject to the new boundary condition (3.90) (orange curve) on the other hand gives an incredible improvement over the BPS solution and the discrepancy with respect to the exact solution (numerical) is really tiny and is expected to be due to nonlinearities (since we have linearized its equation of motion (3.83)).

We are now ready to calculate the perturbative corrections to the energy of the $N=1,2,4$ axially symmetric baby Skyrmions and the results are shown in figure 2 . The corrections are calculated using the linear perturbation, which is a solution to equation (3.83) subject to the new boundary condition (3.90). The NLO energy is calculated using the energy to order $\epsilon^{2}$ using eq. (3.84), whereas the $\mathrm{N}^{2} \mathrm{LO}$ energy is calculated as the sum of eqs. (3.85) and (3.86). We can see that the perturbative LO corrections overshoot the energy for every $N$ and the NLO corrections correct this overshooting too strongly, yielding an undershoot. Including finally the $\mathrm{N}^{2} \mathrm{LO}$ corrections, the result is extremely close to the exact numerical one. Notice by careful inspection of the figure, that the red crosses actually match the exact result (black curves) better than the fit (dark-blue curves).

The fit to the $\mathrm{N}^{2} \mathrm{LO}$ data (for $m=1$ ) gives

$$
\begin{aligned}
E(\epsilon, N)= & M^{\mathrm{BPS}}(N)+\epsilon M^{\mathrm{LO}}(N)+\epsilon^{2} M^{\mathrm{NLO}}(N)+\epsilon^{3} M^{\mathrm{N}^{2} \mathrm{LO}}(N)+\epsilon^{4} M^{\text {residual }, 4}(N) \\
= & \frac{16 \pi N}{3}+\epsilon\left(8 \pi \log 2+\frac{7 \pi N^{2}}{6}\right)+\epsilon^{2}\left(-128.9+43.50 N-6.869 N^{2}\right) \\
& +\epsilon^{3}\left(1458-450.9 N+57.20 N^{2}\right)+\epsilon^{4}\left(-6044+1884 N-238.4 N^{2}\right) .
\end{aligned}
$$



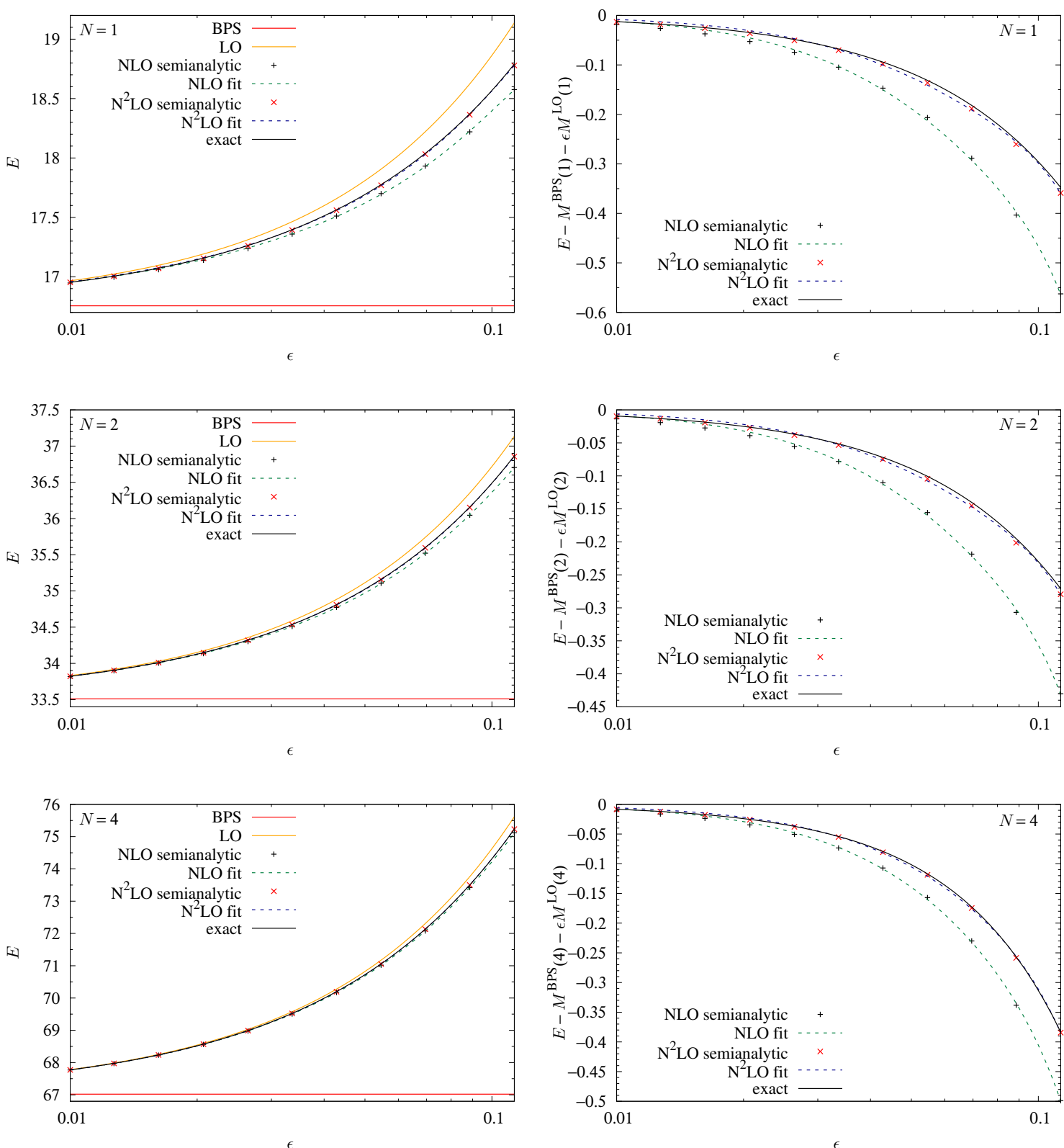

Figure 2. The mass of the baby Skyrmions in the perturbative scheme as a function of $\epsilon$ (on a logarithmic scale): the red line is the BPS bound, the orange curve is the leading order (LO) correction, the black pluses are the corrections calculated at next-to-leading order (NLO) using the linear perturbation, the green-dashed curve is a fit to the latter points, the red crosses are the corrections calculated at next-to-next-to-leading order $\left(\mathrm{N}^{2} \mathrm{LO}\right)$ using the same linear perturbation, the dark-blue curve is a fit to the latter points and the black curve is the exact energy calculated using the full (nonlinear) equations of motion. The left columns show the total energy while the right columns show the energy relative to the LO correction. 


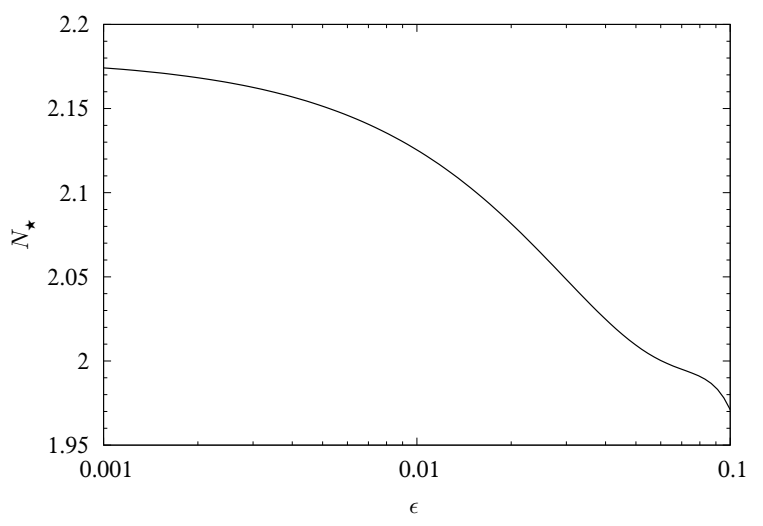

Figure 3. The critical value, $N_{\star}$, of the topological charge of the axially symmetric baby Skyrmion, as a function of $\epsilon . N_{\star}$ corresponds to the smallest energy per topological charge and hence the globally stable axially symmetric configuration.

This higher-order result in turn gives a correction to $N_{\star}$ of eq. (3.6):

$$
N_{\star}=2.180-6.023 \epsilon+54.60 \epsilon^{2}+55.52 \epsilon^{3}-2083 \epsilon^{4}+\mathcal{O}\left(\epsilon^{5}\right) .
$$

Although we write the expansion to fourth order in $\epsilon$, it should not be trusted beyond the third order. The higher orders simply represent the all-order contributions from the tail that are important for capturing the binding energies. $N_{\star}$ is shown in figure 3 . As can be seen from the figure, taking into account higher-order corrections in $\epsilon$ decreases $N_{\star}$. However, the $N=2$ baby Skyrmion remains the energetically stable axially symmetric solution. The drop in $N_{\star}$ after $\epsilon \gtrsim 0.1$ is possibly just an artifact of the fit and should not be taken too seriously at this stage.

\section{Numerical calculations}

In this section, we present numerical solutions to the full equations of motion. This is a computationally extremely expensive work and can be carried out only for the 2-dimensional case unless very clever adaptive methods are being used. The numerical calculations performed here are carried out with a fourth-order 5-point stencil finite-difference method using the arrested Newton flow [35] on a square grid of sizes up to $\sim 3226^{2} \sim 10^{7}$ with lattice spacing down to $\sim 0.0037$. Our numerical accuracy is about $10^{-6}$ or better. Such expensive grids would take a very long time on conventional CPU clusters, so our code is written in CUDA C and is executed on a GPU cluster. In order to avoid warp divergence, the code is made such that the bulk of the lattice is launched as one kernel without any conditionals and the edges are launched as two different additional kernels corresponding to the vertical and horizontal edges of the lattice (also without any conditionals).

In this section we explore the numerical solutions of baby Skyrmions that do not possess axial symmetry, however, we also check the axially symmetric configurations both for ensuring that they exist in the given range of parameter space and for comparing our numerical accuracy to that obtained using ODEs for the axially symmetric system. 

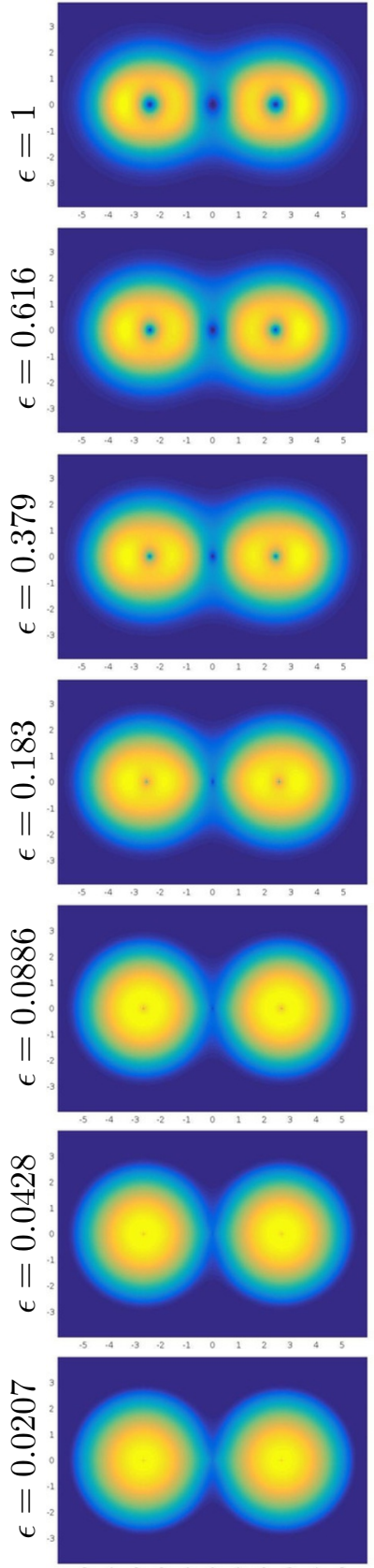
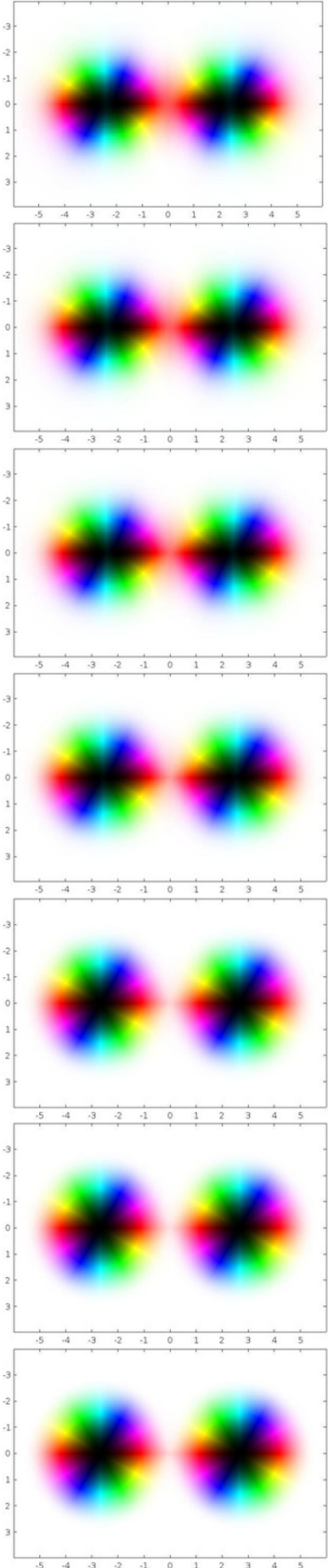
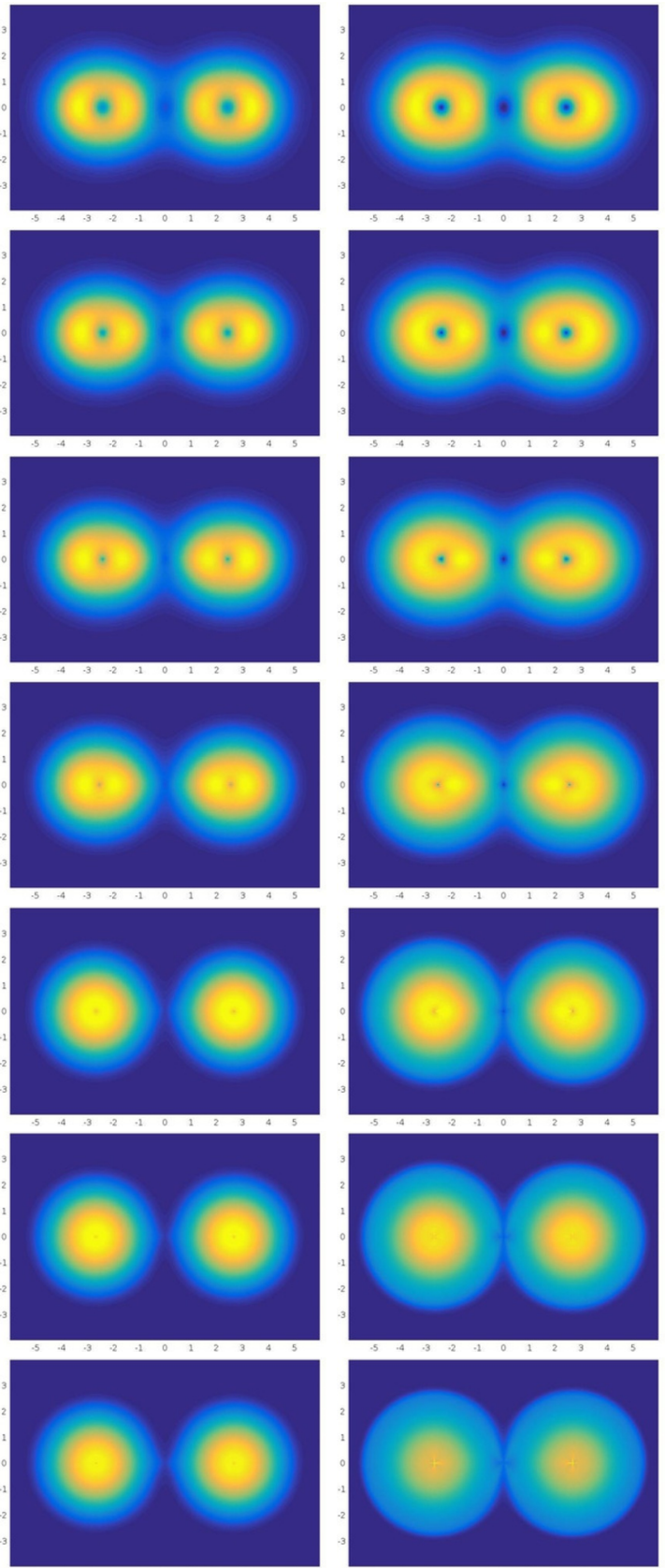

Figure 4. The $Q=2+2$ baby Skyrmion solution as a function of $\epsilon$, which is the stable $Q=4$ solution. The columns display the topological charge density, the field orientation using the color scheme described in the text, the total energy and finally the kinetic term $-\epsilon \mathcal{L}_{2}$. We note that the two stretched $N=2$ baby Skyrmions become almost perfectly round in the last row (for $\epsilon=0.0207$ ).

Our aim here is to confirm whether the energetically most favorable configuration in the case of small but finite $0<\epsilon \ll 1$ - consists of $N=2$ baby Skyrmions sitting next to each other at a finite or vanishing distance between them. Such a scenario is supported by the notion of restricted harmonic maps. 

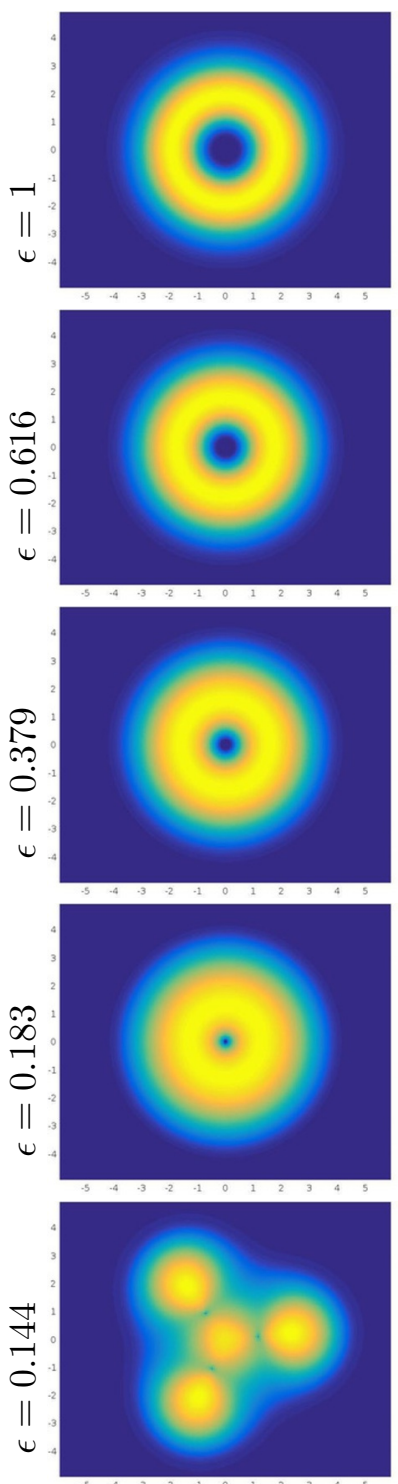
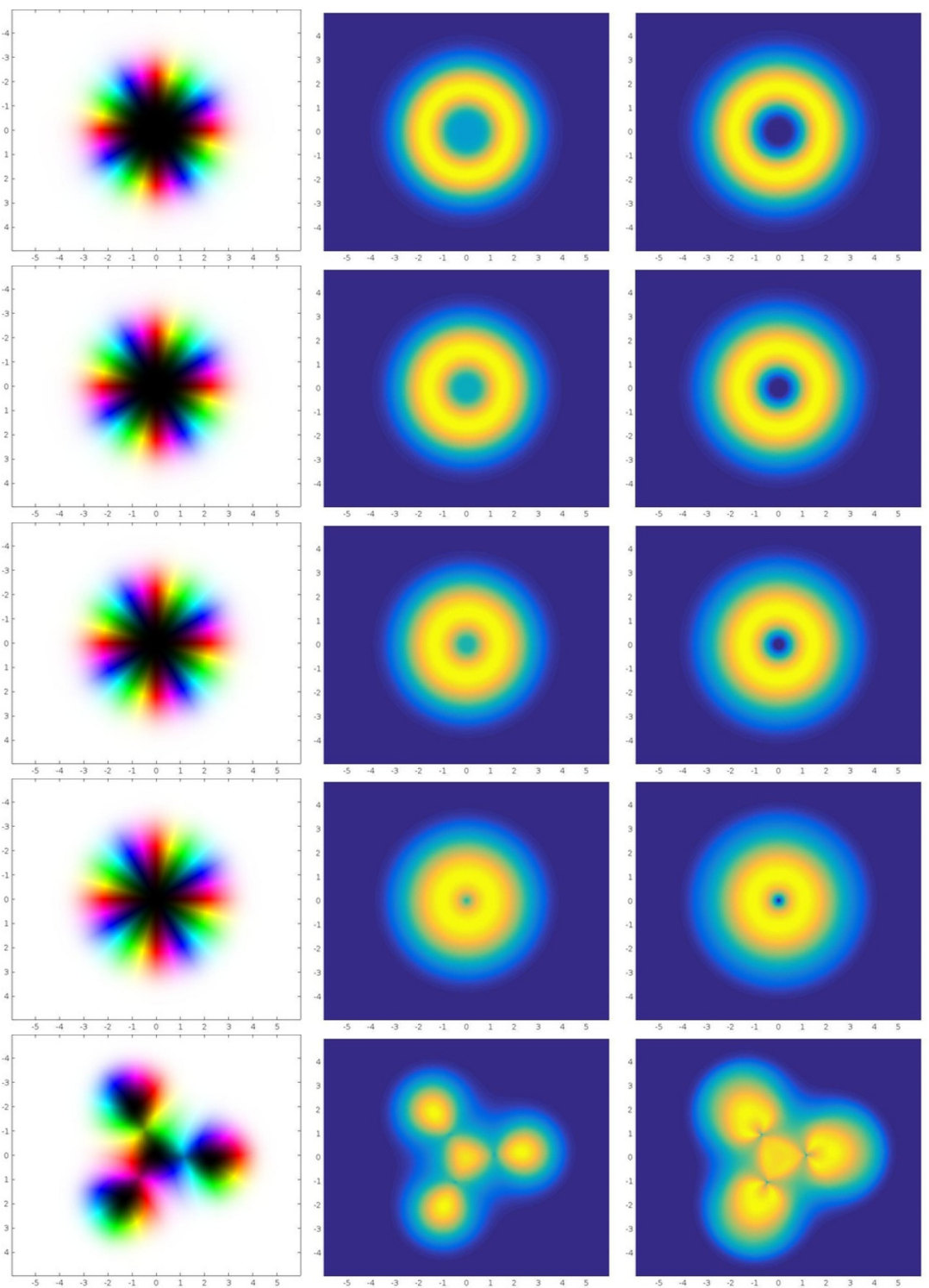

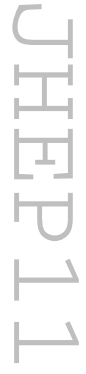

Figure 5. The $N=4$ baby Skyrmion solution as a function of $\epsilon$. The columns display the topological charge density, the field orientation using the color scheme described in the text, the total energy and finally the kinetic term $-\epsilon \mathcal{L}_{2}$. The last row (i.e. for $\epsilon=0.144$ ) shows that the solution has decayed into a lower-energy state with tetrahedral symmetry instead of axial symmetry.

To this end, we start with two $N=2$ baby Skyrmions (hence with a total topological charge $Q=2+2=4$ ), situated next to each other. Some of the numerical results are shown in figure 4 . The figure is organized into four columns showing the topological charge density, the field configuration using a color scheme, the total energy density and finally the energy density of the kinetic term $\left(-\epsilon \mathcal{L}_{2}\right)$. The colors on each graph is relatively rescaled to the values of the graph. The color scheme for the second column of figure 4 illustrates the orientation of the fields at each point of the configuration space $\left(\mathbb{R}^{2}\right)$. The scheme is defined such that the hue is correlated with the complex phase $\arg \left(\phi^{1}+\mathrm{i} \phi^{2}\right)$ and the 

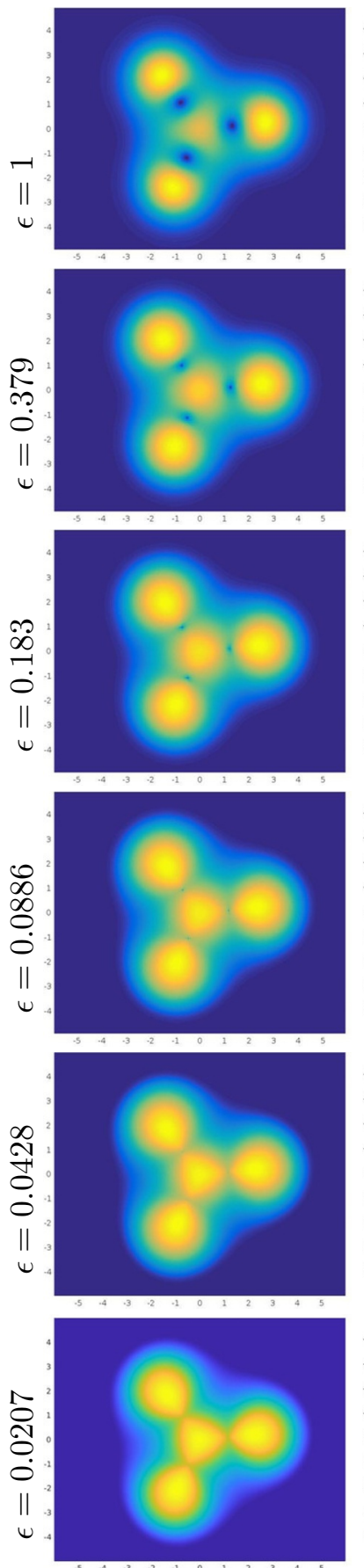
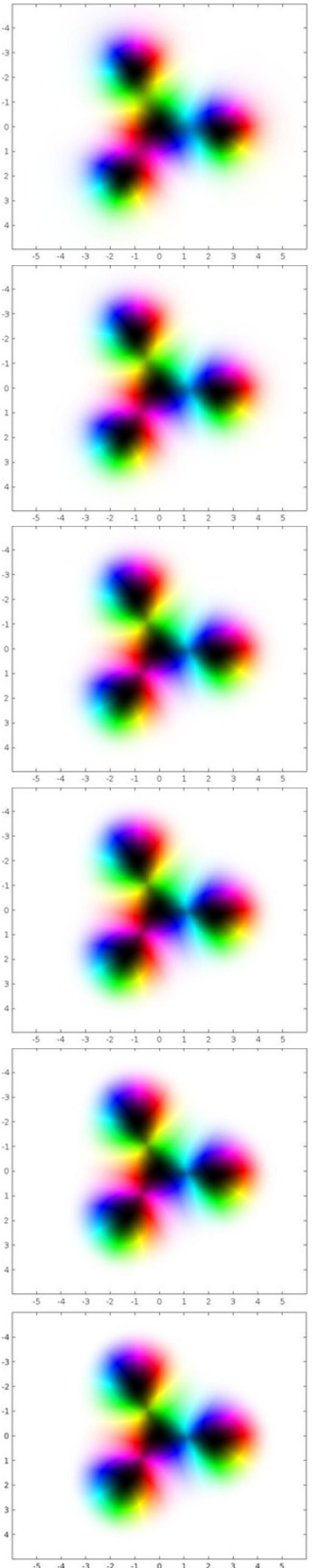
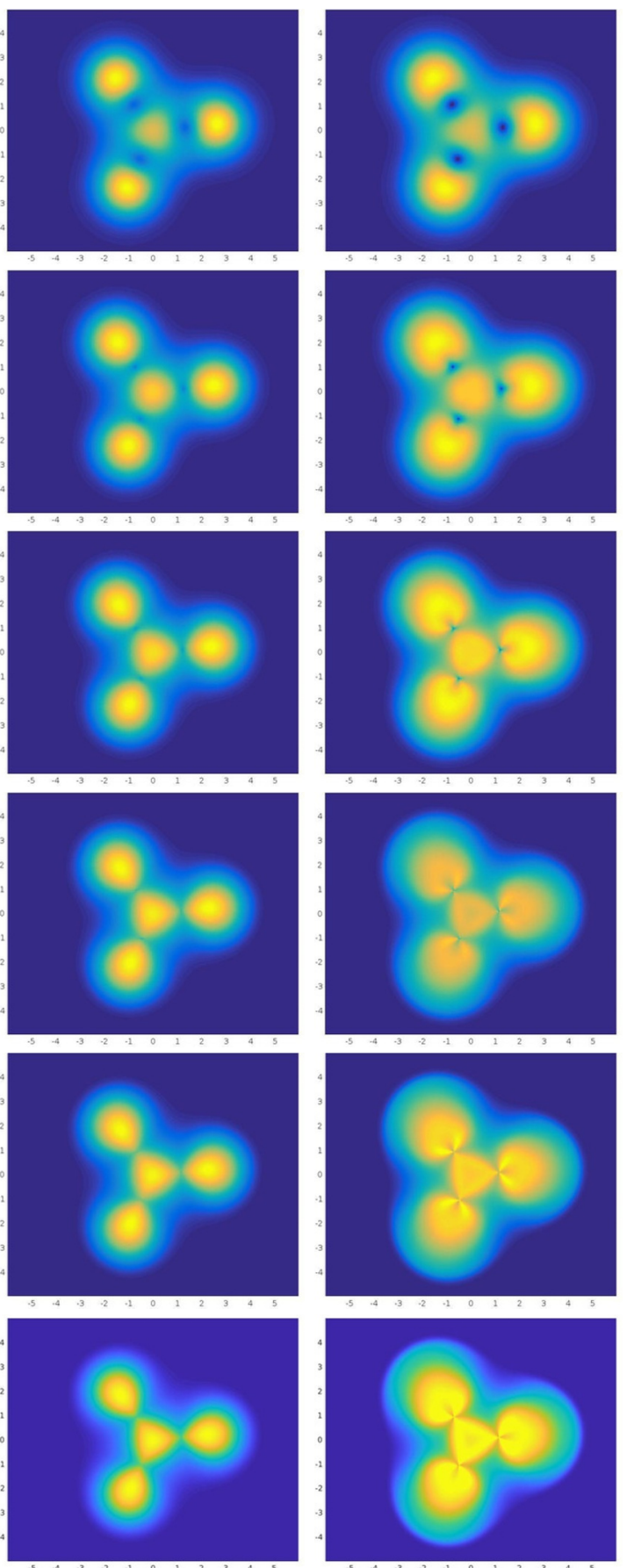

Figure 6. The $Q=1+1+1+1$ tetrahedral baby-Skyrmion solution as a function of $\epsilon$. The columns display the topological charge density, the field orientation using the color scheme described in the text, the total energy and finally the kinetic term $-\epsilon \mathcal{L}_{2}$. We note that the tetrahedrally symmetric baby Skyrmion exists also for large $\epsilon$ (i.e. $\epsilon=1)$. 

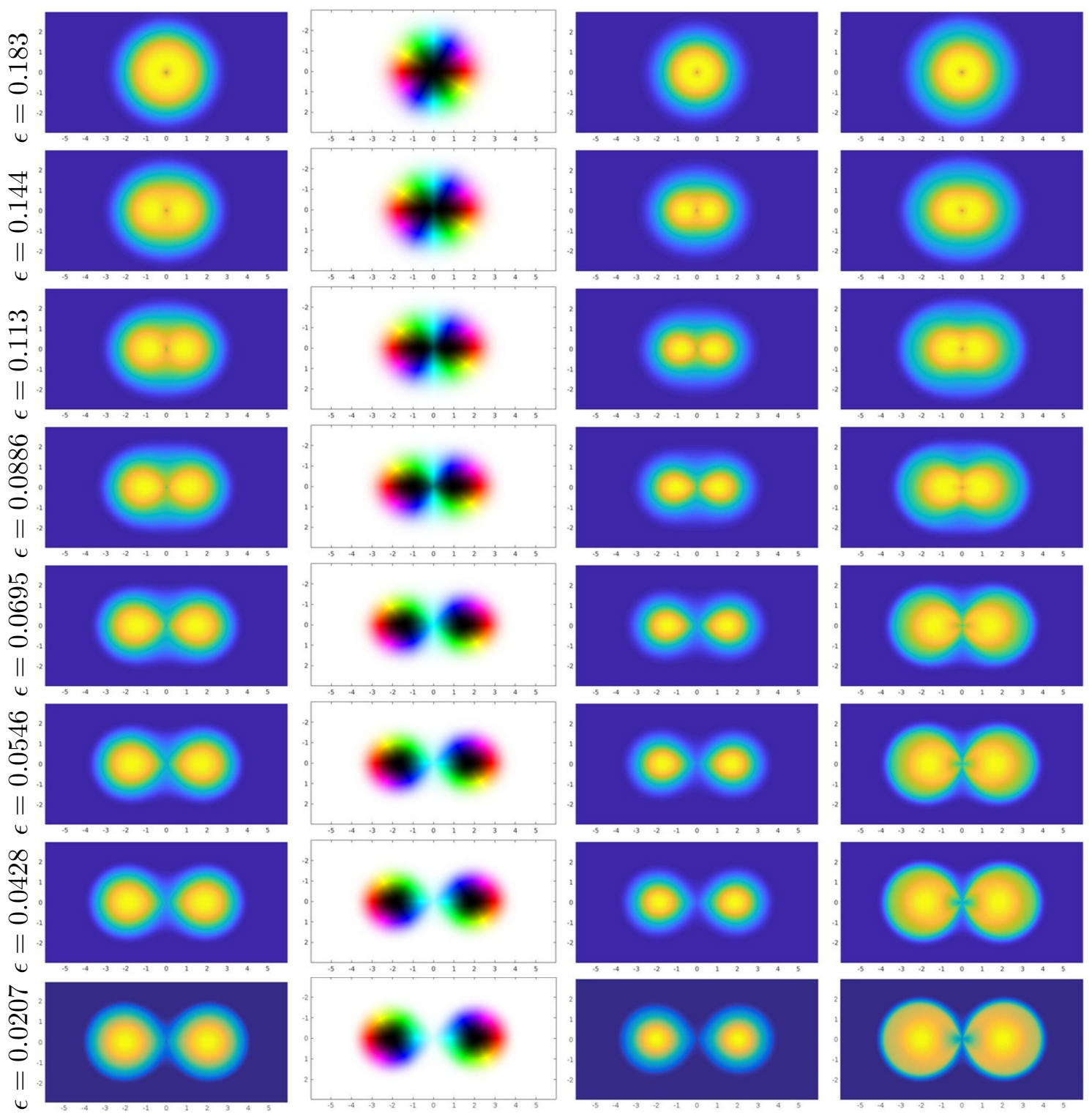

Figure 7. The $Q=1+1$ baby-Skyrmion solution as a function of $\epsilon$. The columns display the topological charge density, the field orientation using the color scheme described in the text, the total energy and finally the kinetic term $-\epsilon \mathcal{L}_{2}$.

lightness is determined by the value of $\phi^{3}$. Explicitly, $\phi^{3}=1$ is white, $\phi^{3}=-1$ is black, $\arg \left(\phi^{1}+\mathrm{i} \phi^{2}\right)=0$ is red, $\arg \left(\phi^{1}+\mathrm{i} \phi^{2}\right)=2 \pi / 3$ is green and $\arg \left(\phi^{1}+\mathrm{i} \phi^{2}\right)=4 \pi / 3$ is blue.

In particular, we find that the two $N=2$ baby Skyrmions next to each other is always stable - even for $\epsilon=1$. What happens when $\epsilon$ is decreased, is that the hole in each $N=2$ soliton is shrunk to a point and asymptotically, the two Skyrmions become axially symmetric - almost unaware of each others presence. Notice that the orientation of the two baby Skyrmions is always in the attractive channel, i.e. such that e.g. two patches of red touch each other, see the second column of figure 4. At larger values of $\epsilon$, the two $N=2$ solitons are elongated along the axis joining their centers. This can be interpreted 

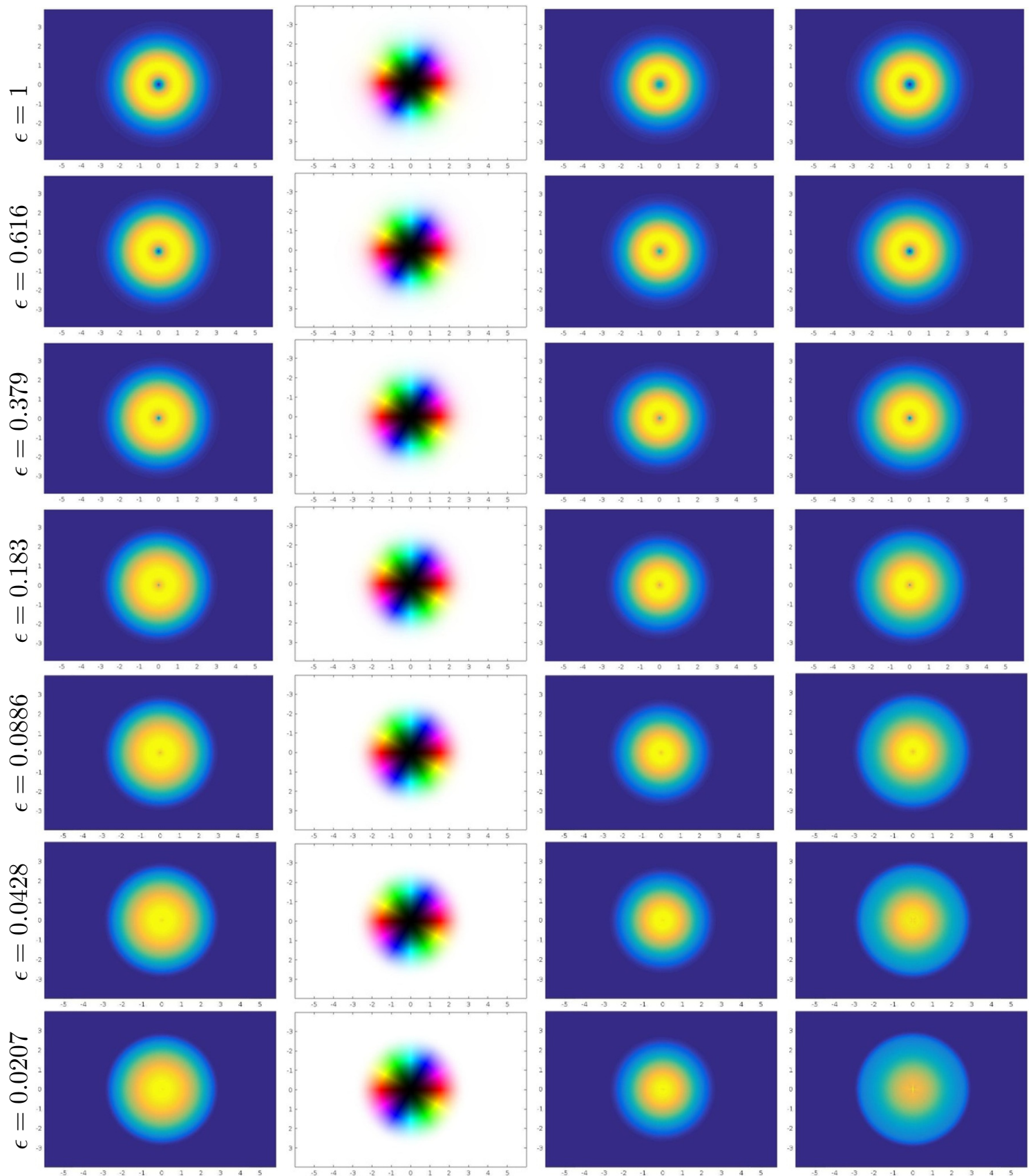

Figure 8. The $N=2$ baby-Skyrmion solution as a function of $\epsilon$, which is the stable $Q=2$ soliton. The columns display the topological charge density, the field orientation using the color scheme described in the text, the total energy and finally the kinetic term $-\epsilon \mathcal{L}_{2}$. 

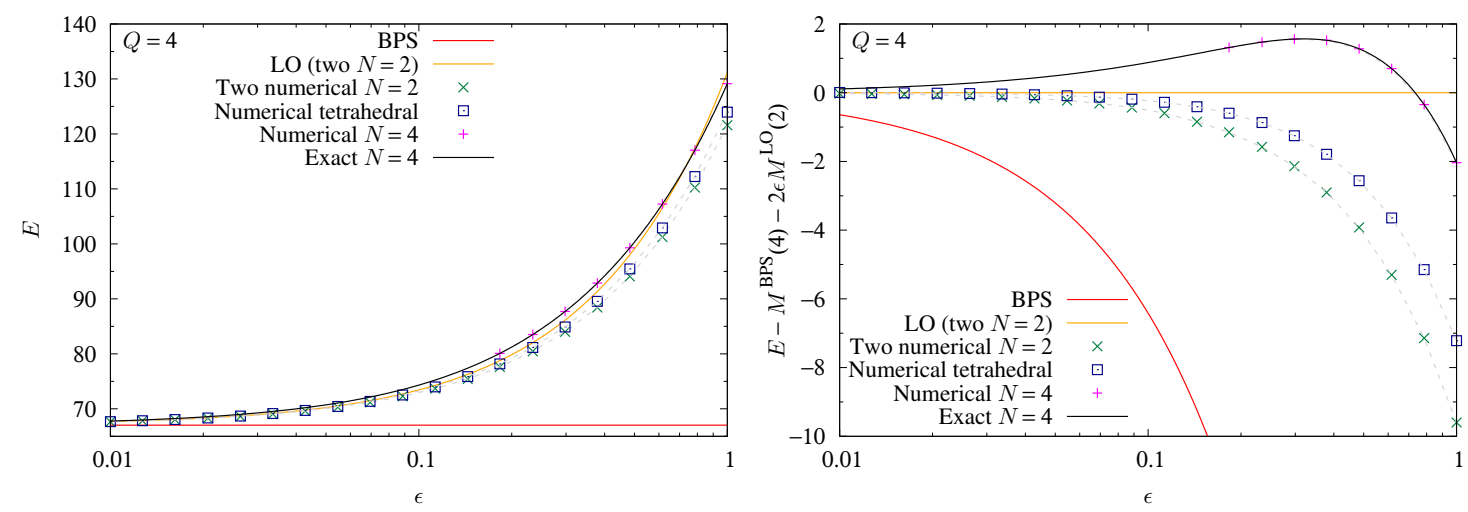

Figure 9. The total energies of the baby-Skyrmion solutions in the $Q=4$ sector. The red line is the BPS bound, the orange curve the LO correction (coming from the kinetic term) for two $N=2$ Skyrmions. The numerical solutions are: the two $N=2$ Skyrmions side-by-side (green xs), the tetrahedral Skyrmion (blue squares with a dot in the middle), and the axially symmetric $N=4$ Skyrmion (magenta pluses). Finally, the latter numerical $N=4$ solution is compared to the exact solution (using the 1-dimensional ODE). For clarity, the right-hand side panel shows the same data relative to the orange line, being the LO correction. At $\epsilon \lesssim 0.15$ the axially symmetric soliton becomes unstable and decays to the tetrahedral one - which is only metastable.
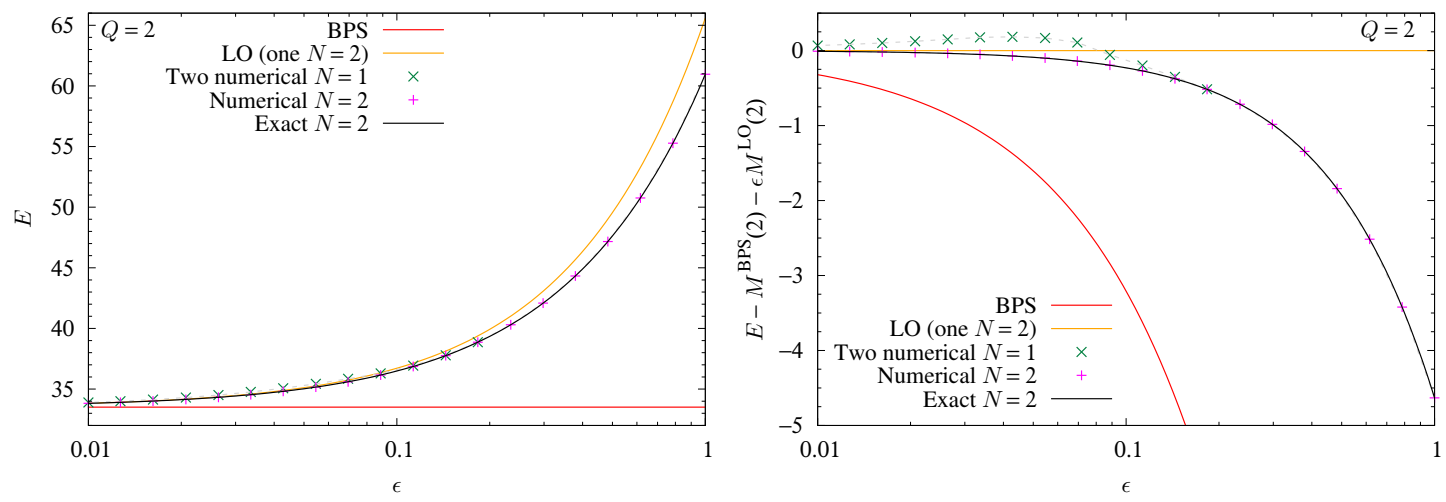

Figure 10. The total energies of the baby-Skyrmion solutions in the $Q=2$ sector. The red line is the BPS bound, the orange curve the LO correction (coming from the kinetic term) for a single $N=2$ Skyrmion. The numerical solutions are: the two $N=1$ Skyrmions side-by-side (green xs), and the axially symmetric $N=2$ Skyrmion (magenta pluses). Finally, the latter numerical $N=2$ solution is compared to the exact solution (using the 1-dimensional ODE). For clarity, the right-hand side panel shows the same data relative to the orange line, being the LO correction. At $\epsilon \gtrsim 0.15$ the two $N=1$ 's side-by-side become unstable and decay to the axially symmetric $N=2$. 
as an effect due to the strong binding force between them. For small $\epsilon$, this effect goes away and the binding energy is also drastically reduced. We will discuss the binding energies separately in the next section.

Baby-Skyrmion solitons are free to deform their shape and are able to split up to the lowest energy state. Nevertheless, many local minima of the energy functional exist and they correspond to metastable states. In order to know which configurations are the stable ones, we start with many different initial guesses and see what they converge to by the numerical flow.

We expect from the fact that $N_{\star} \approx 2$, that the $N=4$ axially symmetric soliton will be only metastable. As an explicit check, we perform the numerical calculations which are shown in figure 5. Perhaps surprisingly, it turns from metastable at $\epsilon \approx 1$ into unstable around $\epsilon \approx 0.15$, see the last row of figure 5. The numerical algorithm finds a lower-energy configuration, which is composed by four $N=1$ Skyrmions attached to each other in a tetrahedral arrangement. This is somewhat surprising, because as we shall see shortly, the $N=1$ soliton is actually not stable for $\epsilon=0.15$ unless it is alone. Once it is near another $N=1$ soliton, they will merge into a deformed $N=2$ soliton at that $\epsilon$. The high degree of discrete symmetry — tetrahedral symmetry — somehow prevents that from happening.

There are two possibilities: either there is a crossover, so that the tetrahedral configuration becomes stable and the $N=4$ axially symmetric soliton becomes unstable around $\epsilon=0.15$ or both the tetrahedral and axially symmetric solitons exist for $\epsilon \gtrsim 0.15$ and the axially symmetric solution simply becomes unstable around $\epsilon=0.15$ such that the decay to the nearest (in energy) metastable state is the tetrahedral baby Skyrmion. In order to determine the phase diagram of the $Q=4$ sector, we need to know the energies of the tetrahedral soliton also for larger values of $\epsilon$. Thus we use the tetrahedral configuration as a seed (initial guess) in the calculation and calculate its energy for larger $\epsilon$ all the way to $\epsilon=1$, see figure 6. This reveals that the tetrahedral $Q=4$ baby Skyrmion exists also for large values of $\epsilon$ (even though two $N=1$ baby-Skyrmions next two each other are unstable, see below).

Then to determine the phase diagram of the $Q=4$, we plot the total energies of the different solutions in figure 9. From the figure - especially the right-hand side panel we can see that the $N=4$ axially symmetric configuration is metastable for $\epsilon \gtrsim 0.15$ with the highest energy. The tetrahedral soliton is metastable with an intermediate energy, but not too far above the lowest-energy state. Finally, the two $N=2$ Skyrmions side-by-side is the most energetically favorable solution in the entire range of the $\epsilon$ considered here.

For $\epsilon=0.0207$ it is rather convincing that the $\epsilon \rightarrow 0$ limit turns the solitons into a lattice of axially symmetric solutions sitting next to each other with the most stable axially symmetric component being the $N=2$ baby Skyrmion. The contact to neighboring solitons becomes point-like in the $\epsilon \rightarrow 0$ limit. This is consistent with the restricted harmonic property that the near-BPS (baby) Skyrmions should possess.

We will now turn to the $Q=2$ sector. Again, as $N_{\star} \approx 2$, we know that the $N=1$ axially symmetric baby Skyrmion is stable only in isolation. When exposed to more baby Skyrmions, it has to be either metastable or unstable; that is, it should be energetically favorable to combine into $N=2$ Skyrmions. It turns out that for $\epsilon=1$, two $N=1$ axially symmetric baby Skyrmions next to each other are unstable and they immediately combine 
into a single $N=2$ soliton with axial symmetry [34]. However, for small enough $\epsilon$, the two $N=1$ 's side-by-side become metastable, see figure 7 . The critical $\epsilon_{2}$ where the baby Skyrmion departs from axial symmetry is around $\epsilon \approx 0.2$, whereas the critical $\epsilon_{1}$ where the baby Skyrmion is clearly composed of two individual solitons is around $\epsilon_{1} \approx 0.1$. For $\epsilon<0.06$ the two individual solitons are almost only connected by a "single point."

As a check on our numerical calculations, we compute the $N=2$ axially symmetric baby Skyrmions as well, see figure 8 . The figure shows that the hole in the middle of the soliton is shrunk to a point in the limit of $\epsilon \rightarrow 0$.

To check how these solutions fit into our picture, we show their total energies in figure 10. We can thus confirm that the phase diagram in the $Q=2$ sector is determined by the LO correction to the energy, which makes the two $N=1$ baby Skyrmions sitting side-by-side metastable for $\epsilon \lesssim 0.15$ and unstable above. We can also confirm by comparing the 2-dimensional PDE calculations with the 1-dimensional ODE ones (for the $N=2$ case), that our numerical accuracy is incredibly good for the entire range of $\epsilon \mathrm{s}$ considered here.

In order to complete our perturbative scheme, we need to calculate the binding energies between two Skyrmions, which will be the topic of the next section.

\section{$5 \quad$ Binding energies}

In this section we will discuss the binding energies of two $N=2$ baby Skyrmions sitting side-by-side and, in the last part, also the case of two $N=1$ side-by-side. We have performed very high-resolution numerical calculations that give us the answers we seek for the baby Skyrmion case. However, for the 3-dimensional Skyrmions, the numerical calculations become much more difficult - even with the utilization of adaptive methods. Therefore we want to push the perturbative approach to capture the physics and respective energies of the composite near-BPS solitons.

Since we have the compacton solutions, the background is analytically known. The difficulty is to impose the boundary or cusp condition (3.90) that the derivative of the perturbation fields obey at the boundary of the compacton, but this can easily be done in polar coordinates. Furthermore, the composite configuration of two baby Skyrmions side-by-side will need a gluing condition, which we shall discuss shortly. Finally, the perturbations must go to zero at spatial infinity, which is the easiest condition to impose.

In section 3.2 we showed that the restricted harmonic map for the $Q=2+2$ configuration consists of two separated axially symmetric compactons with random relative orientation. However, if for a single baby Skyrmion the problem of the background solution is solved by the restricted harmonic condition, for the multi-soliton case further considerations are needed. To understand the reason for this difference, we must consider the physical meaning of the zeroth-order background field. This field represents the limit of the exact solution $\phi$ of the Lagrangian (2.1) when $\epsilon$ tends to zero, i.e. the final configuration that we obtain if we adiabatically switch off the interaction among the solitons. The static solution $\phi$ exists only if the baby Skyrmions interact with an attractive force that depends on the relative orientation among them [12]. Therefore, we expect that even in the limit of vanishing interaction $(\epsilon \rightarrow 0)$ the final configuration will conserve a particular 
relative orientation. Then, we conclude that for the near-BPS multi-baby Skyrmion case, the restricted harmonic condition is not sufficient to identify the correct zeroth-order solution since it does not yield any restriction on the relative orientation (or distance) among axially symmetric compactons.

In order to fix the background configuration among all the restricted harmonic maps, we propose to identify the right map by looking at the form of the interaction between two well-separated baby Skyrmions. The potential $V$ among these solitons has been calculated in ref. [12] for the baby-Skyrme model (the same Lagrangian (2.1) with $\epsilon=1$ ) and for the $Q=2+2$ case it takes the form

$$
V_{2+2} \propto-\cos (\alpha-\beta) \frac{e^{-m R}}{\sqrt{m R}}
$$

where $R$ is the distance between the soliton centers, $\alpha$ and $\beta$ are the two respective phases and $m$ is the (perturbative) pion mass. From this expression we recognize that, for fixed distance $R$, the minimum of the potential is obtained for $\alpha-\beta=0$, i.e. when the two baby Skyrmions have the same orientation. Therefore, even if the form of the potential (5.1) holds only at large distances, we guess that in the limit of vanishing interaction (when $\epsilon \rightarrow 0)$ the two solitons keep their relative orientation unchanged.

Instead, for what concerns the relative distance, we expect that in the limit of vanishing interaction, the two baby Skyrmions must flow to a configuration in which they overlap their tails less and less. However, since the tail of a near-BPS baby Skyrmion vanishes rapidly with $\epsilon$ as is clear from eq. (3.52), the two solitons flow to a distance that is very close to the sum of their radii.

Summarizing, we guess that in the limit $\epsilon \rightarrow 0$, the background solution for the $Q=$ $2+2$ configuration is given by two axially symmetric compactons with the same orientation touching each other at a single point. This guess seems to be confirmed by numerical calculations in figure 4 and we will thus use it in the following.

Since the zeroth-order configuration for the two compactons is such that the other baby Skyrmion is a copy of itself, spatially translated by a distance of its own diameter (roughly), it is not necessary to calculate the perturbation over the entire plane, but we can reduce the problem by identifying appropriate boundary conditions for a field living in an appropriate half of $\mathbb{R}^{2}$.

The boundary conditions are sketched in figure 11. In addition to setting the perturbation to zero at $r=0$ and imposing the condition (3.90), we need to impose the following boundary (gluing) conditions

$$
\begin{aligned}
\partial_{x} \delta \phi^{1}(0, y) & =0, \\
\delta \phi^{2}(0, y) & =0, \\
\partial_{x} \delta \phi^{3}(0, y) & =0,
\end{aligned}
$$

which ensure that the soliton can be glued together with its partner. The second boundary condition is "odd" because this field is odd under parity transformations and the only way to connect the perturbation to a field that is not flipped in $x \rightarrow-x$ (because that 


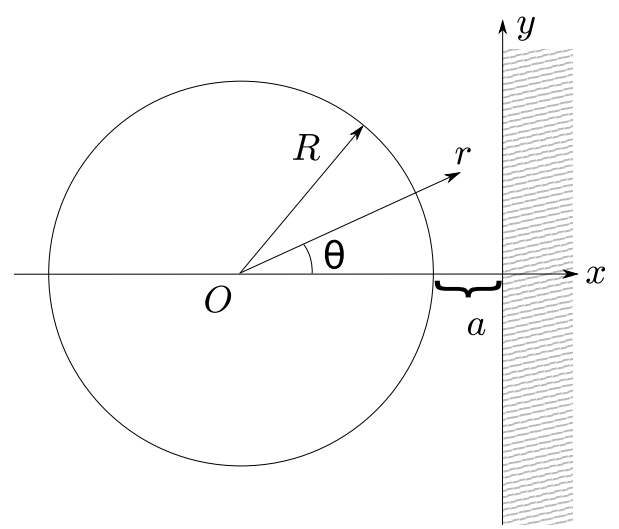

Figure 11. Coordinate system $(r, \theta)$ for perturbation of the two $N=2$ baby Skyrmions sitting side-by-side. Boundary conditions need to be imposed at $O$ and at the compacton boundary $r=R$ as well as at $x=0$ (at $r e^{i \theta}=R+a+i y$ ) for attaining the ability of gluing the two solitons together.

would make it an anti-Skyrmion) is to impose the boundary condition as given in the above equations. Because the condition (3.90) is easier in polar coordinates, we prefer to impose the $x=0$ gluing conditions on the equation in polar coordinates.

In terms of the perturbation fields given in eq. (3.70), we can write the boundary conditions as

$$
\begin{aligned}
\delta f(0, \theta) & =0, & \delta \theta(0, \theta) & =0, \\
\delta f(\infty, \theta) & =0, & \delta \theta(\infty, \theta) & =0, \\
\delta f(r, 0) & =\delta f(r, 2 \pi), & \delta f_{r}(r, 0) & =\delta f_{r}(r, 2 \pi), \\
\delta \theta(r, 0) & =\delta \theta(r, 2 \pi), & \delta \theta_{r}(r, 0) & =\delta \theta_{r}(r, 2 \pi),
\end{aligned}
$$

while at $r=R$ we have

$$
\delta f_{r}\left(R^{-}, \theta\right)-\delta f_{r}\left(R^{+}, \theta\right)=\frac{4}{R},
$$

and finally at $x=0$ - which is $r e^{i \theta}=R+a+i y$ in polar coordinates — the boundary conditions (5.2)-(5.4) become

$$
\begin{aligned}
\delta \theta & =-\tan (N \theta) \delta f, \\
\partial_{x}\left(\delta \theta^{2}+\delta f^{2}\right) & =0,
\end{aligned}
$$

and the latter equation can be written as

$$
\begin{aligned}
\delta f & =N^{-1} \cot (N \theta)\left(r \cot (\theta) \delta f_{r}-\delta f_{\theta}\right), & & \theta>0, \\
\delta f_{r} & =\delta f_{\theta}=0, & & \theta=0 .
\end{aligned}
$$

Figure 12 shows the boundary conditions for the lattice problem (the discretized PDEs) at the $x=0$ boundary. The lattice point $(I+1, j)$ is determined by the condition (5.9) 

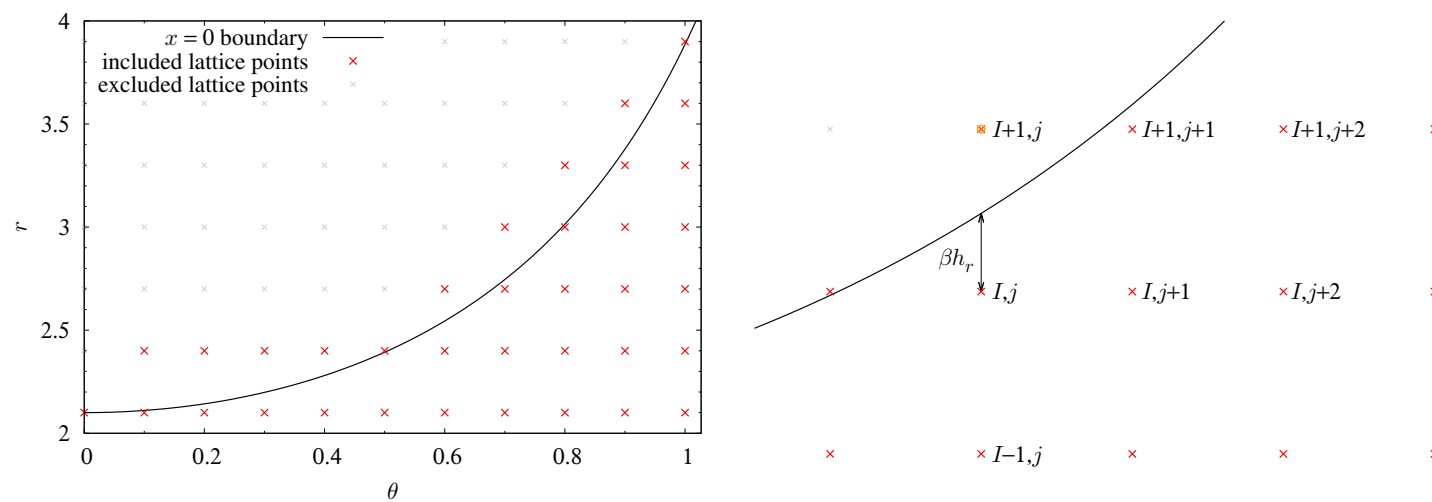

Figure 12. Boundary (gluing) conditions at $x=0$ for the discretized (lattice) problem. This example is shown for $N=1, m=1$ and $a=0.1$ with an exaggeratedly big step size of 0.3 chosen for illustrative purposes. The right-hand panel shows a zoom with the lattice coordinates used in eq. (5.11).

which is written in discretized form as

$$
\begin{aligned}
& \delta f^{I+1, j}\left(\beta-\frac{\cot \left(N \theta^{j}\right)}{N}\left[\left(r^{I}+\beta h_{r}\right) \cot \left(\theta^{j}\right) \frac{(2 \beta+1)}{2 h_{r}}+\frac{3 \beta}{2 h_{\theta}}\right]\right) \\
& =(\beta-1) \delta f^{I, j}+\frac{\cot \left(N \theta^{j}\right)}{N}\left[\left(r^{I}+\beta h_{r}\right) \cot \left(\theta^{j}\right) \frac{-4 \beta \delta f^{I, j}+(2 \beta-1) \delta f^{I-1, j}}{2 h_{r}}\right. \\
& \left.-(1-\beta) \frac{-3 \delta f^{I, j}+4 \delta f^{I, j+1}-\delta f^{I, j+2}}{2 h_{\theta}}-\beta \frac{4 \delta f^{I+1, j+1}-\delta f^{I+1, j+2}}{2 h_{\theta}}\right],
\end{aligned}
$$

with $\beta$ the offset of the $x=0$ boundary from the second-last lattice point in the calculation.

It will be instructive to write the perturbations $\delta f, \delta \theta$ as follows

$$
\begin{aligned}
\phi= & \boldsymbol{\varphi}+\boldsymbol{\delta} \phi \\
= & \boldsymbol{\varphi}+\boldsymbol{\Delta}_{\perp} \times \boldsymbol{\varphi}+\frac{1}{2} \boldsymbol{\Delta}_{\perp} \times\left(\boldsymbol{\Delta}_{\perp} \times \varphi\right) \\
= & \left(\begin{array}{c}
\sin f \cos N \theta \\
\sin f \sin N \theta \\
\cos f
\end{array}\right)+\left(\begin{array}{c}
\cos f \cos N \theta \\
\cos f \sin N \theta \\
-\sin f
\end{array}\right) \delta f \\
& +\left(\begin{array}{c}
-\sin N \theta \\
\cos N \theta \\
0
\end{array}\right) \delta \theta-\left(\begin{array}{c}
\sin f \cos N \theta \\
\sin f \sin N \theta \\
\cos f
\end{array}\right) \frac{\delta f^{2}+\delta \theta^{2}}{2} \\
= & \left(\begin{array}{c}
\sin (f+\delta f) \cos (N \theta+\delta \theta) \\
\sin (f+\delta f) \sin (N \theta+\delta \theta) \\
\cos (f+\delta f)
\end{array}\right)+\mathcal{O}\left(\delta \theta^{2}, \delta f \delta \theta, \delta f^{3}\right),
\end{aligned}
$$

where we have used eq. (3.70).

We now turn to solving the coupled PDEs (3.72) with the boundary conditions (5.5) and (5.9). As a good starting point, we take the perturbation of the axially symmetric 


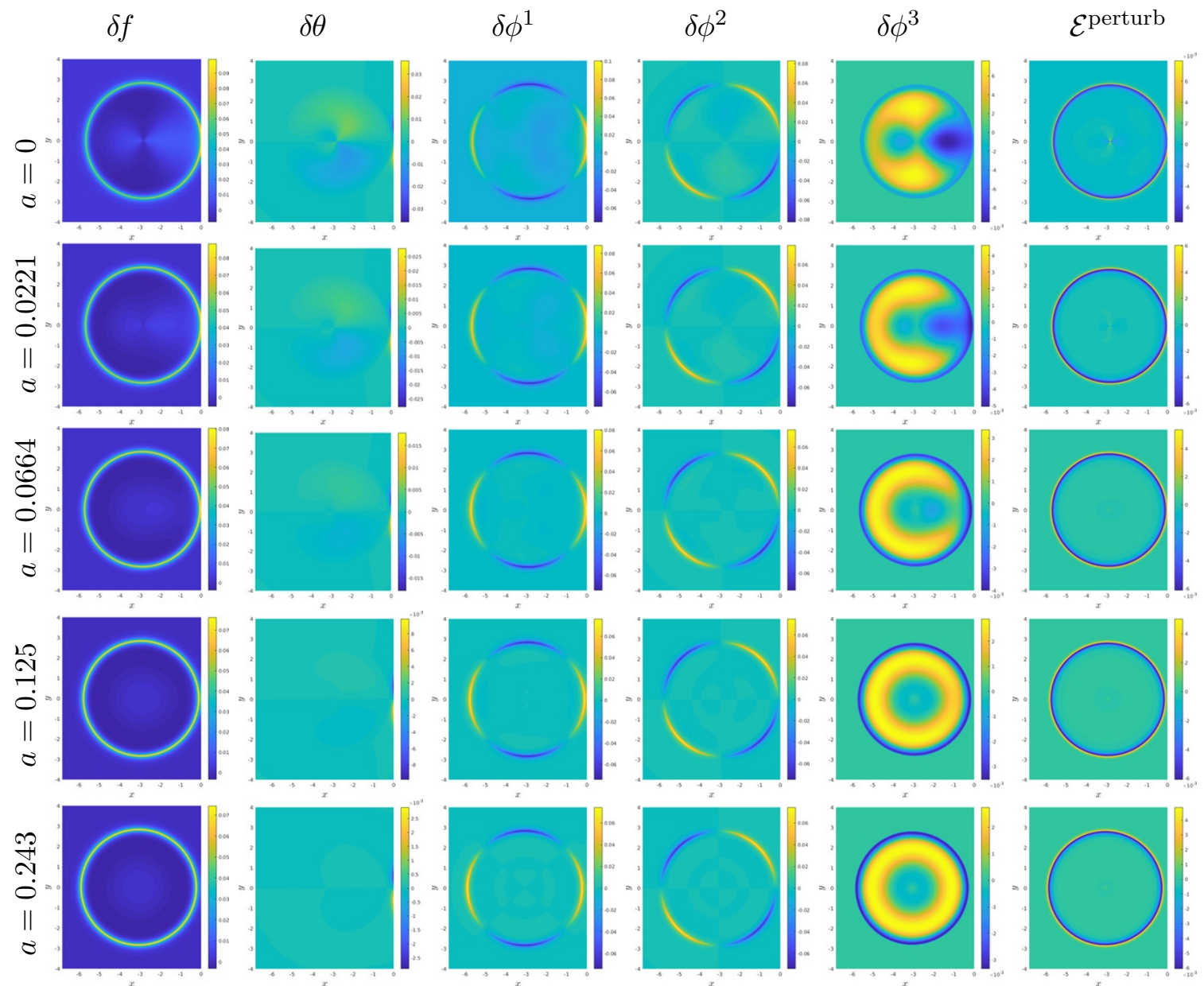

Figure 13. Numerical solution for $\delta f$ and $\delta \theta$ for two $N=2$ compactons situated next two each other with separation distance $2 a$ for $\epsilon=0.01$. Due to symmetry of the problem, only the left-hand side is calculated and shown. The gluing conditions (5.9) are imposed at $x=0$, which is located at the right-most part of every panel. Each row displays the perturbations $\delta f, \delta \theta, \delta \phi^{1}, \delta \phi^{2}, \delta \phi^{3}$ and the energy density of the perturbation $\mathcal{E}^{\text {perturb }}$, each in its column. Each panel is cropped so as to render the content as clear as possible; the left, top and bottom border do not represent the size of the simulations.

compacton for given $\epsilon$ and $N$ as the initial condition. What the PDE problem then boils down to, is to implement the gluing conditions (5.9) at $x=0$ (i.e. midway between the two compactons). The gluing conditions break the axial symmetry which provides nontrivial $\theta$ dependence for $\delta f$ which in turn acts as a source for $\delta \theta$.

Figures 13 and 14 display the solutions for the perturbations $\delta f$ and $\delta \theta$ for $N=2$ and $\epsilon=0.01,0.0428$, respectively. Due to the nontrivial relationship between the perturbations $\delta f, \delta \theta$ and the vector perturbations $\delta \phi$ of eq. (5.12), we show the three vector components in columns 3 through 5 of the figures 13 and 14 . Finally, the last and sixth column of the latter figures shows the energy density (3.66) of the perturbation to $\mathrm{N}^{2} \mathrm{LO}$, i.e. to the third order in $\epsilon$. The radial perturbation, $\delta f$, continues to do the job of obeying the 


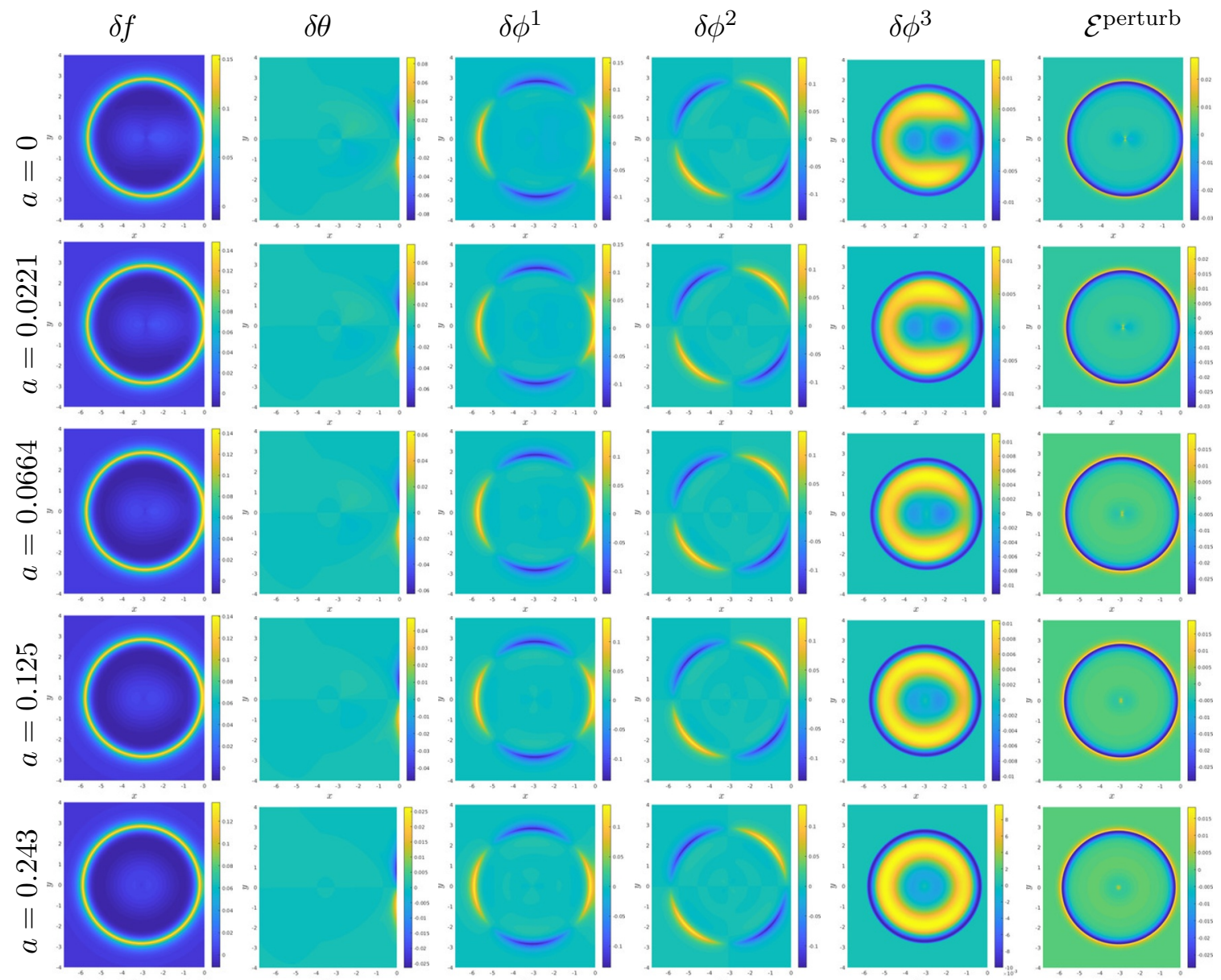

Figure 14. Numerical solution for $\delta f$ and $\delta \theta$ for two $N=2$ compactons situated next two each other with separation distance $2 a$ for $\epsilon=0.0428$. Due to symmetry of the problem, only the lefthand side is calculated and shown. The gluing conditions (5.9) are imposed at $x=0$, which is located at the right-most part of every panel. Each row displays the perturbations $\delta f, \delta \theta, \delta \phi^{1}, \delta \phi^{2}$, $\delta \phi^{3}$ and the energy density of the perturbation $\mathcal{E}^{\text {perturb }}$, each in its column. Each panel is cropped so as to render the content as clear as possible; the left, top and bottom border do not represent the size of the simulations.

cusp condition (5.6), which is seen in the figures as a yellow ring (the maximum) at the compacton boundary. The only $\theta$-dependence is induced by the gluing condition (5.9) near the right-hand side boundary of each panel; the condition on $\delta f$ is slightly cumbersome as $\delta f^{2} / \cos ^{2}(N \theta)$ must obey the Neumann boundary condition at $x=0$. The effect is most visible for small values of $a$ and as soon as $a$ is of order of the thickness of the "yellow" ring, which is the perturbation deformation due to the cusp condition, the $\theta$-dependence is almost negligible. This can also be seen in the second column of the figures, where a nontrivial solution for $\delta \theta$ is most pronounced for $a=0$. The $\theta$-dependence in $\delta \theta$ at the $x=0$ boundary is visible as positive (negative) values for $y$ negative (positive). This effect persists when $a$ is increased, but the magnitude of the values of $\delta \theta$ at the $x=0$ boundary become exponentially suppressed in line with the tail of the perturbation around 
the compacton does. The fact that the solutions are $N=2$ baby Skyrmions is clearly visible in columns 3 and 4 , where the cusp in $\delta f$ switches sign four times as one goes around the compacton border. The induced $\theta$-dependence is also very clear for $a=0$ and $a=0.0221$ in column 5 in the third component of the vector perturbation, $\delta \phi^{3}$. The last column in the two figures shows the energy density of the perturbation and it is not easy to see the result of the integration with the naked eye. In fact, the largest effect of the gluing is geometric, meaning that cutting part of the tail of the perturbation at $x=0$, diminishes the contribution to the energy. One could anticipate that the gluing of the two compactons would give a positive contribution counteracting the geometric decrease in the perturbation energy; although that happens for the field $\delta f$, the induction of $\theta$ dependence turns on nontrivial behavior for $\delta \theta$ which lowers the energy slightly. At the distance $a=0.243$, there is almost no visible effect of the gluing conditions and the energy is very close to the sum of two compactons. Figure 13 shows the case for very small $\epsilon=0.01$ and in figure $14 \epsilon=0.0428$ is slightly larger. The effect is simply an amplification of perturbations and in particular, the thickness of the perturbation in $\delta f$ due to the cusp condition is far larger for $\epsilon=0.0428$ than for $\epsilon=0.01$.

Figure 15 shows the perturbative contribution to the energy at next-to-next-to-leading order ( $\mathrm{N}^{2} \mathrm{LO}$ ) for two $N=2$ baby Skyrmions as function of the separation distance $2 a$, see figure 12. The four panels show $\epsilon=0.01, \epsilon=0.0207, \epsilon=0.0428$ and $\epsilon=0.0886$, respectively and for all panels, the smallest energy (meaning the largest negative contribution to the energy) is at $a=0$, which means when the two compactons exactly touch each other at a point. For the three smallest values of $\epsilon$ (i.e. $\epsilon=0.01,0.0207,0.0428$ ), the perturbation energy turns out to be lower than the geometric energy for small $a$ (and equal at large $a$ ). The geometric energy is simply the perturbation energy of the axially symmetric compacton cut off at $x=0$. However, for $\epsilon=0.0886$ the perturbation energy crosses over the geometric line at $a \sim 0.12$ and is slightly above the geometric one at $a=0$, indicating that the gluing condition builds up some tension or excess energy in the perturbation for this "large" value of $\epsilon$. Of course, our perturbation scheme is best trusted at small $\epsilon$, so this may well be an indication of the approximation starting to deteriorate.

We finally, compare the $\mathrm{N}^{2} \mathrm{LO}$ result of the perturbation theory of the energy of two $N=2$ baby Skyrmions sitting side-by-side - taking into account the binding energy with the full numerical (brute-force) PDE calculations, see figure 16. The two results are incredibly close to each other, in particular for small values of $\epsilon$. Since the binding energy requires a PDE calculation as well, it is not clear which result is more accurate. We expect the perturbative result to be most accurate of the two for small $\epsilon \sim 0.01$, whereas the full numerical PDE calculations are most accurate for large values of $\epsilon \sim 0.1$.

Before summarizing the results for the binding energies, we will repeat the perturbative $\mathrm{N}^{2} \mathrm{LO}$ calculation of the two baby Skyrmions side-by-side, but this time for $N=1$ compactons. This solution is only metastable for small $\epsilon$ while for $\epsilon \gtrsim 0.15$ it is unstable. Nevertheless, since we have performed the full PDE computations of these composite baby Skyrmions in section 4 , it is a good test of the perturbative scheme.

Similarly to the case of $Q=2+2$, for the case of two $N=1$ baby Skyrmions side-by-side, we must specify the background solution around which we calculate the per- 


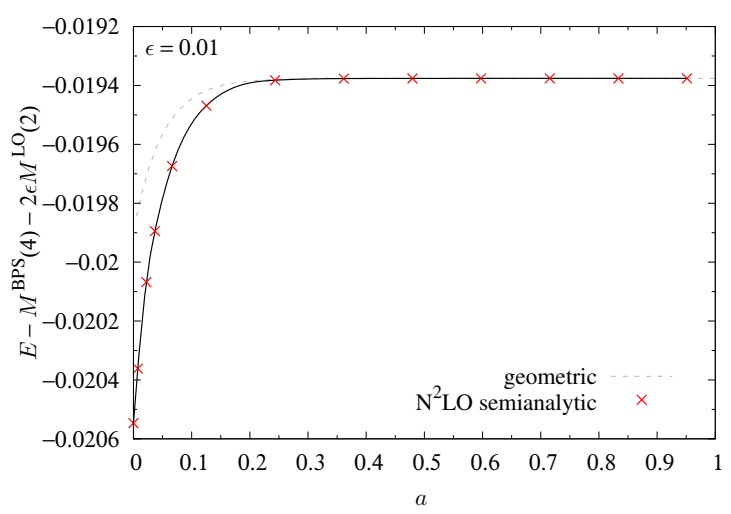

(a)

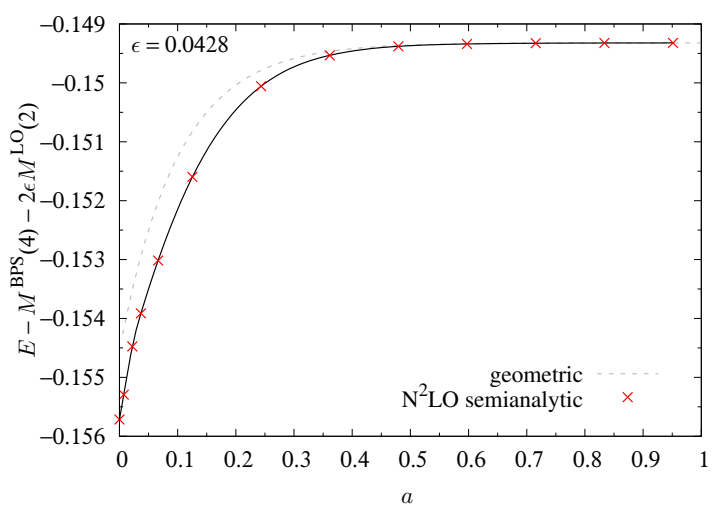

(c)

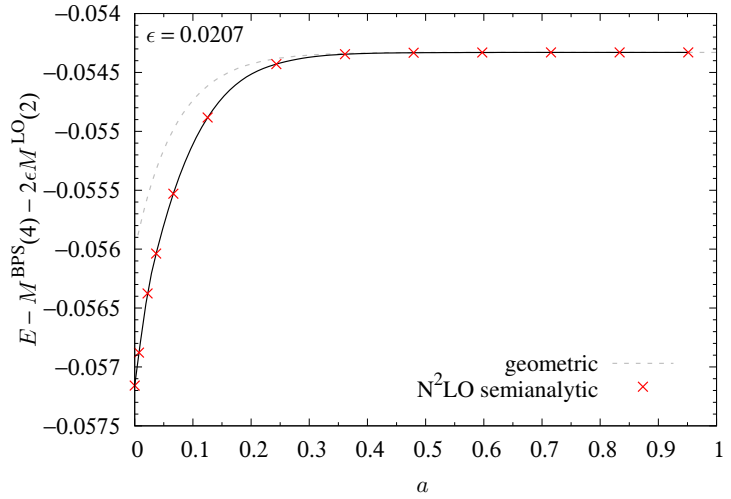

(b)

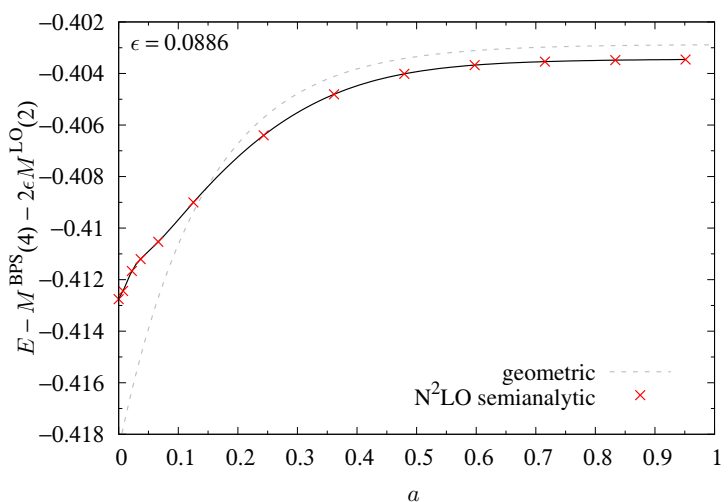

(d)

Figure 15. The $\mathrm{N}^{2} \mathrm{LO}$ corrections to the energy for two $N=2$ baby Skyrmions as a function of the separation distance $2 a$, see figure 12. The panels correspond to different values of $\epsilon$ : (a) $\epsilon=0.01$, (b) $\epsilon=0.0207$, (c) $\epsilon=0.0428$ and (d) $\epsilon=0.0886$. The gray-dashed line is calculated as the geometric cut at $x=0$ of the spherically symmetric compacton energy. The red crosses show the results of the $\mathrm{N}^{2} \mathrm{LO}$ contribution to the energy from the PDE calculation of $\delta f$ and $\delta \theta$. The black line is a cubic interpolation between the PDE results. For $\epsilon=0.0886$ (d) the $\mathrm{N}^{2} \mathrm{LO}$ perturbation energy crosses over the geometric estimate and hence the excess energy starts to build up at this "large" value of $\epsilon$ for $N=2$.

turbation (5.12). Again the restricted harmonic condition does not identify uniquely the right compacton configuration that is needed since it does not specify any condition on the relative orientation (or relative distance). Following the discussion of the $Q=2+2$ case, we look at the long-range potential of two well-separated $N=1$ baby Skyrmions [12]:

$$
V_{1+1} \propto \cos (\alpha-\beta) \frac{e^{-m R}}{\sqrt{m R}}
$$

where again $R$ is the relative distance, $\alpha$ and $\beta$ are the two respective phases and $m$ is the pion mass. The potential (5.14) generates the maximum attractive force in case of two baby Skyrmions with opposite orientation, i.e. $\alpha=\beta+\pi$. Therefore, in contrast to the $Q=2+2$ case, among all the axially symmetric compactons side-by-side, we choose 


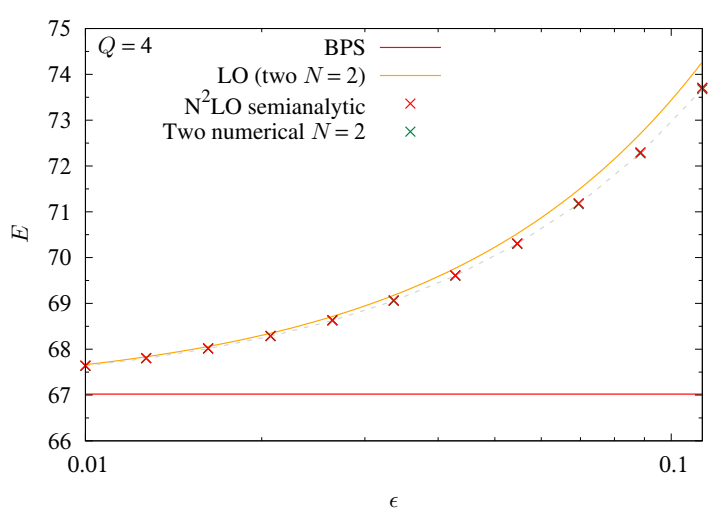

(a)

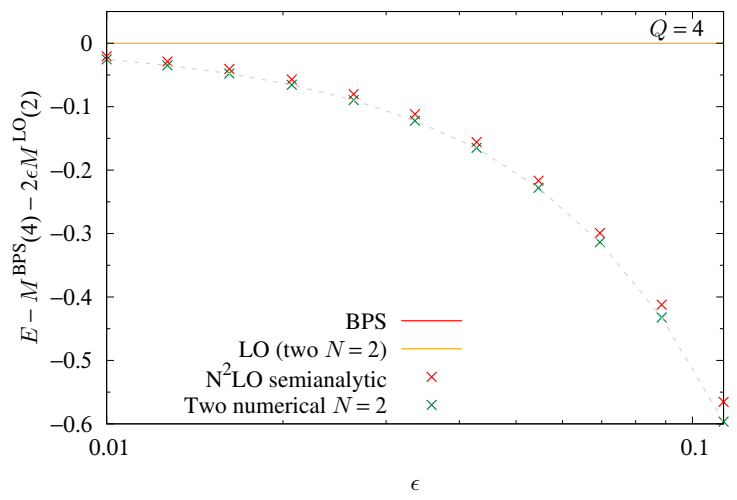

(b)

Figure 16. The energy (a) and $\mathrm{N}^{2} \mathrm{LO}$ perturbation energy of the lowest energy solutions in the $Q=4$ homotopy class, which is made of two $N=2$ compactons with separation distance $2 a=0$. The total energy (a) shows the BPS energy (red), the LO correction from the kinetic term of order $\epsilon$ (orange), the full $\mathrm{N}^{2} \mathrm{LO}$ perturbation energy obtained by PDE calculations (red crosses) and finally in comparison with the full PDE calculations of the solutions without perturbation theory (green crosses and connected with gray-dashed lines), all as functions of $\epsilon$. (b) shows the $\mathrm{N}^{2} \mathrm{LO}$ part of the energy only, so as to better see the accuracy of the comparison between the full numerical PDE energies (green crosses) and the $\mathrm{N}^{2} \mathrm{LO}$ perturbation energies (red crosses) as functions of $\epsilon$.

two $N=1$ solitons with opposite orientation as the background solution. Our guess is confirmed by the numerical simulation in figure 7 .

Similarly to the case of two $N=2$ compactons side-by-side, figures 17 and 18 which are for two $N=1$ compactons side-by-side, show the left-hand side of the composite soliton configuration with the gluing condition (5.9) imposed on the right-hand boundary of each panel for $\epsilon=0.01$ and $\epsilon=0.0428$, respectively. The other edges of the panels are not the limit of the calculations, which have merely been cropped so as to render the content as clear as possible. The two figures 17 and 18 again display the 6 columns: the perturbations $\delta f$ and $\delta \theta$ as well as the three components of the vector perturbations $\delta \phi$, and finally the perturbation energy density at $\mathrm{N}^{2} \mathrm{LO}$. Similarly to the $N=2$ case, the largest $\theta$-dependence is induced for very small separation distances $2 a$ and especially for $a=0$. It is clearly visible from the third and fourth columns of the figures that the compacton background solutions are $N=1$ baby Skyrmions, where cusp condition only changes sign twice around the perimeter of the compacton. As before, the width of the perturbation due to the cusp condition grows with $\epsilon$ and hence is more pronounced in figure 18 than in figure 17. For both values of $\epsilon$, the effect of the presence of the other compacton has almost disappeared at the separation distance $2 a=2 \times 0.256$. In the third component of the vector perturbations $\delta \phi^{3}$, we can see a dipolar structure emerging in the fifth columns of figures 13 and 14, which could be interpreted as the binding force between the two $N=2$ compactons pulling in the constituent baby Skyrmions inside the $N=2$ compacton. For larger values of $\epsilon$, this is eventually the effect that stretches the baby Skyrmions in the $Q=4$ solution made of two (deformed) $N=2$ baby Skyrmions side-by-side, see figure 4 . 

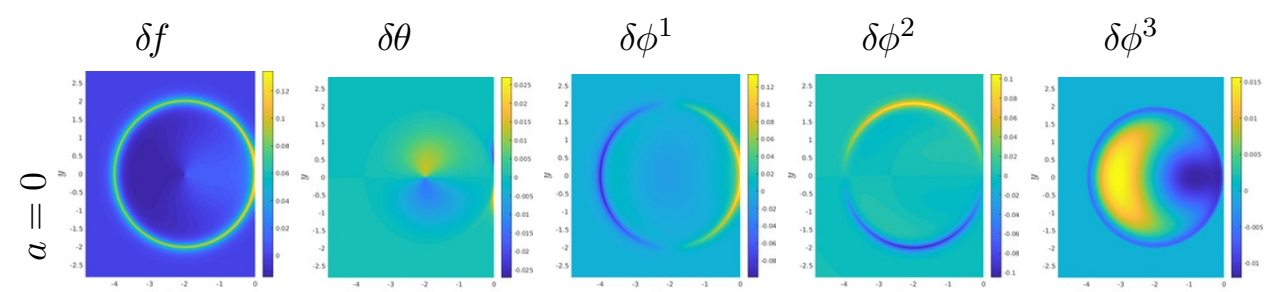

$\mathcal{E}^{\text {perturb }}$
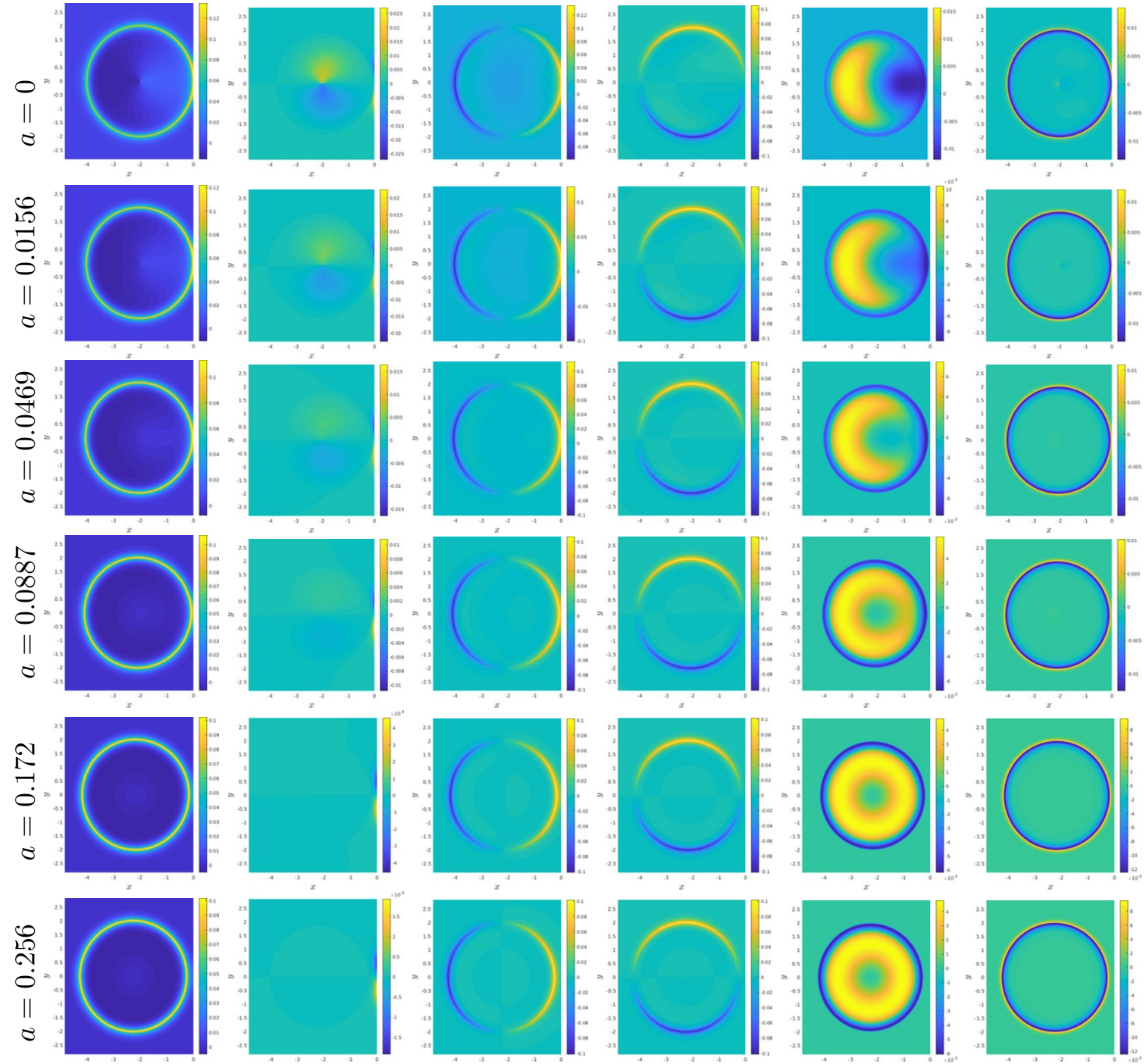

Figure 17. Numerical solution for $\delta f$ and $\delta \theta$ for two $N=1$ compactons situated next two each other with separation distance $2 a$ for $\epsilon=0.01$. Due to symmetry of the problem, only the left-hand side is calculated and shown. The gluing conditions (5.9) are imposed at $x=0$, which is located at the right-most part of every panel. Each row displays the perturbations $\delta f, \delta \theta, \delta \phi^{1}, \delta \phi^{2}, \delta \phi^{3}$ and the energy density of the perturbation $\mathcal{E}^{\text {perturb }}$, each in its column. Each panel is cropped so as to render the content as clear as possible; the left, top and bottom border do not represent the size of the simulations. 

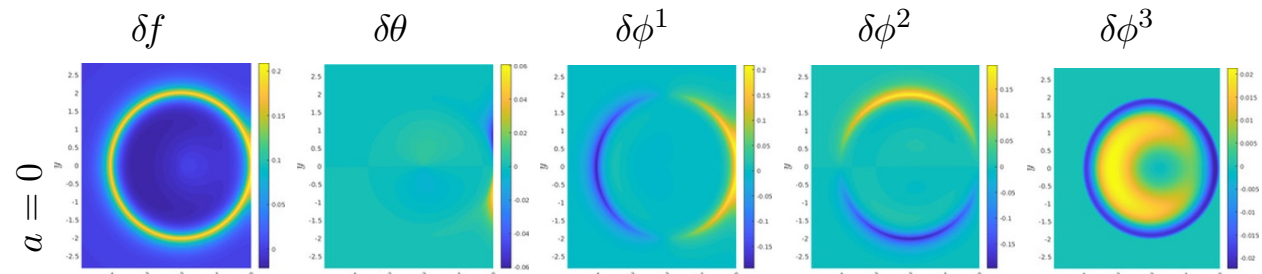

$\mathcal{E}^{\text {perturb }}$
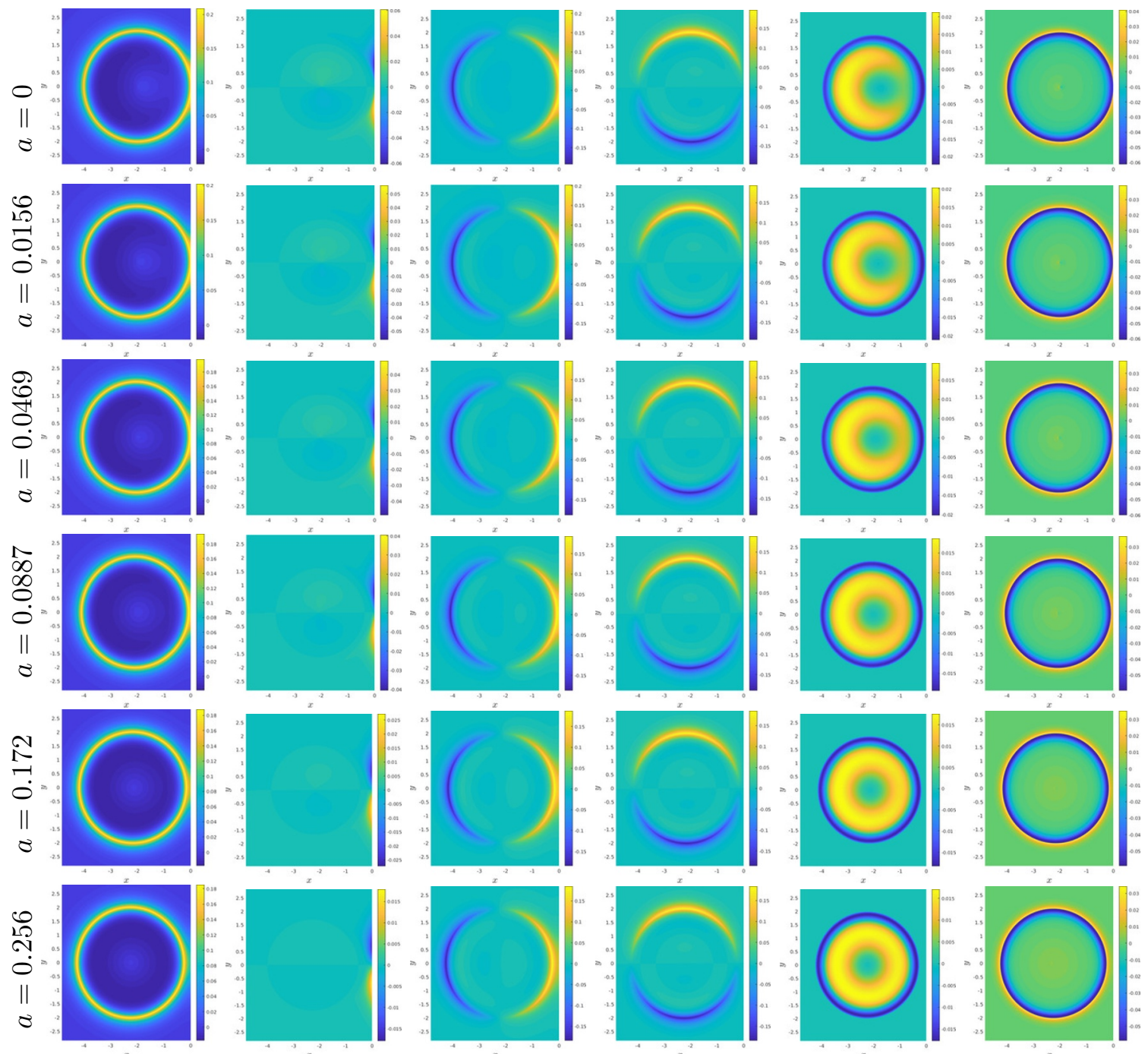

Figure 18. Numerical solution for $\delta f$ and $\delta \theta$ for two $N=1$ compactons situated next two each other with separation distance $2 a$ for $\epsilon=0.0428$. Due to symmetry of the problem, only the lefthand side is calculated and shown. The gluing conditions (5.9) are imposed at $x=0$, which is located at the right-most part of every panel. Each row displays the perturbations $\delta f, \delta \theta, \delta \phi^{1}, \delta \phi^{2}$, $\delta \phi^{3}$ and the energy density of the perturbation $\mathcal{E}^{\text {perturb }}$, each in its column. Each panel is cropped so as to render the content as clear as possible; the left, top and bottom border do not represent the size of the simulations. 


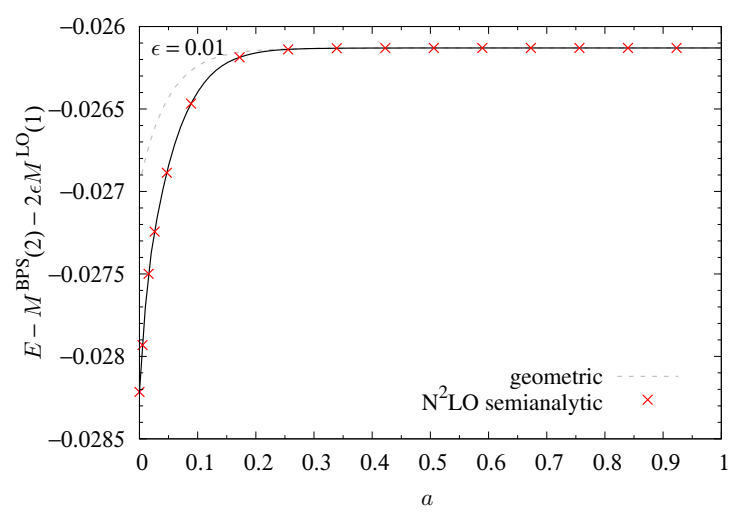

(a)

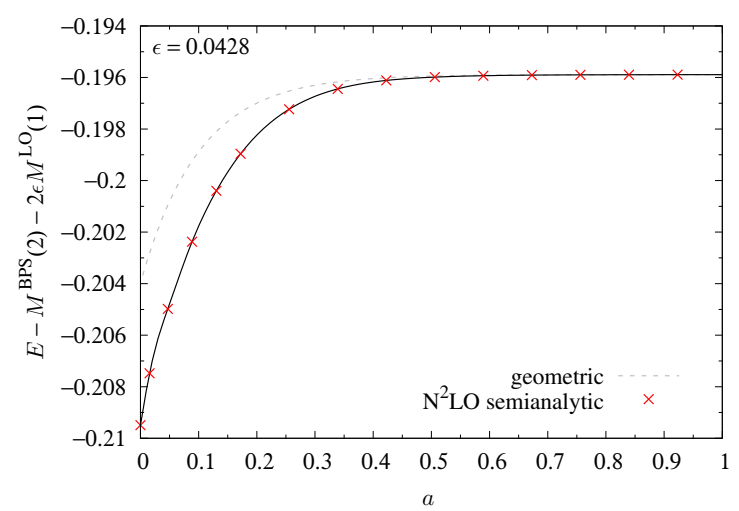

(c)

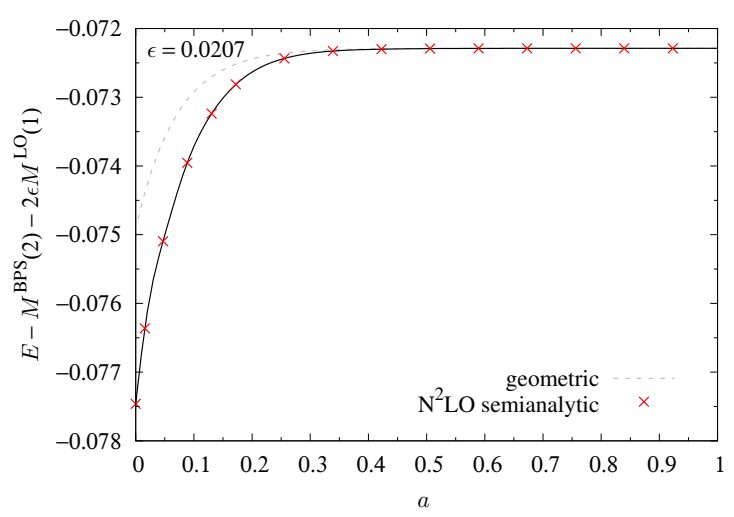

(b)

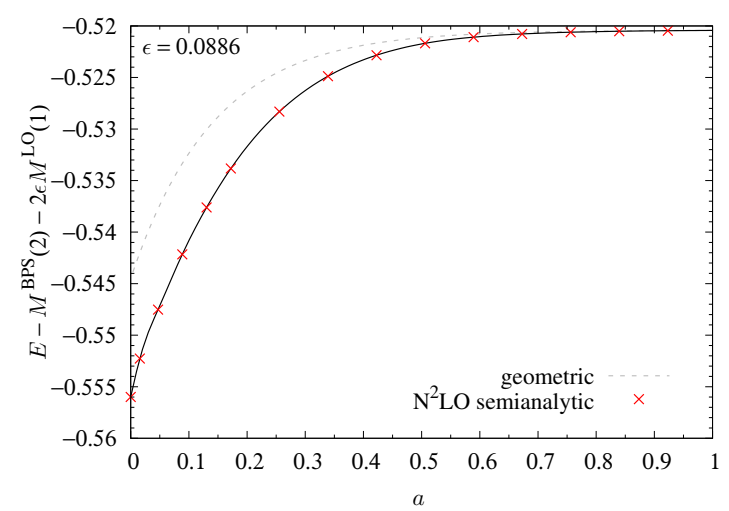

(d)

Figure 19. The $\mathrm{N}^{2} \mathrm{LO}$ corrections to the energy for two $N=1$ baby Skyrmions as a function of the separation distance $2 a$, see figure 12. The panels correspond to different values of $\epsilon$ : (a) $\epsilon=0.01$, (b) $\epsilon=0.0207$, (c) $\epsilon=0.0428$ and (d) $\epsilon=0.0886$. The gray-dashed line is calculated as the geometric cut at $x=0$ of the spherically symmetric compacton energy. The red crosses show the results of the $\mathrm{N}^{2} \mathrm{LO}$ contribution to the energy from the PDE calculation of $\delta f$ and $\delta \theta$. The black line is a cubic interpolation between the PDE results.

The effect is clearly absent in figures 17 and 18, where the compactons are $N=1$ baby Skyrmions and hence cannot be split up (viz. fission) into smaller parts.

Figure 19 shows the perturbative contribution to the energy at $\mathrm{N}^{2} \mathrm{LO}$ for two $N=1$ baby Skyrmions as function of the separation distance 2a, see figure 12. The four panels show $\epsilon=0.01, \epsilon=0.0207, \epsilon=0.0428$ and $\epsilon=0.0886$, respectively and for all panels, the smallest energy (meaning the largest negative contribution to the energy) is at $a=0$, which means when the two compactons exactly touch each other at a point, analogously to the two $N=2$ baby Skyrmion case. A difference with respect to the latter case is that for two $N=1$ baby Skyrmions, the $\mathrm{N}^{2} \mathrm{LO}$ contribution to the energy is always below the geometric energy, which is simply a geometric cut of the $\mathrm{N}^{2} \mathrm{LO}$ energy of the axially symmetric compacton at $x=0$. In particular, there is no crossing of the $\mathrm{N}^{2} \mathrm{LO}$ result and the geometric line, even for $\epsilon=0.0886$. This is probably because the gluing condition is 


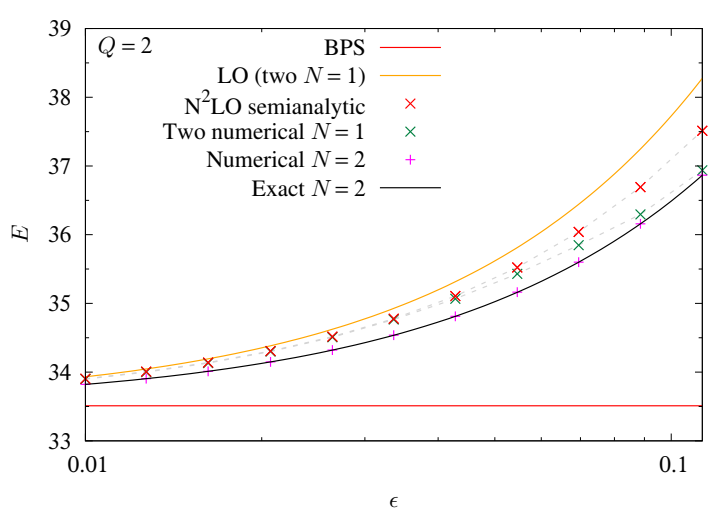

(a)

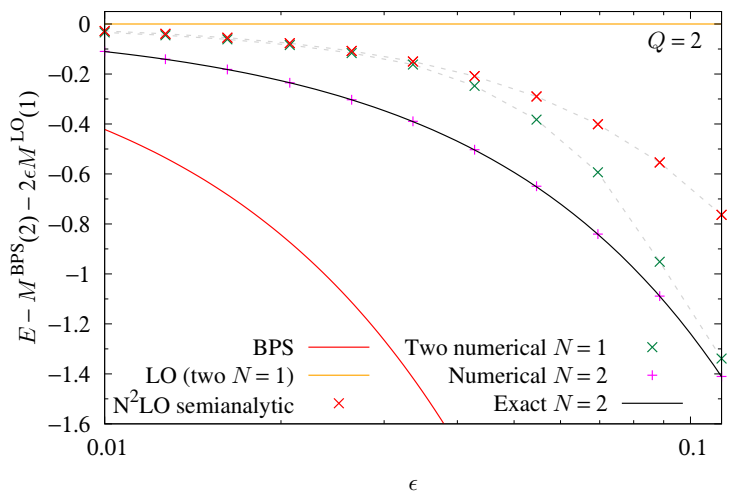

(b)

Figure 20. The energy (a) and $\mathrm{N}^{2} \mathrm{LO}$ perturbation energy of the metastable solution in the $Q=2$ homotopy class, which is made of two $N=1$ compactons with separation distance $2 a=0$. The total energy (a) shows the BPS energy (red), the LO correction from the kinetic term of order $\epsilon$ (orange), the full $\mathrm{N}^{2} \mathrm{LO}$ perturbation energy obtained by PDE calculations (red crosses) in comparison with the full numerical PDE calculations of the solutions without perturbation theory (green crosses), and for reference the full numerical PDE solutions of the axially symmetric $N=2$ baby Skyrmions (magenta pluses) as well as the exact $N=2$ baby Skyrmions obtained by ODEs, all as functions of $\epsilon$. (b) shows the $\mathrm{N}^{2} \mathrm{LO}$ part of the energy only, so as to better see the accuracy of the comparison between the full numerical PDE energies (green crosses) and the $\mathrm{N}^{2} \mathrm{LO}$ perturbative energies (red crosses) as functions of $\epsilon$.

more relaxed for the $N=1$ compactons, since the winding of the background solution is less (i.e. minimal). In particular, this eases the gluing condition (5.7) which is more strict for the $N=2$ compacton's $\delta \phi^{2}$ component.

We will now compare the $\mathrm{N}^{2} \mathrm{LO}$ result of the perturbative contribution to the energy of the two $N=1$ baby Skyrmions sitting side-by-side (taking into account the binding energy between them) with the full numerical (brute-force) PDE calculation in figure 20. The result of the $\mathrm{N}^{2} \mathrm{LO}$ perturbation theory is shown with red crosses in the figure and should be compared with the green crosses for the full numerical PDE calculations. The two $N=1$ baby Skyrmions sitting side-by-side are only metastable and in fact the most stable result in the $Q=2$ sector is the single $N=2$ compacton, for which we do not need to calculate any binding energy. Hence this calculation is simply a test of the perturbative scheme on the two $N=1$ baby Skyrmions sitting side-by-side, even though they are only metastable. For large $\epsilon \gtrsim 0.15$ we know from the full numerical PDE computations of section 4 that the two $N=1$ baby Skyrmions side-by-side become unstable and merge into an axially symmetric $N=2$ baby Skyrmion. This fact is known already from the leading order (LO) result of the perturbation theory, see eq. (3.6). We can thus anticipate that the $\mathrm{N}^{2} \mathrm{LO}$ perturbative scheme will become inaccurate for large $\epsilon$, since the instability is due to the LO result and, in principle, not known to the NLO and $\mathrm{N}^{2} \mathrm{LO}$ computations. For this reason, for reference, we show also the single $N=2$ baby Skyrmion (with axial symmetry) in figure 20 as magenta pluses (full PDE results) and a black solid line (ODE results). 
From figure 20(b) we can see that the perturbative result for the $\mathrm{N}^{2} \mathrm{LO}$ contribution to the energy works impressively well for $\epsilon \lesssim 0.033$ but then deviates and smoothly tends to the curve for the single axially symmetric $N=2$ solution (black solid line).

We have now confirmed by comparison with full numerical PDE computations, that our perturbative scheme at $\mathrm{N}^{2} \mathrm{LO}$ works very well for the two $N=2$ baby Skyrmions sitting side-by-side at least for $\epsilon \lesssim 0.1$ and also for the two $N=1$ baby Skyrmions side-by-side for $\epsilon \lesssim 0.033$ (due to the instability of this solution). Since we already have a quite precise perturbative formula for the baby Skyrmion energy for small $N$ and small $\epsilon$ at $\mathrm{N}^{2} \mathrm{LO}$, see eq. (3.91), it will henceforth be worthwhile to separate out the binding energy of a "single bond" between two baby Skyrmions at $\mathrm{N}^{2} \mathrm{LO}$. This is straightforwardly extracted from the data used in figures 16 and 20. The result for two baby Skyrmions with total charge $2 N$ sitting side-by-side is hence given by

$$
\begin{aligned}
E^{\text {side-by-side }}(\epsilon, N \oplus N)= & 2 M^{\mathrm{BPS}}(N)+2 \epsilon M^{L O}(N)+2 \epsilon^{2} M^{\mathrm{NLO}}(N)+2 \epsilon^{3} M^{\mathrm{N}^{2} \mathrm{LO}}(N) \\
& +2 \epsilon^{4} M^{\text {residual }, 4}(N)+E^{\text {binding }}(\epsilon, N),
\end{aligned}
$$

where the first five terms are given by eq. (3.91) and the binding energy of a single bond is given by

$$
E^{\text {binding }}(\epsilon, N)= \begin{cases}-8.112 \times 10^{-4}-15.65 \epsilon^{2}+323.8 \epsilon^{3}-2882 \epsilon^{4}, & N=1, \\ -5.108 \times 10^{-4}-8.376 \epsilon^{2}+167.8 \epsilon^{3}-1097 \epsilon^{4}, & N=2,\end{cases}
$$

where we have not extracted an $N$-behavior, since for $N=1$ and $N=2$ data only we cannot determine whether the is a quadratic or a linear behavior (in $N$ ) in the coefficients. Obviously, the constant term in both of the above expressions is expected to vanish. We have, nevertheless, included it because forcing it to be zero significantly worsens the fit. At this stage it is not clear whether the nonvanishing constant term is numerical error or there is a contact term in the binding energy, although we did not predict such a term.

Figure 21 shows the binding energy extracted from the $\mathrm{N}^{2} \mathrm{LO}$ computations of the two baby Skyrmions side-by-side, shown in figures 16 and 20 for $N=2$ and $N=1$, respectively. For reference, we show also the binding energies at NLO. The fits of eq. (5.16) are displayed with black solid lines and are fitted to the region $\epsilon<0.05$. Figure 21(a) shows the actual binding energy in the baby Skyrme units, which should be compared to the energy of the baby Skyrmions in baby Skyrme units, see figure 2. In order to aid the comparison, we show the binding energy per baby Skyrmion in units of the baby-Skyrmion mass in figure 21(b).

\section{Composite solutions}

One could now in principle construct "nuclei" of any $Q$ by rotating the constituent compactons such that the colors are matching at the "bond" and to a first approximation, the total energy can be calculated from eq. (5.16) with the addition of $E^{\text {binding }}(\epsilon, N)$ for each "bond" binding the nucleus. The near-BPS property allows one to tune $\epsilon$ until realistic binding energies are obtained. Of course, this only provides one with the ground state 


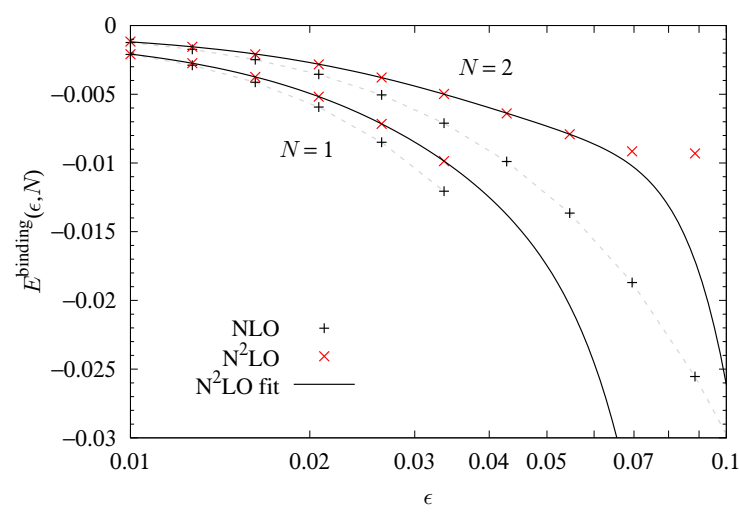

(a)

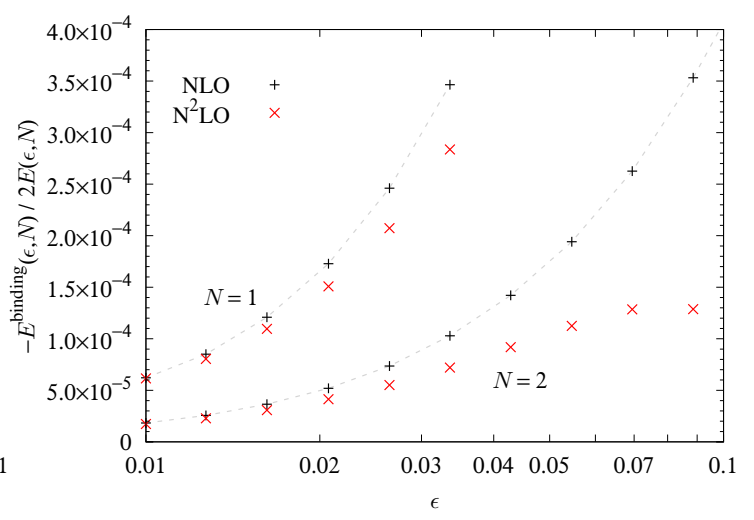

(b)

Figure 21. (a) Binding energy as a function of $\epsilon$ in form of a single "bond" between two $N=1,2$ baby Skyrmions. The red crosses are the $\mathrm{N}^{2} \mathrm{LO}$ results, while the black pluses are the NLO results shown for reference. For $N=1$ the data is truncated at $\epsilon \sim 0.033$ since the two composite $N=1$ baby Skyrmions side-by-side become unstable and will merge. The black solid lines are the fits given in eq. (5.16). (b) Binding energy between the two charge- $N$ baby Skyrmions divided by the total energy of the two charge- $N$ baby Skyrmions.

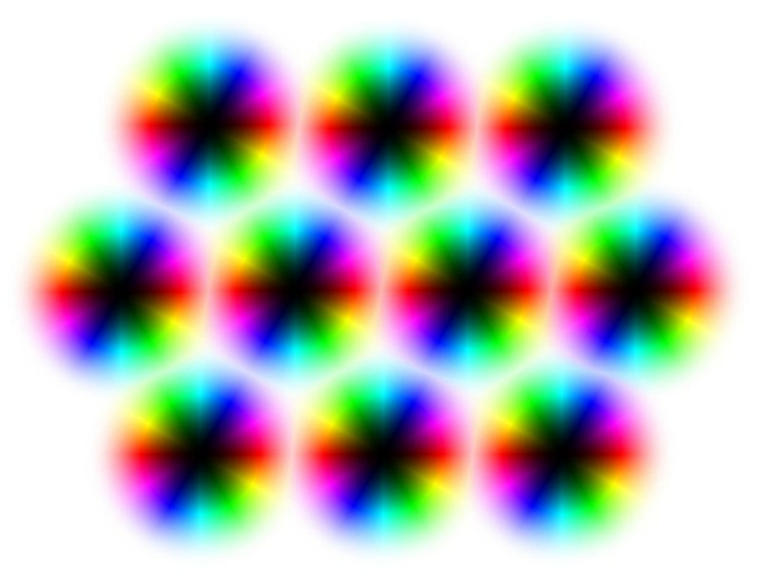

Figure 22. A sketch of a possible lattice for nuclear matter in the baby-Skyrme model. Each constituent baby Skyrmion is placed such that the colors match between the latter and the neighboring baby Skyrmion.

energies and further development is needed for calculating the excitational spectrum. And of course, the baby Skyrme model is just a toy model.

We could further contemplate how the limit of large nuclei or nuclear matter would look like. In figure 22 we show a possibility for an infinite "crystal" lattice of which large nuclei could be cut out from or nuclear matter could be made of. This particular lattice could be continued indefinitely. Nevertheless the shown lattice is made of ten baby Skyrmions and the energy to a first approximation could be calculated for any small value of $\epsilon$ by taking into account 19 bonds in the formula (5.16). 
Or course, the lattice phase would be determined by minimizing the energy and it could be for some $\epsilon$ that the square lattice is energetically preferred to this triangle lattice, displayed in figure 22. Nevertheless, it is expected that for sufficiently large $\epsilon$, the large- $Q$ solution becomes a chain [34] and the lattice would then only exist at finite density.

\section{Conclusion and discussion}

In this paper, we have studied the near-BPS regime of the baby Skyrme model with the standard pion mass term as the potential and the kinetic term as the BPS-breaking perturbation with coefficient $\epsilon$. The BPS solutions attain the BPS bound and hence the largest contribution to the energy is the BPS mass. The leading order contribution comes from the kinetic term and is of order $\epsilon$. To this order, there is no contribution to the binding energy and no forces between the compactons. Nevertheless, we find from the leading-order result that the $N=2$ baby Skyrmion is the stable solution for small $\epsilon$. In order to get a nonvanishing tail outside the compactons, we must go to third order in $\epsilon$ and not truncate the solution to a finite order in $\epsilon$, that is, the solution is of the form $\exp (-m r / \sqrt{\epsilon})$, which in some sense is an all-order solution in $\epsilon$. We test our perturbative scheme on the axially symmetric compacton solutions and calculate the $\mathrm{N}^{2} \mathrm{LO}$ corrections to the energy obtaining impressively good results. A key to making the perturbation around the compactons capture the physics of the baby Skyrmion is a delicate cusp condition that must be imposed on the compacton boundary. We then turn to a large-scale brute-force computation of the full PDEs and obtain numerical solutions for the baby Skyrmions for values of $\epsilon$ in the range $[0.01,1]$ and clearly observe that the baby Skyrmions tend to almost unperturbed compactons that touch each other at a single point in the attractive channel. We also find a new solution, by studying the $N=4$ axially symmetric baby Skyrmion which for $\epsilon \simeq 0.15$ collapses to a new metastable solution composed of four $N=1$ solutions in a tetrahedral arrangement - this solution is to the best of our knowledge new. The surprise about this solution is that the neighboring $N=1$ solutions should merge into an $N=2$ solution, but this does not happen for the tetrahedral solution, probably because of the discrete symmetry yielding a delicate balance of the solution. We also study the two $N=1$ baby Skyrmions side-by-side and find they are metastable for small values of $\epsilon \lesssim 0.15$. Finally, we turn to calculating the binding energies of two $N=1,2$ baby Skyrmions sitting side-by-side using our perturbative scheme. This is possible by imposing a gluing condition on the perturbation field and the results are in very good agreement with the brute-force numerical computations for small values of $\epsilon \lesssim 0.1$. Finally, we fit the results we have obtained, yielding an energy formula as a function of $\epsilon$ and $N$ and the binding energy for each bond is fitted for the cases of $N=1$ and $N=2$.

In this paper, we considered the case of the pion mass term as the potential (2.5). For this potential, in the near-BPS limit, the mass of the "pions" (the perturbative particles) goes like $\frac{1}{\sqrt{\epsilon}}$ due to the $\epsilon$ in front of the Dirichlet (kinetic) term; thus at a sufficiently small $\epsilon$ they become even more massive than the baby Skyrmion itself. Clearly, in QCD terms, this would be phenomenologically unacceptable. There are other near-BPS limits that resolve this problem. For example, by choosing a different type of potential for the main BPS part of the model, for which the contribution to the pion mass vanishes, e.g. that of 
eq. (2.4) with $p$ bigger than one. One could then add the massive term in the perturbation together with the kinetic term (so that it also is of order $\epsilon$ in the Lagrangian). These types of near-BPS limits have been discussed in the Skyrme literature, see for example refs. $[9,26,36,37]$. The main new observation we wish to make here is the following. We showed that the binding energy for the compacton case goes like $\epsilon^{2}$ and not as $\epsilon$ as would be expected from the restricted harmonic argument. Thus a considerably small binding energy could possibly be obtained without pushing the pion mass too high.

Both the analytical guess and the numerical type of work and in particular the semianalytic numerics we performed in the present paper are tailored to the compacton case, so the non-compacton case will require a different approach or at least crucial modifications. So far we know of several types of near-BPS solitonic models which can produce small classical binding energies. There are big differences among them, and it is not clear which one is most suitable for phenomenological applications to nuclear physics. For example there are cases in which the small binding energy is achieved by nuclei with size much smaller than the separation, and a relative position fixed by a potential which can be computed in the linear approximation [9, 38], i.e. quite different from the type of bound state studied in the present paper. Sometimes it is possible to interpolate between these two regimes by dialing a parameter, for example in holographic QCD with the 't Hooft coupling [39]. It may be that by studying different types of potentials of the baby Skyrme model even more diverse near-BPS behaviors will be discovered.

A crucial point of the perturbative scheme deployed in this paper is that the leading order (LO) energy is finite. In appendix A, we have extended the BPS solutions and the leading-order mass correction to the case of various generalized potentials. For the straightforward generalization of the pion mass term, which is simply the latter to the power $s$ (i.e. $\left.V \sim\left(1-\phi^{3}\right)^{s}\right)$, the BPS solution yields a finite integral for the kinetic energy for all the values of $s$ studied in the appendix. Changing the potential to the modified pion mass, which possesses domain walls, (i.e. $V \sim\left(1-\left(\phi^{3}\right)^{2}\right)^{s}$, the BPS solutions can be found only indirectly, except for $s=1$. But yet worse, for the solution with $s=1$, the leading order mass correction diverges. This demonstrates that the problem of perturbation around the compacton is not due to the cusp, that one might naively expect, but is due to the behavior of the BPS solution at the origin (i.e. at $r=0$ ). For the BPS Skyrme model in $3+1$ dimensions, a similar issue with the leading-order mass correction is known, for example for the solutions in ref. [1]. As illustrated in the appendix A, it may well be that one must find an appropriate class of potentials for the BPS Skyrme model, in order to be able to port the perturbative scheme to the $3+1$ dimensional model.

\section{Acknowledgments}

We thank Calum Ross, Martin Speight, Andrzej Wereszczynski and Wojtek Zakrzewski for useful discussions. The work of M.B. and S.B. is supported by the INFN special project grant "GAST (Gauge and String Theory)". S.B.G. thanks the Outstanding Talent Program of Henan University for partial support. The work of S.B.G. is supported by the National Natural Science Foundation of China (Grant No. 11675223 and 12071111). 


\section{A The leading order energy for various potentials}

In this appendix, will extend the leading order calculation, in $\epsilon$, of the energy of the baby Skyrmion to the cases of different potentials than the traditional mass term.

Although the leading order (LO) contribution to the energy comes from the kinetic term, it depends on the potential via the solution which depends strongly on the choice of potential. The BPS equation (2.16) for a generic potential takes the form

$$
\frac{\partial_{r} \zeta}{r}= \pm \frac{\left(1+\zeta^{2}\right)^{2}}{2 \sqrt{2} N \zeta} \sqrt{V(\zeta)}
$$

We will consider a few cases in turn in the next subsections.

\section{A.1 $\left(1-\phi^{3}\right)^{s}$}

Starting with the simplest generalization of the standard pion mass term, we consider the latter to the power $s$ :

$$
\begin{aligned}
V & =\frac{m^{2}}{s}\left(1-\phi^{3}\right)^{s} \\
& =\frac{m^{2}}{s}\left(\frac{2 \zeta^{2}}{1+\zeta^{2}}\right)^{s}
\end{aligned}
$$

which yields the BPS equation

$$
\frac{\partial_{r} \zeta}{r}= \pm \frac{2^{\frac{s-3}{2}} m}{N \sqrt{s}}\left(1+\zeta^{2}\right)^{2-\frac{s}{2}} \zeta^{s-1}
$$

which we can integrate as

$$
\int \frac{\mathrm{d} \zeta}{\zeta^{s-1}\left(1+\zeta^{2}\right)^{2-\frac{s}{2}}}= \pm \frac{2^{\frac{s-5}{2}} m}{N \sqrt{s}} r^{2} \mp \frac{\xi_{0}^{2}}{2-s}, \quad 0<s<2 .
$$

Defining the compacton radius

$$
R \equiv \sqrt{\frac{2^{\frac{5-s}{2}} \sqrt{s} N}{m}}, \quad 0<s<2,
$$

we can write the equation with the lower sign as

$$
\left(\frac{\zeta}{\sqrt{1+\zeta^{2}}}\right)^{2-s}=\xi_{0}^{2}-\xi^{2}, \quad 0<s<2,
$$

which can be inverted to give the solution

$$
\zeta=\frac{\left(\xi_{0}^{2}-\xi^{2}\right)^{\frac{1}{2-s}}}{\sqrt{1-\left(\xi_{0}^{2}-\xi^{2}\right)^{\frac{2}{2-s}}}}, \quad 0<s<2 .
$$

Finally, we move the singularity to $\xi=0$ by setting $\xi_{0}=1$ :

$$
\zeta=\frac{\left(1-\xi^{2}\right)^{\frac{1}{2-s}}}{\sqrt{1-\left(1-\xi^{2}\right)^{\frac{2}{2-s}}}}, \quad 0<s<2 .
$$




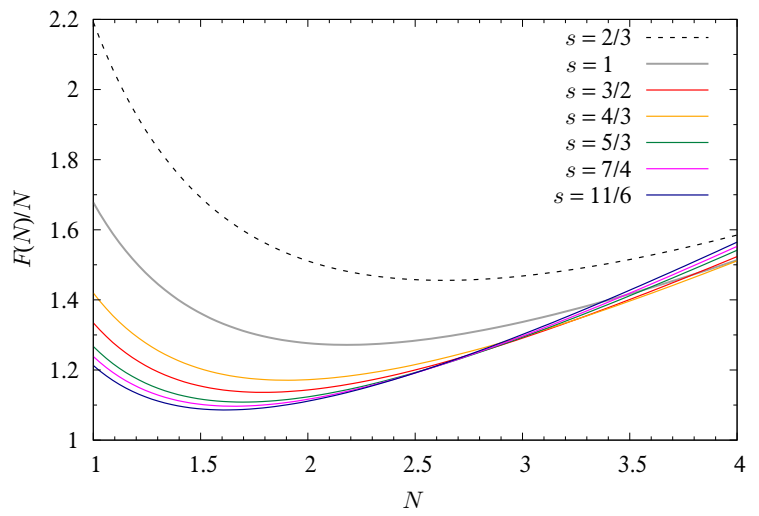

Figure 23. The leading-order mass correction function $F(N)=M^{\mathrm{LO}}(N) / 4 \pi$ as function of $N$ for various values of $s$ of the potential (A.2). Clearly, in all cases, the energy per $N$ is smaller for $N=2$ than for $N=1$.

The BPS bound is still attained, but now we can calculate the LO contribution to the energy using the above solution

$$
\begin{aligned}
\epsilon M^{\mathrm{LO}}(N) & =4 \pi \epsilon \int_{0}^{1} \mathrm{~d} \xi \frac{\xi \zeta_{\xi}^{2}+\frac{N^{2}}{\xi} \zeta^{2}}{\left(1+\zeta^{2}\right)^{2}} \\
& =-4 \pi \epsilon \int_{0}^{1} \mathrm{~d} \xi \frac{\left(1-\zeta^{2}\right)^{\frac{4}{s-2}}\left[4 \zeta^{4}\left(1-\zeta^{2}\right)^{-2-\frac{4}{s-2}}+N^{2}(2-s)^{2}\left(1-\left(1-\zeta^{2}\right)^{\frac{2}{s-2}}\right)^{2}\right]}{(s-2)^{2} \zeta\left(1-\left(1-\zeta^{2}\right)^{\frac{2}{s-2}}\right)}
\end{aligned}
$$

with $0<s<2$. Unfortunately, we do not know how to perform this integral for arbitrary values of $s$ in the given interval, but it can be carried out for certain specific values

$$
\begin{aligned}
\epsilon M^{\mathrm{LO}}(N) & =4 \pi \epsilon F(N) \\
F(N) & = \begin{cases}\left(\log 2-\frac{5}{12}\right) N^{2}+\frac{1}{8}(\sqrt{3} \pi+9 \log 3), & (\mathrm{A} .10) \\
\frac{7}{24} N^{2}+2 \log 2, & s=\frac{2}{3}, \\
\frac{533 N^{2}}{1680}-\pi+6 \log 2, & s=1, \\
\frac{37 N^{2}}{120}-\frac{1}{4} \sqrt{3} \pi+\frac{9}{4} \log 3, & s=\frac{3}{2}, \\
\frac{18107 N^{2}}{55440}-\frac{3}{2} \sqrt{3} \pi+\frac{9}{2} \log 3+6 \log 2, & s=\frac{4}{3}, \\
\frac{95549 N^{2}}{288288}-2(1+\sqrt{2}) \pi+4 \sqrt{2} \operatorname{arccoth}(\sqrt{2})+16 \log 2, & s=\frac{5}{3}, \\
\frac{3602044091 N^{2}}{10708457760}-3(2+\sqrt{3}) \pi+9 \log 12-\frac{3}{4} \sqrt{3} \log (18817-10864 \sqrt{3}), & s=\frac{7}{4}, \\
& s=\frac{11}{6} .\end{cases}
\end{aligned}
$$

Figure 23 shows the function $F(N)$ for various values of $s$.

Since the BPS mass does not contribute to the determination of $N_{\star}$, to leading order $(\mathrm{LO}), N_{\star}$ is determined by

$$
\frac{\mathrm{d}}{\mathrm{d} N}\left(\frac{E(\epsilon, N)}{N}\right)=\frac{\mathrm{d}}{\mathrm{d} N}\left(\frac{F(N)}{N}\right)=0
$$




\begin{tabular}{|c|llll|}
\hline$s$ & $N=1$ & $N=2$ & $N=3$ & $N=4$ \\
\hline $2 / 3$ & 2.193 & 1.511 & $1.468^{\star}$ & 1.585 \\
1 & 1.678 & $1.276^{\star}$ & 1.337 & 1.513 \\
$3 / 2$ & 1.335 & $1.143^{\star}$ & 1.291 & 1.523 \\
$4 / 3$ & 1.420 & $1.172^{\star}$ & 1.296 & 1.511 \\
$5 / 3$ & 1.267 & $1.123^{\star}$ & 1.293 & 1.542 \\
$7 / 4$ & 1.239 & $1.116^{\star}$ & 1.297 & 1.553 \\
$11 / 6$ & 1.213 & $1.111^{\star}$ & 1.301 & 1.565 \\
\hline
\end{tabular}

Table 1. The function $F(N) / N$ determining which solution has the smallest energy per $N$ as function of $N$ at LO. The smallest number is marked with a ${ }^{\star}$. Except for $s=\frac{2}{3}$ for which $N=3$ has the smallest energy at LO, all the cases have the smallest energy at LO for $N=2$.

yielding

$$
N_{\star}= \begin{cases}\sqrt{\frac{3(\sqrt{3} \pi+9 \log 3)}{24 \log 2-10}}, & s=\frac{2}{3}, \\ 4 \sqrt{\frac{3 \log 2}{7}}, & s=1, \\ 4 \sqrt{\frac{105}{533}(6 \log 2-\pi)}, & s=\frac{3}{2}, \\ \sqrt{\frac{30}{37}(9 \log 3-\sqrt{3} \pi)}, & s=\frac{4}{3}, \\ 6 \sqrt{\frac{2310}{18107}(\log 432-\sqrt{3} \pi)}, & s=\frac{5}{3}, \\ 12 \sqrt{\frac{2002}{95549}(-2(1+\sqrt{2}) \pi+4 \sqrt{2} \operatorname{arccoth}(\sqrt{2})+16 \log 2),} & s=\frac{7}{4}, \\ 6 \sqrt{\frac{223092870}{3602044091}(-4(2+\sqrt{2}) \pi+12 \log 12-\sqrt{3} \log (18817-10864 \sqrt{3}))}, & s=\frac{11}{6},\end{cases}
$$

or in approximate numbers

$$
N_{\star} \simeq \begin{cases}2.633, & s=\frac{2}{3}, \\ 2.180, & s=1 \\ 1.791, & s=\frac{3}{2}, \\ 1.899, & s=\frac{4}{3}, \\ 1.697, & s=\frac{5}{3}, \\ 1.654, & s=\frac{7}{4}, \\ 1.614, & s=\frac{11}{6} .\end{cases}
$$

When $N_{\star}$ is not close to an integer, it is less clear which $N$ actually provides the minimal energy at LO. Thus we provide the data for $F(N) / N$ in table 1 


\section{A.2 $\left(1-\left(\phi^{3}\right)^{2}\right)^{s}$}

A straightforward generalization of the so-called modified pion mass term can be written as

$$
\begin{aligned}
V & =\frac{m^{2}}{2 s}\left(1-\left(\phi^{3}\right)^{2}\right)^{s} \\
& =\frac{m^{2}}{2 s}\left(\frac{2 \zeta}{1+\zeta^{2}}\right)^{2 s}
\end{aligned}
$$

which yields the BPS equation

$$
\frac{\partial_{r} \zeta}{r}= \pm \frac{2^{s-2} m}{N \sqrt{s}}\left(1+\zeta^{2}\right)^{2-s} \zeta^{s-1}
$$

which we can integrate as

$$
\int \frac{\mathrm{d} \zeta}{\zeta^{s-1}\left(1+\zeta^{2}\right)^{2-s}}= \pm \frac{2^{s-3} m}{N \sqrt{s}} r^{2} \mp \xi_{0}^{2}, \quad 0<s<2 .
$$

Defining the compacton radius

$$
R \equiv \sqrt{\frac{2^{3-s} \sqrt{s} N}{m}},
$$

we can write the equation with the lower sign as

$$
\frac{\zeta^{2-s}}{2-s}{ }_{2} F_{1}\left[2-s, 1-\frac{s}{2} ; 2-\frac{s}{2} ;-\zeta^{2}\right]=\xi_{0}^{2}-\xi^{2}, \quad 0<s<2,
$$

where ${ }_{2} F_{1}$ is Gauss' hypergeometric function. Unfortunately, we do not know how to invert this equation to get a closed expression for $\zeta$, which makes it difficult to evaluate the LO energy analytically.

However, if we restrict to the case of $s=1$, we get the equation

$$
\arctan \zeta=\xi_{0}^{2}-\xi^{2}
$$

which can readily be inverted as

$$
\zeta=\tan \left(1-\xi^{2}\right)
$$

where we have set $\xi_{0}=1$. This solution, however, does not yield a finite result for the leading-order correction to the mass.

Open Access. This article is distributed under the terms of the Creative Commons Attribution License (CC-BY 4.0), which permits any use, distribution and reproduction in any medium, provided the original author(s) and source are credited.

\section{References}

[1] C. Adam, J. Sanchez-Guillen and A. Wereszczynski, A Skyrme-type proposal for baryonic matter, Phys. Lett. B 691 (2010) 105 [arXiv: 1001.4544] [InSPIRE].

[2] E. Bonenfant and L. Marleau, Nuclei as near BPS-Skyrmions, Phys. Rev. D 82 (2010) 054023 [arXiv: 1007.1396 ] [INSPIRE]. 
[3] C. Adam, J. Sanchez-Guillen and A. Wereszczynski, A BPS Skyrme model and baryons at large $N_{c}$, Phys. Rev. D 82 (2010) 085015 [arXiv: 1007.1567] [InSPIRE].

[4] E. Bonenfant, L. Harbour and L. Marleau, Near-BPS Skyrmions: non-shell configurations and Coulomb effects, Phys. Rev. D 85 (2012) 114045 [arXiv:1205.1414] [inSPIRE].

[5] C. Adam, C. Naya, J. Sanchez-Guillen and A. Wereszczynski, Bogomol'nyi-Prasad-Sommerfield Skyrme model and nuclear binding energies, Phys. Rev. Lett. 111 (2013) 232501 [arXiv: 1312. 2960] [INSPIRE].

[6] C. Adam, C. Naya, J. Sanchez-Guillen, R. Vazquez and A. Wereszczynski, BPS Skyrmions as neutron stars, Phys. Lett. B $\mathbf{7 4 2}$ (2015) 136 [arXiv:1407.3799] [INSPIRE].

[7] C. Adam, C. Naya, J. Sanchez-Guillen, R. Vazquez and A. Wereszczynski, Neutron stars in the Bogomol'nyi-Prasad-Sommerfield Skyrme model: mean-field limit versus full field theory, Phys. Rev. C 92 (2015) 025802 [arXiv:1503.03095] [INSPIRE].

[8] C. Adam, M. Haberichter and A. Wereszczynski, Skyrme models and nuclear matter equation of state, Phys. Rev. C 92 (2015) 055807 [arXiv: 1509.04795] [InSPIRE].

[9] M. Gillard, D. Harland and M. Speight, Skyrmions with low binding energies, Nucl. Phys. B 895 (2015) 272 [arXiv: 1501.05455] [INSPIRE].

[10] R.A. Leese, M. Peyrard and W.J. Zakrzewski, Soliton scatterings in some relativistic models in $(2+1)$-dimensions, Nonlinearity 3 (1990) 773 [INSPIRE].

[11] B.M.A.G. Piette, W.J. Zakrzewski, H.J.W. Mueller-Kirsten and D.H. Tchrakian, A modified Mottola-Wipf model with sphaleron and instanton fields, Phys. Lett. B 320 (1994) 294 [INSPIRE].

[12] B.M.A.G. Piette, B.J. Schroers and W.J. Zakrzewski, Multi-solitons in a two-dimensional Skyrme model, Z. Phys. C 65 (1995) 165 [hep-th/9406160] [InSPIRE].

[13] B.M.A.G. Piette, B.J. Schroers and W.J. Zakrzewski, Dynamics of baby Skyrmions, Nucl. Phys. B 439 (1995) 205 [hep-ph/9410256] [INSPIRE].

[14] T. Gisiger and M.B. Paranjape, Solitons in a baby Skyrme model with invariance under volume/area preserving diffeomorphisms, Phys. Rev. D 55 (1997) 7731 [hep-ph/9606328] [INSPIRE].

[15] C. Adam, P. Klimas, J. Sanchez-Guillen and A. Wereszczynski, Compact baby Skyrmions, Phys. Rev. D 80 (2009) 105013 [arXiv:0909.2505] [InSPIRE].

[16] C. Adam, T. Romanczukiewicz, J. Sanchez-Guillen and A. Wereszczynski, Investigation of restricted baby Skyrme models, Phys. Rev. D 81 (2010) 085007 [arXiv: 1002.0851] [INSPIRE].

[17] J.M. Speight, Compactons and semi-compactons in the extreme baby Skyrme model, J. Phys. A 43 (2010) 405201 [arXiv: 1006.3754] [INSPIRE].

[18] A. Fert, N. Reyren and V. Cros, Magnetic Skyrmions: advances in physics and potential applications, Nature Rev. Mater. 2 (2017) 17031.

[19] B. Barton-Singer, C. Ross and B.J. Schroers, Magnetic Skyrmions at critical coupling, Commun. Math. Phys. 375 (2020) 2259 [arXiv:1812.07268] [INSPIRE].

[20] B.J. Schroers, Gauged $\sigma$-models and magnetic Skyrmions, SciPost Phys. 7 (2019) 030 [arXiv: 1905.06285] [INSPIRE].

[21] C. Ross, N. Sakai and M. Nitta, Skyrmion interactions and lattices in solvable chiral magnets, arXiv:2003.07147 [INSPIRE]. 
[22] B. Zumino, Supersymmetry and Kähler manifolds, Phys. Lett. B 87 (1979) 203 [inSPIRE].

[23] E.A. Bergshoeff, R.I. Nepomechie and H.J. Schnitzer, Supersymmetric Skyrmions in four-dimensions, Nucl. Phys. B 249 (1985) 93 [INSPIRE].

[24] L. Freyhult, The supersymmetric extension of the Faddeev model, Nucl. Phys. B 681 (2004) 65 [hep-th/0310261] [INSPIRE].

[25] C. Adam, J.M. Queiruga, J. Sanchez-Guillen and A. Wereszczynski, $N=1$ supersymmetric extension of the baby Skyrme model, Phys. Rev. D 84 (2011) 025008 [arXiv:1105.1168] [INSPIRE].

[26] S. Bolognesi and W. Zakrzewski, Baby Skyrme model, near-BPS approximations and supersymmetric extensions, Phys. Rev. D 91 (2015) 045034 [arXiv:1407.3140] [InSPIRE].

[27] C. Adam, J.M. Queiruga, J. Sanchez-Guillen and A. Wereszczynski, Extended supersymmetry and BPS solutions in baby Skyrme models, JHEP 05 (2013) 108 [arXiv:1304.0774] [INSPIRE].

[28] M. Nitta and S. Sasaki, BPS states in supersymmetric chiral models with higher derivative terms, Phys. Rev. D 90 (2014) 105001 [arXiv: 1406.7647] [inSPIRE].

[29] M. Nitta and S. Sasaki, Classifying BPS states in supersymmetric gauge theories coupled to higher derivative chiral models, Phys. Rev. D 91 (2015) 125025 [arXiv:1504.08123] [INSPIRE].

[30] S.B. Gudnason, M. Nitta and S. Sasaki, A supersymmetric Skyrme model, JHEP 02 (2016) 074 [arXiv: 1512.07557] [INSPIRE].

[31] S.B. Gudnason, M. Nitta and S. Sasaki, Topological solitons in the supersymmetric Skyrme model, JHEP 01 (2017) 014 [arXiv:1608.03526] [INSPIRE].

[32] S. Bolognesi and P. Sutcliffe, The Sakai-Sugimoto soliton, JHEP 01 (2014) 078 [arXiv: 1309.1396] [INSPIRE].

[33] J.M. Speight, Near BPS Skyrmions and restricted harmonic maps, J. Geom. Phys. 92 (2015) 30 [arXiv: 1406. 0739] [INSPIRE].

[34] D. Foster, Baby Skyrmion chains, Nonlinearity 23 (2010) 465 [arXiv:0904.3846] [INSPIRE].

[35] S.B. Gudnason and J.M. Speight, Realistic classical binding energies in the $\omega$-Skyrme model, JHEP 07 (2020) 184 [arXiv: 2004 .12862] [INSPIRE].

[36] M.-O. Beaudoin and L. Marleau, Near-BPS Skyrmions: constant baryon density, Nucl. Phys. $B \mathbf{8 8 3}$ (2014) 328 [arXiv:1305.4944] [INSPIRE].

[37] S.B. Gudnason, Exploring the generalized loosely bound Skyrme model, Phys. Rev. D 98 (2018) 096018 [arXiv: 1805.10898] [INSPIRE].

[38] S. Baldino, S. Bolognesi, S.B. Gudnason and D. Koksal, Solitonic approach to holographic nuclear physics, Phys. Rev. D 96 (2017) 034008 [arXiv:1703.08695] [INSPIRE].

[39] L. Bartolini, S. Bolognesi and A. Proto, From the Sakai-Sugimoto model to the generalized Skyrme model, Phys. Rev. D 97 (2018) 014024 [arXiv:1711.03873] [InSPIRE]. 